\title{
Accurate and homogeneous abundance patterns in solar-type stars of the solar neighbourhood: a chemo-chronological analysis ${ }^{\star}$
}

\author{
R. da Silva ${ }^{1}$, G. F. Porto de Mello ${ }^{2}$, A. C. Milone ${ }^{1}$, L. da Silva ${ }^{3}$, L. S. Ribeiro ${ }^{1}$, and H. J. Rocha-Pinto ${ }^{2}$ \\ 1 INPE, Divisão de Astrofísica, Av. dos Astronautas, 1758 São José dos Campos, 12201-970, Brazil \\ e-mail: dasilvr2@gmail.com \\ 2 UFRJ, Observatório do Valongo, Ladeira do Pedro Antônio 43, 20080-090 Rio de Janeiro, Brazil \\ 3 Observatório Nacional, Rua Gal. José Cristino 77, 20921-400 São Cristovão, Rio de Janeiro, Brazil
}

Received 28 December 2011 / Accepted 22 April 2012

\section{ABSTRACT}

\begin{abstract}
Aims. We report the derivation of abundances of $\mathrm{C}, \mathrm{Na}, \mathrm{Mg}, \mathrm{Si}, \mathrm{Ca}, \mathrm{Sc}, \mathrm{Ti}, \mathrm{V}, \mathrm{Cr}, \mathrm{Mn}, \mathrm{Fe}, \mathrm{Co}, \mathrm{Ni}, \mathrm{Cu}, \mathrm{Zn}, \mathrm{Sr}, \mathrm{Y}, \mathrm{Zr}, \mathrm{Ba}, \mathrm{Ce}, \mathrm{Nd}$, and $\mathrm{Sm}$ in a sample of 25 solar-type stars of the solar neighbourhood, correlating the abundances with the stellar ages, kinematics, and orbital parameters.

Methods. The spectroscopic analysis, based on data of high resolution and high signal-to-noise ratio, was differential to the Sun and applied to atomic line equivalent widths supplemented by the spectral synthesis of $\mathrm{C}$ and $\mathrm{C}_{2}$ features. We also performed a statistical study by using the method of tree clustering analysis, searching for groups of stars sharing similar elemental abundance patterns. We derived the stellar parameters from various criteria, with average errors of $30 \mathrm{~K}, 0.13 \mathrm{dex}$, and 0.05 dex, respectively, for $T_{\text {eff }}$, $\log g$, and $[\mathrm{Fe} / \mathrm{H}]$. The average error of the $[\mathrm{X} / \mathrm{Fe}]$ abundance ratios is 0.06 dex. Ages were derived from theoretical HR diagrams and membership of the stars in known kinematical moving groups.

Results. We identified four stellar groups: one having, on average, over-solar abundances $(\langle[\mathrm{X} / \mathrm{H}]\rangle=+0.26$ dex $)$, another with undersolar abundances $(\langle[\mathrm{X} / \mathrm{H}]\rangle=-0.24 \mathrm{dex})$, and two with intermediate values $(\langle[\mathrm{X} / \mathrm{H}]\rangle=-0.06$ and $+0.06 \mathrm{dex})$ but with distinct chemical patterns. Stars sharing solar metallicity, age, and Galactic orbit possibly have non-solar abundance ratios, a possible effect either of chemical heterogeneity in their natal clouds or migration. A trend of $[\mathrm{Cu} / \mathrm{Fe}]$ with $[\mathrm{Ba} / \mathrm{Fe}]$ seems to exist, in agreement with previous claims in the literature, and maybe also of $[\mathrm{Sm} / \mathrm{Fe}]$ with $[\mathrm{Ba} / \mathrm{Fe}]$. No such correlation involving $\mathrm{C}, \mathrm{Na}, \mathrm{Mn}$, and $\mathrm{Zn}$ is observed. The $[\mathrm{X} / \mathrm{Fe}]$ ratios of various elements show significant correlations with age. $[\mathrm{Mg} / \mathrm{Fe}],[\mathrm{Sc} / \mathrm{Fe}]$, and $[\mathrm{Ti} / \mathrm{Fe}]$ increase with age. $[\mathrm{Mn} / \mathrm{Fe}]$ and $[\mathrm{Cu} / \mathrm{Fe}]$ display a more complex behaviour, first increasing towards younger stars up to the solar age, and then decreasing, a result we interpret as possibly related to time-varying yields of SN Ia and the weak s-process in massive stars. The steepest negative age relation is due to $[\mathrm{Ba} / \mathrm{Fe}]$, but only for stars younger than the Sun, and a similar though less significant behaviour is seen for $\mathrm{Zr}$, $\mathrm{Ce}$, and $\mathrm{Nd}$. $[\mathrm{Sr} / \mathrm{Fe}]$ and $[\mathrm{Y} / \mathrm{Fe}]$ show a linearly increasing trend towards younger stars. The $[\mathrm{Cu} / \mathrm{Ba}]$ and $[\mathrm{Sm} / \mathrm{Ba}]$ therefore decrease for younger stars. We found that $[\mathrm{Ba} / \mathrm{Mg}],[\mathrm{Ba} / \mathrm{Zn}]$, and $[\mathrm{Sr}, \mathrm{Y}, \mathrm{Ba} / \mathrm{Sm}]$ increase but only for stars younger than the Sun, whereas the [Sr/Mg], [Y/Mg], [Sr/Zn], and [Y/Zn] ratios increase linearly towards younger stars over the whole age range.
\end{abstract}

Key words. stars: solar-type - stars: fundamental parameters - stars: abundances

\section{Introduction}

The Galactic chemical and dynamical history can be well framed by a series of average "laws" (see e.g. Edvardsson et al. 1993; McWilliam 1997), namely: the age-metallicity relation (which in principle is accessible by more than one chemical element), the [element/element] abundance ratios, the stellar metallicity frequency distribution, the Galactic metallicity gradient, and the star formation history, besides their mutual relationships as a function of space and time. The success of the Galactic chemical evolution models is to be judged by their ability to reproduce these constraints (see Allen \& Porto de Mello 2011).

In recent years there has been growing recognition that, even though such average laws are meaningful and fundamental, there may be considerable underlying complexity in the real Galaxy that has gone at least partly unappreciated. In the present age of very large spectroscopic databases and precise

^ Based on observations collected at the Cerro Tololo Inter-American Observatory, Chile. abundances for numerous chemical elements, a successful model must harmonise stellar evolution inputs such as the initial mass function, the star-formation rate, and mass loss processes, connecting these to the specific properties of Galactic components, and a large diversity of spatial and temporal structures, differing timescales for stellar nucleosynthetic yields and their sensitivities to differing metallicities.

During the evolution of the Galactic disc, nucleosynthesis in successive generations of stars occurs together with dynamical interactions with the interstellar gas. The states of the Galaxy in past periods of its evolution are still preserved in the abundance distributions of solar-type stars, which constitute an ideal population to study the chemical evolution. These stars have an age dispersion comparable to the age of the Galaxy. They are similar to the Sun in many physical parameters, allowing the application of a differential analysis and the consequent minimisation of theoretical shortcomings of atmospheric models and systematic errors. In addition, their chemical composition does not change in consequence of the mixing processes in their surfaces, 
which means that the present abundance of a given element is the same as in the time of their formation (an exception are the abundances of $\mathrm{Li}, \mathrm{Be}$, and $\mathrm{B}$, but these elements are not considered in this work). Therefore, the chemical abundance of solar-type stars, combined with kinematical, orbital, and evolutionary parameters (mass and age), provide a powerful tool to investigate the chemical and dynamical evolution of the Galaxy.

Over the last decade, several works have analysed the composition of disc dwarf stars of spectral types $F$ and $G$ (Chen et al. 2000; Reddy et al. 2003; Bensby et al. 2005; Chen et al. 2008; Neves et al. 2009). The metallicity ranges have become wider, the number of stars and elements studied has become larger, stars with and without detected planets have been compared, and the chemical distinction between thin and thick stellar population has been refined. Here we specifically ask, when regarded in as high a level of detail as possible with present techniques, to what extent the relative abundances of chemical elements can be traced as a function of age, the nature of underlying nucleosynthetic processes, and whether these properties can be statistically grouped for the nearby solar-type stars, defining "snapshots" relevant to the chemo-chronological evolution of the Galaxy.

In this work we present a multi-elemental spectroscopic analysis of a sample of 25 solar-type stars in the solar neighbourhood, all members of the thin disc stellar population (excepting one star in the transition between thin and thick discs). We have performed the determination of atmospheric parameters (effective temperature, metallicity, surface gravity, and microturbulence velocity), mass, age, kinematical and orbital parameters, and elemental abundances based on equivalent widths or spectral synthesis. Three different criteria were used to pin down the stellar effective temperatures, and they showed excellent internal consistency. We have also performed a statistical study of our abundance results using the method of tree clustering analysis (Everitt et al. 2001), through which we looked for stellar groups that share similar abundances in $[\mathrm{X} / \mathrm{H}]$, where $\mathrm{X}$ represents one given element. Four groups were identified and then analysed in terms of their relations with $[\mathrm{Fe} / \mathrm{H}]$, age, $[\mathrm{Ba} / \mathrm{Fe}]$, kinematics, and Galactic orbits.

Despite the small range in metallicity, our sample stars cover a broad range in age, and possible trends with age were traced. The relation between $[\mathrm{X} / \mathrm{Fe}]$ and $[\mathrm{Ba} / \mathrm{Fe}]$ for a few elements has also been considered given the Ba-rich nature of some of our stars. In particular, we investigated previous correlations with $\mathrm{Na}$ and $\mathrm{Cu}$ suggested by Castro et al. (1999). Finally, considering the results of Rocha-Pinto et al. (2006) that, on average, metalpoor and old stars tend to have larger $\left|R_{\mathrm{m}}-R_{\odot}\right|$ (where $R_{\mathrm{m}}$ is the mean orbital distance from the Galactic centre), we have looked for any relations involving the stellar groups of the clustering analysis and the kinematic and orbital parameters of the sample.

Though limited in size, our sample was carefully built up to undergo an homogeneous and detailed analysis, based on spectra with high resolution and high signal-to-noise ratio $(\mathrm{S} / \mathrm{N})$, in order to achieve a precision as high as possible in our determinations. Particular care was exercised to derive the stellar atmospheric parameters from different and independent criteria, in an attempt to limit the abundance uncertainties as much as possible.

In Sect. 2 we describe the observations and the reduction process. In Sect. 3 we present the methods used to derive the atmospheric parameters and the chemical abundances. The stellar evolution, kinematics, and orbits are presented in Sect. 4. The tree clustering method is described in Sect. 5, and all the results are discussed in Sect. 6. Finally, we present our conclusions in Sect. 7.

\section{Observations and data reduction}

The sample stars were selected from the Bright Star (Hoffleit \& Jaschek 1982) and Hipparcos (ESA 1997) catalogues according to the following conditions:

i) solar neighbourhood stars in a distance $\leq 40 \mathrm{pc}$;

ii) stars brighter than $V=6.5$ and with declination $<+20^{\circ}$;

iii) stars with effective temperature and metallicity distributed over about $5500 \leq T_{\text {eff }} \leq 6100 \mathrm{~K}$ and $-0.3 \leq[\mathrm{Fe} / \mathrm{H}] \leq$ 0.3 dex, respectively, which represents the colour index range $0.52 \leq(B-V) \leq 0.78$ from the $(B-V)$ calibration described in Sect. 3.4 (Eq. (3)); and

iv) stars with no information of duplicity (capable of significantly affecting the spectroscopy) available in the astrometric and spectroscopic binary catalogues of Hoffleit \& Jaschek (1982), Warren \& Hoffleit (1987), Batten et al. (1989), and Duquennoy \& Mayor (1991); the possibility of duplicity was afterward revised in the Washington Double Star Catalogue (Mason et al. 2001) and in the survey of Raghavan et al. (2010), and no close-in companions that could affect our analysis were found.

Based on these criteria, $99 \mathrm{~F}, \mathrm{G}$, and $\mathrm{K}$ dwarfs and subgiants were selected, out of which 25 stars were observed and analysed in this work. Our sample, shown in Table 1, contains F and $\mathrm{G}$ dwarf and subgiants stars from the thin disc stellar population, excepting the star HD 50806, which is probably in the transition between thin and thick discs.

The observations were carried out at the Cerro Tololo Inter-American Observatory (CTIO, Chile) in two different runs: i) 15 stars were observed in March 25-26, 1994 using the Cassegrain échelle spectrograph mounted on the $4 \mathrm{~m}$ telescope, with the red camera, $140 \mu \mathrm{m}$ slit, and Tek CCD detector of $1024 \times 1024$ pixels $(24 \times 24 \mu \mathrm{m}$ pixel size $)$, and with a gain of $1 \mathrm{e}^{-} /$ADU; the spectra have resolution $R \sim 29000$ and cover the wavelength range 4370-6870 A divided into 46 orders; and ii) 10 stars were observed in November 8-15, 1997 using the bench-mounted échelle spectrograph and a $750 \mathrm{~mm}$ folded Schmidt camera attached to the $1.5 \mathrm{~m}$ telescope; the same CCD was used; the spectra have resolution $R \sim 46000$ and cover the wavelength range 4550-6520 A divided into 37 orders.

The two subsamples, although observed in different conditions, were both selected based on the same criteria and will be treated as an homogeneous single sample. The spectra collected in the first run have, on average, signal-to-noise ratio $(S / N=395 \pm 60)$ slightly larger than those of the second one $(S / N=320 \pm 90)$ and, despite having smaller resolution, may provide smaller uncertainties in some parameters estimated here. Nevertheless, all spectra have $S / N>200$ in the blue region, which warranties spectral line profiles good enough to the equivalent width measurements. Any differences in the error estimates are discussed throughout the paper whenever needed.

Two spectra of the sunlight reflected by Ganymede were also observed, one in each run. The $\mathrm{S} / \mathrm{N}$ were estimated using continuum windows in the spectra selected by inspection of the solar flux atlas of Kurucz et al. (1984) (hereafter the Solar Flux Atlas) and the solar line identifications catalogue of Moore et al. (1966) (hereafter the Solar Lines Catalogue). The mean values $\langle\mathrm{S} / \mathrm{N}\rangle$ measured in the wavelength range 4500-5000 $\AA$ are listed in Table 1. For larger wavelengths the $\mathrm{S} / \mathrm{N}$ are even higher, approaching twice that for $\lambda 4500$ in the range 6000-6500 $\AA$. 
Table 1. The 25 sample stars.

\begin{tabular}{lccc}
\hline \hline Object & $V$ & Sp. type & $\langle S / N\rangle$ \\
\hline \multicolumn{4}{c}{ First run (March 26-25, 1994): } \\
Ganymede & 5.10 & G2 V & 450 \\
HD 20807 & 5.24 & G0 V & 430 \\
HD 43834 & 5.08 & G7 V & 370 \\
HD 84117 & 4.93 & F8 V & 410 \\
HD 102365 & 4.89 & G2 V & 370 \\
HD 112164 & 5.89 & F9 V & 400 \\
HD 114613 & 4.85 & G3 V & 400 \\
HD 115383 & 5.19 & G0 V & 320 \\
HD 115617 & 4.74 & G7 V & 370 \\
HD 117176 & 4.97 & G5 V & 410 \\
HD 128620 & -0.01 & G2 V & 450 \\
HD 141004 & 4.42 & G0 V & 520 \\
HD 146233 & 5.49 & G2 V & 420 \\
HD 147513 & 5.37 & G5 V & 290 \\
HD 160691 & 5.12 & G3 IV-V & 400 \\
HD 188376 & 4.70 & G5 IV & 310 \\
\hline \multicolumn{5}{c}{ Second run (November 8-15, 1997): } \\
Ganymede & 5.10 & G2 V & 410 \\
HD 1835 & 6.39 & G3 V & 230 \\
HD 26491 & 6.37 & G1 V & 310 \\
HD 33021 & 6.15 & G1 IV & 240 \\
HD 39587 & 4.39 & G0 V & 510 \\
HD 50806 & 6.05 & G5 V & 300 \\
HD 53705 & 5.56 & G0 V & 370 \\
HD 177565 & 6.15 & G6 V & 290 \\
HD 181321 & 6.48 & G2 V & 220 \\
HD 189567 & 6.07 & G2 V & 260 \\
HD 196761 & 6.36 & G8 V & 400 \\
\hline \multicolumn{4}{c}{} \\
\hline
\end{tabular}

Notes. The mean values of $\mathrm{S} / \mathrm{N}$ measured in continuum windows around $\lambda \lambda$ 4500-5000 $\AA$ are listed for the stars and for the two sunlight spectra (Ganymede).

The spectra were reduced using $\operatorname{IRAF}^{1}$ routines for order identification and extraction, background subtraction (including bias and scattered light), flat-field correction, wavelength calibration, radial-velocity shift correction, and flux normalisation. The wavelength calibration was performed onto the stellar spectra themselves using lines selected by inspection of the Solar Flux Atlas and the Solar Lines Catalogue. The normalisation of the continuum is a very delicate and relevant step in the analysis procedure, since the accuracy of the equivalent width measurements is very sensitive to a faulty determination of the continuum level. Therefore, continuum windows free from telluric or photospheric lines were carefully selected also based on the Solar Flux Atlas and the Solar Lines Catalogue.

\section{Spectroscopic analysis}

A differential spectroscopic analysis relative to the Sun was performed to determine the atmospheric parameters and the chemical abundance of several elements in our sample. The analysis was based on the equivalent widths of atomic lines measured in the spectra, and on the spectral synthesis of carbon atomic and molecular lines. The two groups of stars $(15$ observed in the first and 10 in the second run) were treated in comparison with the Ganymede spectrum of their respective run.

\footnotetext{
${ }^{1}$ Image Reduction and Analysis Facility, distributed by the National Optical Astronomy Observatories (NOAO), USA.
}

\subsection{Equivalent widths and atomic line parameters}

Atomic lines of the elements $\mathrm{Na}, \mathrm{Mg}, \mathrm{Si}, \mathrm{Ca}, \mathrm{Sc}, \mathrm{Ti}, \mathrm{V}, \mathrm{Cr}, \mathrm{Mn}$, $\mathrm{Fe}, \mathrm{Co}, \mathrm{Ni}, \mathrm{Cu}, \mathrm{Zn}, \mathrm{Sr}, \mathrm{Y}, \mathrm{Zr}, \mathrm{Ba}, \mathrm{Ce}, \mathrm{Nd}$, and $\mathrm{Sm}$ were selected throughout the spectral range for equivalent width (EW) measurements. The lines were chosen based on the Solar Flux Atlas and the Solar Lines Catalogue, selecting only those for which the profiles were sufficiently clean from blends in order to provide reliable measurements. For both solar and stellar spectra, the EW values of more than 7500 lines of these elements (about 300 lines per star) were measured by hand by Gaussian function fit using IRAF routines.

Strong line profiles are better described by Voigt functions than by Gaussian functions. We show in Fig. 1 (left panel) a comparison of the EWs measured in this work in the Ganymede spectrum of the second observation run (Gany 2) by Gaussian function fit to those measured in the Solar Flux Atlas $(R>500000$ and $S / N \sim 3000$ ) by Voigt function fit (Meylan et al. 1993). A similar diagram was obtained using the Ganymede spectrum of the first run (Gany 1) and the following relations represent the linear least square regressions fitted to both diagrams:

$$
\begin{aligned}
& E W_{\text {Solar Flux Atlas }}=(0.3 \pm 0.6)+(1.065 \pm 0.012) E W_{\text {Gany } 1} \\
& E W_{\text {Solar Flux Atlas }}=(0.8 \pm 0.5)+(1.075 \pm 0.010) E W_{\text {Gany } 2}
\end{aligned}
$$

where $\mathrm{EW}$ is given in $\mathrm{m \AA}$. The standard deviations and the cross-correlation coefficients are, respectively, $\sigma=3.4 \mathrm{~m} \AA$ and $r=0.991$ for Eq. (1), and $\sigma=2.7 \mathrm{m \AA}$ and $r=0.994$ for Eq. (2). Therefore, to reduce possible systematic uncertainties and provide direct comparison with other works, all our EWs were transformed to a common system using the regression coefficients of these equations (the constant terms have no statistical significance within $2 \sigma$ ). The regressions were derived in order to have a direct transformation to the Solar Flux Atlas system. We also did the converse (using $E W_{\text {Ganymede }}$ vs. $E W_{\text {Solar Flux Atlas }}$ diagrams) for comparison, and the resulting regressions are comparable with those of Eqs. (1) and (2), within $1 \sigma$.

The wavelength and lower-level excitation potential $(\chi)$ of the atomic lines used were taken from the Solar Lines Catalogue. The oscillator strengths $(g f)$ were computed using a solar model atmosphere applied to the EWs of Ganymede (converted using Eqs. (1) and (2)) in order to provide the standard solar abundances of Grevesse \& Noels (1993). The adopted solar abundances are of course inconsequential in a differential analysis.

The solar and stellar model atmospheres were computed with a code kindly supplied by Dr. Monique Spite (Meudon Observatory, Paris) that interpolates the model-atmosphere grid from Edvardsson et al. (1993). We used an updated version of the original code from Spite (1967). The fundamental atmospheric parameters (effective temperature $T_{\text {eff }}$, metallicity $[\mathrm{Fe} / \mathrm{H}]$, surface gravity $\log g$, and micro-turbulence velocity $\xi$ ) and the population ratio of helium and hydrogen atoms $\left(n_{\mathrm{He}} / n_{\mathrm{H}}\right)$ are taken as input. For the Sun, the adopted parameters are $T_{\mathrm{eff}}=5777 \mathrm{~K}$, $\log g=4.44, \xi=1.3 \mathrm{~km} \mathrm{~s}^{-1}, n_{\mathrm{He}} / n_{\mathrm{H}}=0.1$, and $\log \epsilon_{\odot}=7.50$ (the solar Fe abundance).

The spectral lines used and their parameters are listed in Table 8, in which the EWs are the raw values (before the conversion). We do not list the EWs of the other stars but they are available upon request.

The atomic lines of the elements $\mathrm{Mg}, \mathrm{Sc}, \mathrm{V}, \mathrm{Mn}, \mathrm{Co}$, and $\mathrm{Cu}$ have important hyperfine structure (HFS). Their $g f$ values, 

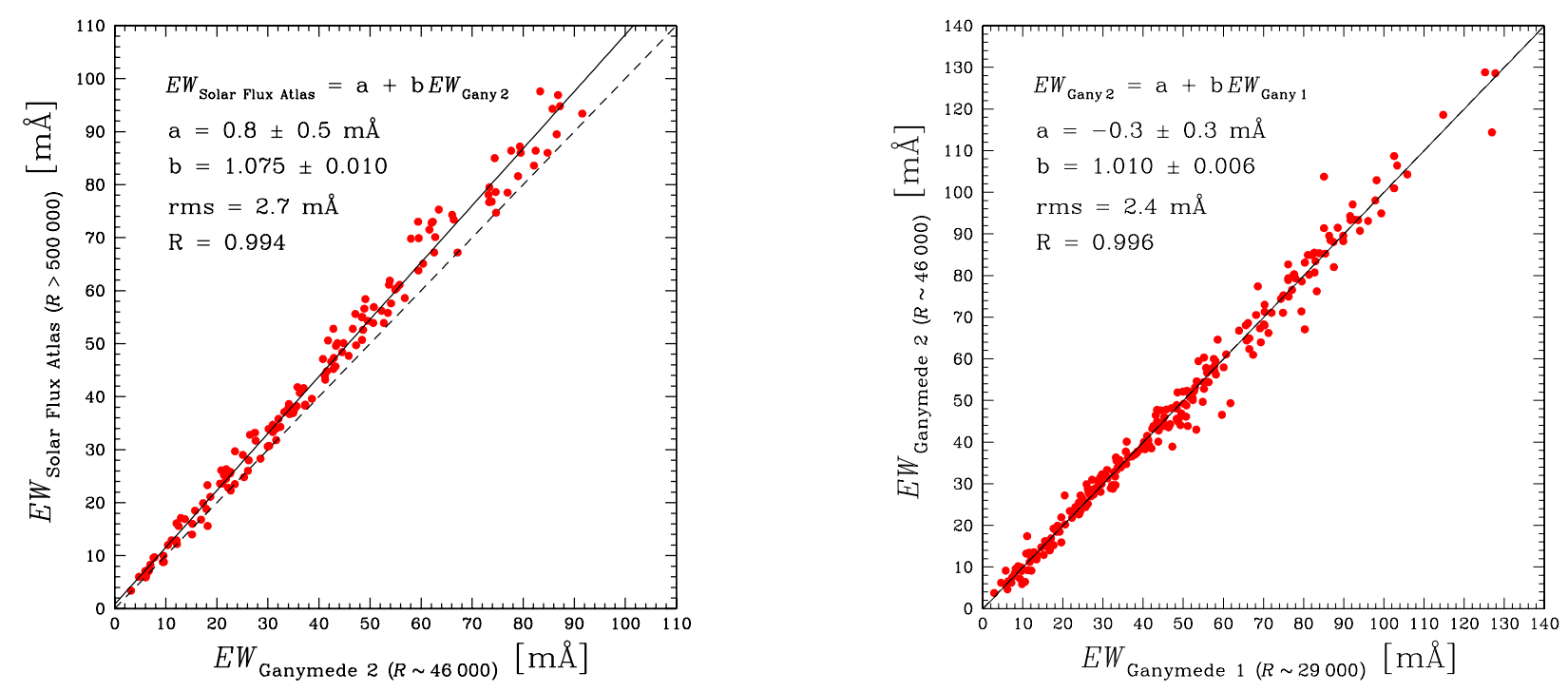

Fig. 1. Left panel: comparison of the EWs measured in this work in the Ganymede spectrum (second observation run) by Gaussian function fit to those measured by Meylan et al. (1993) in the Solar Flux Atlas by Voigt function fit; filled circles represent 145 lines of several elements; the linear regression (solid line) is the same expressed by Eq. (2). Right panel: comparison of the EWs listed in Table 8 of all lines in the Ganymede spectra in common to both runs after the conversions according to Eqs. (1) and (2); the coefficients of the linear regression (solid line) are shown.

listed in Table 9, were taken from Steffen (1985) and also revised according to the EWs of Ganymede and the standard solar abundances of Grevesse \& Noels (1993), as done for the other elements. For the elements $\mathrm{Zn}, \mathrm{Sr}, \mathrm{Y}, \mathrm{Zr}, \mathrm{Ce}, \mathrm{Ba}$, and $\mathrm{Nd}$, for which theoretical HFS exist, either their effects are negligible or the spectral lines used are too weak to depend on the HFS assumption. For the elements without HFS data listed in Steffen (1985), values of neighbouring multiplets were adopted. The only exception was the $\mathrm{Mg}$ line at $\lambda 5785.285$, for which no HSF information was available. Its $g f$ value, listed in Table 8, was obtained in the same way as those lines without HFS. This is not a strong line $(E W<60 \mathrm{~m} \AA)$ so that the error induced in the $\mathrm{Mg}$ abundance determination is not important. del Peloso et al. (2005) have recently shown that for Mn and Co the differences in their abundances computed using different values of HFS are not greater than 0.10 dex.

The HFS of $\mathrm{Ba}$, and also its isotopic splitting, are of some importance only for the line at $\lambda 6496.9$, and can be neglected for the lines at $\lambda 5853.7$ and $\lambda 6141.7$ (see Korotin et al. 2011). However, for the 25 stars of our sample we have found a good agreement among the abundances yielded by the three lines, with a mean standard deviation of 0.07 dex. Moreover, a test performed using only $\lambda 5853.7$ and $\lambda 6141.7$ indicated that the global behaviour and trends found in the abundance diagrams, as well as all our conclusions involving the Ba results would not change if only these two lines were used.

Concerning the fact that we used two spectrographs with different spectral resolutions, we performed a test in which we degraded the spectrum of Ganymede and of the metal-rich star HD 1835, both observed in the second observation run, to match the resolution of the first run. New values of EWs were then obtained, and no systematic differences were found when they are compared with the original measurements. Moreover, a comparison of the EWs of Ganymede listed in Table 8 and converted according to Eqs. (1) and (2) (see Fig. 1, right panel) demonstrates that the equivalent widths of the two observation runs were properly transformed to a common system, reinforcing our assumption of an homogeneous analysis.

\subsection{Derivation of atmospheric parameters}

In order to determine the fundamental atmospheric parameters we developed a code that iteratively calculates these parameters for a given star based on initial input values. The so called excitation effective temperature $\left(T_{\text {eff }}^{\text {exc }}\right)$ was calculated through the excitation equilibrium of neutral iron by removing any dependence in $\mathrm{a}[\mathrm{Fe} \mathrm{I} / \mathrm{H}]$ vs. $\chi$ diagram. The micro-turbulence velocity was obtained by removing the dependence of $\left[\mathrm{Fe}_{\mathrm{I}} / \mathrm{H}\right]$ on $\mathrm{EW}$, and the ionisation surface gravity $\left(\log g_{\text {ion }}\right)$ was computed through the ionisation equilibrium between $\mathrm{Fe}_{\mathrm{I}}$ and Fe II. Finally, the metallicity was yielded by the EW of Fe I lines.

The temperature used in our abundance analyses was the excitation effective temperature, which is a better representation of the temperature stratification of the line forming layers. In order to compute the stellar luminosity with more reliability by also accounting for any consequence due to small LTE departures, we also considered two other temperature indicators, which are described in Sect. 3.4.

Table 2 lists the spectroscopic atmospheric parameters of the programme stars. An estimate of their uncertainties was performed based on the analysis of HD 146233 and HD 26491, which are representative stars in our sample (from the first and second runs, respectively) with regard to their parameters and quality of the spectroscopic data. For both stars we have similar errors and they were obtained as follows:

i) The uncertainty in $T_{\mathrm{eff}}^{\mathrm{exc}}$ is related to the standard error of the angular coefficient of the linear regression fitted to the $[\mathrm{Fe} \mathrm{I} / \mathrm{H}]$ vs. $\chi$ diagram. The temperature is changed until this coefficient is of the same order of its error. The difference between the best value and the previous one from the last iteration provides the uncertainty $\sigma\left(T_{\text {eff }}^{\text {exc }}\right)=30 \mathrm{~K}$;

ii) The uncertainty in metallicity is the standard deviation of the abundance yielded by individual $\mathrm{Fe}_{\mathrm{I}}$ lines, which is $\sigma([\mathrm{Fe} / \mathrm{H}])=0.05 \mathrm{dex}$

iii) To estimate the uncertainty in $\log g_{\text {ion }}$, its value is changed until the difference between the averaged abundance yielded by $\mathrm{Fe}_{\mathrm{I}}$ and $\mathrm{Fe}$ II lines is of the order of their 
Table 2. Atmospheric parameters from our spectroscopic analysis.

\begin{tabular}{lccccc}
\hline \hline Star & $\begin{array}{c}T_{\text {eff }}^{\text {exc }} \\
{[\mathrm{K}]}\end{array}$ & $\log g_{\text {ion }}$ & $\log g_{\text {evol }}$ & $\begin{array}{c}\xi \\
{\left[\mathrm{km} \mathrm{s}^{-1}\right]}\end{array}$ & {$[\mathrm{Fe} / \mathrm{H}]$} \\
\hline Sun & 5777 & 4.44 & 4.44 & 1.30 & 0.00 \\
HD 1835 & 5890 & 4.52 & 4.49 & 1.66 & 0.21 \\
HD 20807 & 5878 & 4.51 & 4.44 & 1.33 & -0.22 \\
HD 26491 & 5820 & 4.38 & 4.28 & 1.38 & -0.09 \\
HD 33021 & 5750 & 4.14 & 4.05 & 1.40 & -0.20 \\
HD 39587 & 6000 & 4.52 & 4.48 & 1.72 & 0.00 \\
HD 43834 & 5630 & 4.47 & 4.44 & 1.23 & 0.11 \\
HD 50806 & 5610 & 4.12 & 4.03 & 1.36 & 0.02 \\
HD 53705 & 5810 & 4.32 & 4.26 & 1.34 & -0.22 \\
HD 84117 & 6074 & 4.20 & 4.27 & 1.56 & -0.06 \\
HD 102365 & 5643 & 4.47 & 4.40 & 1.04 & -0.28 \\
HD 112164 & 6031 & 4.05 & 3.87 & 1.79 & 0.32 \\
HD 114613 & 5706 & 3.97 & 3.87 & 1.55 & 0.15 \\
HD 115383 & 6126 & 4.43 & 4.26 & 1.61 & 0.23 \\
HD 115617 & 5587 & 4.41 & 4.42 & 1.22 & 0.00 \\
HD 117176 & 5587 & 4.13 & 3.91 & 1.36 & -0.04 \\
HD 128620 & 5857 & 4.44 & 4.31 & 1.45 & 0.23 \\
HD 141004 & 5926 & 4.28 & 4.18 & 1.49 & 0.03 \\
HD 146233 & 5817 & 4.45 & 4.42 & 1.32 & 0.05 \\
HD 147513 & 5891 & 4.63 & 4.48 & 1.41 & 0.04 \\
HD 160691 & 5777 & 4.32 & 4.19 & 1.40 & 0.27 \\
HD 177565 & 5630 & 4.42 & 4.43 & 1.24 & 0.08 \\
HD 181321 & 5810 & 4.34 & 4.56 & 2.30 & -0.06 \\
HD 188376 & 5514 & 3.71 & 3.61 & 1.55 & 0.00 \\
HD 189567 & 5700 & 4.44 & 4.32 & 1.22 & -0.27 \\
HD 196761 & 5410 & 4.44 & 4.49 & 1.08 & -0.32 \\
\hline
\end{tabular}

Notes. For comparison, the evolutionary surface gravity computed as described in Sect. 4.1 (Eq. (7)) is also shown.

internal errors $(\sim 0.05 \mathrm{dex})$, which led to an uncertainty $\sigma\left(\log g_{\text {ion }}\right)=0.13$ dex;

iv) The uncertainty in the micro-turbulence velocity is estimated regarding the $[\mathrm{Fe} \mathrm{I} / \mathrm{H}]$ vs. EW diagram. The $\xi$ value is modified until the angular coefficient of the regression is of the same order of its error. An uncertainty $\sigma(\xi)=0.04 \mathrm{~km} \mathrm{~s}^{-1}$ was found for this parameter.

The spectroscopic atmospheric parameters were used to compute the model atmospheres, which in turn are required in the abundance determination. In our analysis, we used the modelatmosphere grid derived by Edvardsson et al. (1993) for stars with effective temperatures from 5250 to $6000 \mathrm{~K}$, surface gravity from 2.5 to 5.0 dex, and metallicity from -2.3 to +0.3 dex (with small extrapolations when needed). These are 1D, plane-parallel, constant flux, line-blanketed, and LTE models computed over 45 layers.

The model atmospheres are, essentially, subject to errors in the atmospheric parameters, in the LTE simplifications, and in the thermal homogeneity assumption. However, the effects of non-LTE and thermal inhomogeneities are hopefully minor for the elements and the stellar types studied here, being more important for low metallicity and low surface gravity stars (Edvardsson et al. 1993; Asplund 2005). Possible errors induced by such simplified assumptions are dominated by other sources of uncertainties. In addition, in a differential analysis, the errors in the stellar atmospheric structure are of second order.

We also investigated what would be the effects on the derived abundances if another model-atmosphere grid were used. We compared Edvardsson and Kurucz models and, using the same equivalent widths, $g f$ values, and atmospheric parameters obtained from a solar spectrum, we found that the differences in abundance $[\mathrm{X} / \mathrm{Fe}]$ for most of the elements are of the order of 0.03 dex or smaller, achieving a maximum of 0.07 dex. We note that these values are for the Sun and represent the differences between the two sets of model atmospheres. The effects of these differences when computing the stellar abundances relative to the Sun are minimised in a differential approach.

\subsection{Abundance determination and their uncertainties}

The abundance of the elements studied was determined using an adapted version of a code also supplied by Dr. Monique Spite. The code takes into account the solar $g f$ values and the stellar model atmospheres (computed using the atmospheric parameters of each star) to calculate the abundances that fits the equivalent widths measured in the spectra (transformed according to the procedure described in Sect. 3.1). The results of this abundance determination are presented and discussed in Sect. 6 .

The main sources of uncertainties in the abundance determination come from the errors in the EWs (the most impor$\operatorname{tant}$ ), the $g f$ values, the atmospheric parameters, and the adopted model atmospheres (these two latter are discussed in Sect. 3.2).

The uncertainties in the EWs were estimated as follows: by plotting the diagram $E W_{\mathrm{HD} 26491}$ vs. $E W_{\text {Ganymede }}$ and computing the standard deviation of the linear regression, we obtained $\sigma=2.9 \mathrm{~m} \AA$. The solar EWs were measured in the surrogate spectrum of the Sun collected under the same circumstances as for the programme stars. Therefore, we assumed that $\sigma$ is a quadratic sum of the errors in EW of both objects and that they are similar to each other. Thus, for the star HD 26491 the value of $\sigma(E W)$ is $\sigma / \sqrt{2}=2.1 \mathrm{~m} \AA$. Similarly, for the star HD 146233 we obtained $\sigma(E W)=1.7 \mathrm{~m} \AA$. These values were adopted to represent the uncertainties in EW of the two observation runs. Because the solar $g f$ values were computed to reproduce solar the equivalent widths, the errors in EW contribute twice to the total uncertainty, with approximately the same magnitude.

Each one in turn, EW, $T_{\text {eff }}^{\text {exc }},[\mathrm{Fe} / \mathrm{H}], \xi$, and $\log g_{\text {ion }}$ are changed by $1 \sigma$ in the sense of increasing the abundance ratios and new abundances are computed for each element. The differences between new and previous abundance values provide the errors induced by each parameter and a quadratic sum of these errors yields the total uncertainty in the elemental abundance ratios. The estimated errors $\left(\sigma_{\text {est }}\right)$ are listed in Table 3 for both HD 146233 and HD 26491, and they are compared to the dispersions around the mean $\left(\sigma_{\text {disp }}\right)$ for elements with at least five lines measured in the spectra. For these elements, the larger values were adopted to represent the errors $\sigma([\mathrm{X} / \mathrm{Fe}])$ in each observation run. Otherwise, $\sigma_{\text {est }}$ was adopted.

\subsection{Photometric and $\mathrm{H} \alpha$ effective temperatures}

The effective temperature of the sample stars were also obtained using some photometric calibrations, providing the photometric effective temperature $\left(T_{\mathrm{eff}}^{\mathrm{phot}}\right)$. These calibrations, derived by Porto de Mello et al. (in prep.) for the $(B-V),\left(B_{\mathrm{T}}-V_{\mathrm{T}}\right),(b-y)$, and $\beta$ colour indices, are given by the following equations:

$$
\begin{aligned}
& T_{\mathrm{eff}(B-V)}^{\mathrm{phot}}=7747-3016(B-V)\{1-0.15[\mathrm{Fe} / \mathrm{H}]\} \\
& T_{\mathrm{eff}\left(B_{\mathrm{T}}-V_{\mathrm{T}}\right)}^{\mathrm{phot}}=7551-2406\left(B_{\mathrm{T}}-V_{\mathrm{T}}\right)\{1-0.2[\mathrm{Fe} / \mathrm{H}]\} \\
& T_{\mathrm{eff}(b-y)}^{\mathrm{phot}}=8481-6516(b-y)\{1-0.09[\mathrm{Fe} / \mathrm{H}]\} \\
& T_{\mathrm{eff}(\beta)}^{\mathrm{phot}}=11654\{\beta-2.349\}^{0.5}
\end{aligned}
$$


Table 3. A comparison between the estimated abundance errors $\sigma_{\text {est }}$ (see Sects. 3.3 and 3.5) and the dispersions of these abundances around the mean $\sigma_{\text {disp }}$ for elements with $N=5$ or more lines measured.

\begin{tabular}{lcccccc}
\hline \hline \multirow{2}{*}[\mathrm{X}/\mathrm{Fe}]{} & \multicolumn{3}{c}{ HD 146233 } & \multicolumn{3}{c}{ HD 26491 } \\
& $\sigma_{\text {est }}$ & $\sigma_{\text {disp }}$ & $N$ & $\sigma_{\text {est }}$ & $\sigma_{\text {disp }}$ & $N$ \\
\hline $\mathrm{C}$ & 0.07 & - & 2 & 0.07 & - & 2 \\
$\mathrm{Na}$ & 0.03 & - & 2 & 0.06 & - & 2 \\
$\mathrm{Mg}$ & 0.03 & - & 4 & 0.06 & - & 4 \\
$\mathrm{Si}$ & 0.05 & 0.06 & 11 & 0.06 & 0.03 & 17 \\
$\mathrm{Ca}$ & 0.03 & 0.04 & 6 & 0.05 & 0.05 & 13 \\
$\mathrm{Sc}$ & 0.06 & 0.03 & 6 & 0.09 & 0.03 & 13 \\
$\mathrm{Ti}$ & 0.07 & 0.04 & 24 & 0.10 & 0.04 & 38 \\
$\mathrm{~V}$ & 0.07 & 0.07 & 8 & 0.11 & 0.04 & 11 \\
$\mathrm{Cr}$ & 0.06 & 0.04 & 14 & 0.07 & 0.04 & 29 \\
$\mathrm{Mn}$ & 0.04 & 0.05 & 8 & 0.06 & 0.04 & 11 \\
$\mathrm{Co}$ & 0.07 & 0.05 & 9 & 0.11 & 0.04 & 12 \\
$\mathrm{Ni}$ & 0.04 & 0.03 & 23 & 0.07 & 0.04 & 26 \\
$\mathrm{Cu}$ & 0.04 & - & 3 & 0.07 & - & 3 \\
$\mathrm{Zn}$ & 0.05 & - & 1 & 0.06 & - & 1 \\
$\mathrm{Sr}$ & 0.06 & - & 1 & 0.07 & - & 1 \\
$\mathrm{Y}$ & 0.07 & 0.05 & 5 & 0.09 & 0.05 & 5 \\
$\mathrm{Zr}$ & 0.09 & - & 3 & 0.08 & - & 3 \\
$\mathrm{Ba}$ & 0.06 & - & 3 & 0.08 & - & 3 \\
$\mathrm{Ce}$ & 0.07 & 0.07 & 5 & 0.12 & - & 3 \\
$\mathrm{Nd}$ & 0.16 & - & 2 & 0.12 & - & 2 \\
$\mathrm{Sm}$ & 0.09 & - & 1 & 0.15 & - & 1 \\
\hline
\end{tabular}

Notes. For each element of each observation run the larger value was adopted to represent the errors $\sigma([\mathrm{X} / \mathrm{Fe}])$.

for $T_{\text {eff }}$ given in $\mathrm{K}$. The standard deviations of these calibrations are $\sigma=65,64,55$, and $70 \mathrm{~K}$, respectively.

The $(B-V)$ and $\left(B_{\mathrm{T}}-V_{\mathrm{T}}\right)$ colour indices of our stars were taken from the Hipparcos Catalogue, and $(b-y)$ and $\beta$ from the literature (see Table 4), when available. For the star HD 33021, the $\beta$ values adopted are only from Perry et al. (1987) because these authors made 41 measurements of this index. For the star HD 50806, only one reference for the $\beta$ index was found, and the effective temperature from this index strongly disagrees with that obtained from the other colours and we thus discarded it.

Table 4 lists the colour indices used and the photometric effective temperature ( $\left.T_{\mathrm{eff}}^{\text {phot }}\right)$ obtained, which is a mean of the temperatures computed using the four calibrations, weighted by their variances. The references for $(b-y)$ and $\beta$ are also listed. The values of $(b-y)$ from Gronbech \& Olsen (1976), Olsen (1983), Twarog (1980), and Schuster \& Nissen (1988) were converted to the Olsen (1993) system according to equations provided by the latter author.

The star HD 128620 is the primary component $(V=-0.01)$ of the $\alpha$ Cen triple system. The $T_{\text {eff }}$ determination for very bright stars using photometric colours is normally considered risky due to systematic effects that may affect the results (such as nonlinearity and detector dead time) and also, in the case of this system, due to a possible contamination by the companion. For this reason, we preferred do not include this star in our $T_{\text {eff }}$ estimates based on the photometric indices. Nevertheless, our spectroscopic determination for $\alpha \mathrm{Cen} \mathrm{A}, T_{\mathrm{eff}}^{\mathrm{exc}}=5857 \pm 30 \mathrm{~K}$, is in good agreement with the photometric determination performed by Porto de Mello et al. (2008), $T_{\text {eff }}=5794 \pm 34 \mathrm{~K}$.

Concerning the uncertainties in $T_{\mathrm{eff}}^{\mathrm{phot}}$, on the one hand, the internal error of the weighted mean, computed using the standard deviations in the four photometric calibrations, is $31 \mathrm{~K}$. On the other hand, the mean value of the standard deviations around $T_{\mathrm{eff}}^{\text {phot }}$ (i.e., the dispersion of the four values of temperature around the weighted mean) is $41 \mathrm{~K}$. Therefore, in this work we adopted $\sigma\left(T_{\text {eff }}^{\text {phot }}\right)=40 \mathrm{~K}$ as the internal uncertainty in our photometric effective temperatures. This uncertainty intrinsically takes into account the errors in the colour indices themselves.

The stellar effective temperature can also be estimated by modelling the wing profile of the $\mathrm{H} \alpha$ line, which is very sensitive to changes in this parameter. Lyra \& Porto de Mello (2005) applied this method to solar neighbourhood stars, and values of $T_{\mathrm{eff}}^{\mathrm{H} \alpha}$ for our sample were used as a third $T_{\mathrm{eff}}$ indicator, with an uncertainty $\sigma\left(T_{\text {eff }}^{\mathrm{H} \alpha}\right)=50 \mathrm{~K}$.

\subsection{Carbon abundance from spectral synthesis}

The carbon abundance was derived using the spectral synthesis method applied to molecular lines of electronic-vibrational band heads of the $C_{2}$ Swan System at $\lambda 5128$ and $\lambda 5165$, and also to $\mathrm{C}$ atomic lines at $\lambda 5052.2$ and $\lambda 5380.3$. To reproduce the atomic and molecular absorption lines in the observed spectra of the sample stars, the MOOG spectral synthesis code ${ }^{2}$, developed by Chris Sneden (University of Texas, USA), was used. The synthetic spectra were computed in steps of $0.02 \AA$, also taking into account the continuum opacity contribution in ranges of $0.5 \AA$. The Unsöld approximation multiplied by 6.3 was adopted in the calculations of the line damping parameters.

The model atmospheres are the same used in the spectroscopic analysis. They also include the micro-turbulence velocity and the elemental abundances of each star, both assumed to be constant in all layers. For any element $X$ for which no abundance was determined in this work, we adopted the metallicity of the respective star to set the $[\mathrm{X} / \mathrm{H}]$ ratio.

The atomic and molecular line parameters used to compute the synthetic spectra are: the central wavelength, the $g f$ values, the lower-level excitation potential, and the constant of dissociation energy $D_{0}$ (only for molecular features). Atomic and molecular data were taken, respectively, from the Vienna Atomic Line Database - VALD (Kupka et al. 1999, 2000; Piskunov et al. 1995; Ryabchikova et al. 1997) and from Kurucz (1992). In addition to $\mathrm{C}_{2}$, the spectral regions studied also include $\mathrm{MgH}$ molecular features that may contribute to the continuum formation. The oscillator strengths of $\mathrm{C}_{2}$ and $\mathrm{MgH}$ lines were revised according to the normalisation of the Hönl-London factors (Whiting \& Nicholls 1974).

To account for the spectral line broadening, the synthetic spectra were computed by means of the convolution with three input parameters: i) the spectroscopic instrumental broadening; ii) the limb darkening of the stellar disc; and iii) a composite of velocity fields, such as rotation velocity and macro-turbulence broadening, named $V_{\text {broad }}$. The instrumental broadening was estimated by means of the FWHM of thorium lines present in Th-Ar spectra observed at the CTIO. The linear limb-darkening coefficient (on average $u \sim 0.7$ for all the sample stars) was individually estimated by interpolation of $T_{\mathrm{eff}}^{\mathrm{exc}}$ and $\log g_{\mathrm{ion}}$ in Table 1 of Díaz-Cordovéz et al. (1995). As a first estimate of $V_{\text {broad }}$, the projected rotation velocity $(v \sin i)$ of the stars was used, which was computed based on the profile of four isolated $\mathrm{Fe}_{\mathrm{I}}$ lines $(\lambda 5852.2, \lambda 5855.1, \lambda 5856.1$, and $\lambda 5859.6)$ in the spectra. Small corrections in $V_{\text {broad }}$ were applied when needed according to an eye-trained inspection of the synthetic spectra. The final values are listed in Table 5, where they can be compared to the stellar age and the chromospheric activity level.

${ }^{2}$ http://www.as.utexas.edu/ chris/moog.html 
Table 4. Colour indices and photometric effective temperatures (given in K).

\begin{tabular}{|c|c|c|c|c|c|c|c|c|c|c|}
\hline Star & $B-V$ & $B_{\mathrm{T}}-V_{\mathrm{T}}$ & $b-y^{1}$ & $\beta^{1}$ & $\begin{array}{c}T_{\mathrm{eff}}^{\mathrm{phot}} \\
(B-V)\end{array}$ & $\begin{array}{c}T_{\mathrm{eff}}^{\mathrm{phot}} \\
\left(B_{\mathrm{T}}-V_{\mathrm{T}}\right)\end{array}$ & $\begin{array}{c}T_{\mathrm{eff}}^{\text {phot }} \\
(b-y)\end{array}$ & $\begin{array}{c}T_{\text {eff }}^{\text {phot }} \\
(\beta)\end{array}$ & $\begin{array}{c}T_{\text {eff }}^{\text {phot }} \\
\text { (mean) }\end{array}$ & $T_{\mathrm{eff}}^{\mathrm{H} \alpha}$ \\
\hline HD 1835 & 0.659 & 0.758 & 0.420 & 2.606 & 5822 & 5804 & 5796 & 5908 & 5826 & 5846 \\
\hline HD 20807 & 0.600 & - & 0.380 & 2.592 & 5878 & - & 5956 & 5745 & 5876 & 5860 \\
\hline HD 26491 & 0.636 & 0.697 & 0.404 & 2.587 & 5803 & 5844 & 5827 & 5685 & 5797 & 5774 \\
\hline HD 33021 & 0.625 & 0.682 & 0.402 & 2.590 & 5805 & 5844 & 5814 & 5721 & 5800 & 5823 \\
\hline HD 39587 & 0.594 & 0.659 & 0.376 & 2.599 & 5955 & 5965 & 6031 & 5827 & 5956 & 5966 \\
\hline HD 43834 & 0.720 & 0.829 & 0.442 & 2.601 & 5611 & 5600 & 5629 & 5850 & 5662 & 5614 \\
\hline HD 50806 & 0.708 & 0.800 & 0.437 & - & 5618 & 5634 & 5639 & - & 5631 & 5636 \\
\hline HD 53705 & 0.624 & 0.685 & 0.396 & 2.595 & 5803 & 5830 & 5850 & 5780 & 5820 & 5821 \\
\hline HD 84117 & 0.530 & 0.581 & 0.339 & 2.622 & 6134 & 6136 & 6260 & 6089 & 6166 & 6188 \\
\hline HD 102365 & 0.660 & - & 0.408 & 2.588 & 5673 & - & 5755 & 5697 & 5714 & 5644 \\
\hline HD 112164 & 0.640 & 0.696 & 0.392 & 2.632 & 5909 & 5984 & 6000 & 6200 & 6015 & 5965 \\
\hline HD 114613 & 0.700 & 0.796 & 0.441 & - & 5683 & 5693 & 5646 & - & 5671 & 5732 \\
\hline HD 115383 & 0.590 & 0.644 & 0.371 & 2.615 & 6029 & 6073 & 6114 & 6011 & 6064 & 5952 \\
\hline HD 115617 & 0.710 & - & 0.434 & 2.582 & 5606 & - & 5653 & 5625 & 5631 & 5562 \\
\hline HD 117176 & 0.710 & 0.804 & 0.445 & 2.576 & 5593 & 5601 & 5571 & 5552 & 5580 & 5493 \\
\hline HD 128620 & - & - & - & - & - & - & - & - & - & 5820 \\
\hline HD 141004 & 0.600 & 0.672 & 0.382 & 2.606 & 5946 & 5944 & 5999 & 5908 & 5955 & 5869 \\
\hline HD 146233 & 0.650 & 0.736 & 0.398 & 2.596 & 5801 & 5798 & 5899 & 5792 & 5830 & 5790 \\
\hline HD 147513 & 0.620 & 0.703 & 0.391 & 2.609 & 5888 & 5873 & 5942 & 5942 & 5913 & 5840 \\
\hline HD 160691 & 0.700 & 0.786 & 0.432 & - & 5721 & 5762 & 5734 & - & 5739 & 5678 \\
\hline HD 177565 & 0.705 & 0.803 & 0.436 & 2.584 & 5646 & 5650 & 5660 & 5649 & 5652 & 5673 \\
\hline HD 181321 & 0.628 & 0.694 & 0.396 & - & 5836 & 5861 & 5887 & - & 5864 & 5845 \\
\hline HD 188376 & 0.750 & - & 0.458 & - & 5485 & - & 5497 & - & 5492 & 5436 \\
\hline HD 189567 & 0.648 & 0.718 & 0.410 & 2.583 & 5713 & 5730 & 5745 & 5637 & 5712 & 5697 \\
\hline HD 196761 & 0.719 & 0.828 & 0.441 & - & 5474 & 5431 & 5525 & - & 5482 & 5544 \\
\hline
\end{tabular}

Notes. The values of $(B-V)$ and $\left(B_{\mathrm{T}}-V_{\mathrm{T}}\right)$ were taken from the Hipparcos Catalogue. The H $\alpha$ effective temperatures from Lyra \& Porto de Mello (2005) are also listed. For the star HD 128620 ( $\alpha$ Cen A), the photometric determination of $T_{\text {eff }}$ was not performed (see discussion in Sect. 3.4).

References. ${ }^{1}$ Crawford (1975); Fabregat \& Reglero (1990); Ferro et al. (1990); Gronbech \& Olsen (1976, 1977); Olsen (1977, 1983, 1993, 1994a,b); Olsen \& Perry (1984); Perry et al. (1987); Reglero \& Fabregat (1991); Schuster \& Nissen (1988); Twarog (1980).

Figure 2 shows two examples of synthetic spectra of the $\mathrm{C}_{2}$ molecular band regions around $\lambda 5128$ and $\lambda 5165$, and of the $\mathrm{C}$ atomic lines at $\lambda 5052.2$ and $\lambda 5380.3$ for the sunlight spectrum reflected by Ganymede (second observation run) and for the metal-rich star HD 160691. The spectral synthesis was first applied to the Ganymede spectra of both runs, then the $g f$ values of some atomic and molecular lines were revised when needed, and finally the synthesis was applied to the other stars, treated according to their observation runs. For each case, the best fit was obtained through the minimisation of the rms between observed and synthetic spectra.

In order to estimate the uncertainties in the $[\mathrm{C} / \mathrm{Fe}]$ abundance determination, we performed a spectral synthesis of the most prominent molecular band used ( 25165$)$ adopting model atmospheres perturbed by the errors estimated for the atmospheric parameters. This procedure resulted in: \pm 0.03 dex due to the error in $T_{\mathrm{eff}}^{\mathrm{exc}} ; \pm 0.01$ dex due to the error in $[\mathrm{Fe} / \mathrm{H}] ; \pm 0.02$ dex due to the error in $\xi$; and \pm 0.03 dex due to the error in $\log g_{\text {ion. }}$. The uncertainties related to errors in $V_{\text {broad }}\left(\sim 1.0 \mathrm{~km} \mathrm{~s}^{-1}\right.$ or smaller $)$ and in the limb darkening coefficient are negligible. The quadratic sum of the individual contributions (also including a global error of 0.05 dex estimated based on the rms minimisation of the solar spectrum) yields a total uncertainty $\sigma([\mathrm{C} / \mathrm{Fe}])=0.07$ dex.

\section{Evolutionary, kinematic, and orbital parameters}

\subsection{Mass and age determination}

The evolutionary parameters mass and age were obtained by interpolation in the Yonsei-Yale $\left(Y^{2}\right)$ evolutionary tracks and isochrones (Yi et al. 2001; Kim et al. 2002) drawn on the HR diagram and computed for different values of metallicity.

The luminosity used in the diagrams were calculated with parallaxes taken from the new reduction of the Hipparcos data (van Leeuwen 2007), bolometric corrections (BC) from Flower (1996), and an absolute bolometric magnitude for the Sun $M_{\text {bol }}^{\odot}=4.75$ for $M_{\mathrm{v}}^{\odot}=4.82$. We estimated, for these nearby stars with precise parallaxes, a mean uncertainty of $0.01 \mathrm{dex}$ in $\log \left(L / L_{\odot}\right)$.

The effective temperature is a weighted mean ( $\left.T_{\text {eff }}^{\text {mean }}\right)$ of the excitation, photometric, and $\mathrm{H} \alpha$ temperatures. The weights are given by $1 / \sigma^{2}$ for $\sigma\left(T_{\mathrm{eff}}^{\mathrm{exc}}\right)=30 \mathrm{~K}, \sigma\left(T_{\mathrm{eff}}^{\mathrm{phot}}\right)=40 \mathrm{~K}$, and $\sigma\left(T_{\mathrm{eff}}^{\mathrm{H} \alpha}\right)=50 \mathrm{~K}$, obtained as described in Sects. 3.2 and 3.4. The uncertainty of the weighted mean is $22 \mathrm{~K}$, calculated using these three values of $\sigma$. On the other hand, the standard deviations of the three values of temperature around the weighted mean is $29 \mathrm{~K}$. Therefore, we conclude that our estimates of effective temperature based on the three indicators agree with each other very well (see the comparison in Fig. 3) and that the mean value has a mean internal error $\sigma\left(T_{\text {eff }}^{\text {mean }}\right)=30 \mathrm{~K}$.

Porto de Mello et al. (2008) determined the effective temperature of $\alpha$ Cen A (HD 128620) and B also using the excitation, photometric, and $\mathrm{H} \alpha$ approaches. They found a good agreement for $\alpha$ Cen A, a solar temperature star. However, for $\alpha$ Cen B $\left(T_{\text {eff }} \sim 5200 \mathrm{~K}\right)$, the excitation effective temperature is about $100-150 \mathrm{~K}$ higher than the photometric and the $\mathrm{H} \alpha$ counterparts, which the authors attributed to non-LTE effects. Although the agreement for the coolest and hottest stars in our sample is not that good, especially in the comparison of excitation and $\mathrm{H} \alpha$ temperatures, Fig. 3 does not show any systematic difference 

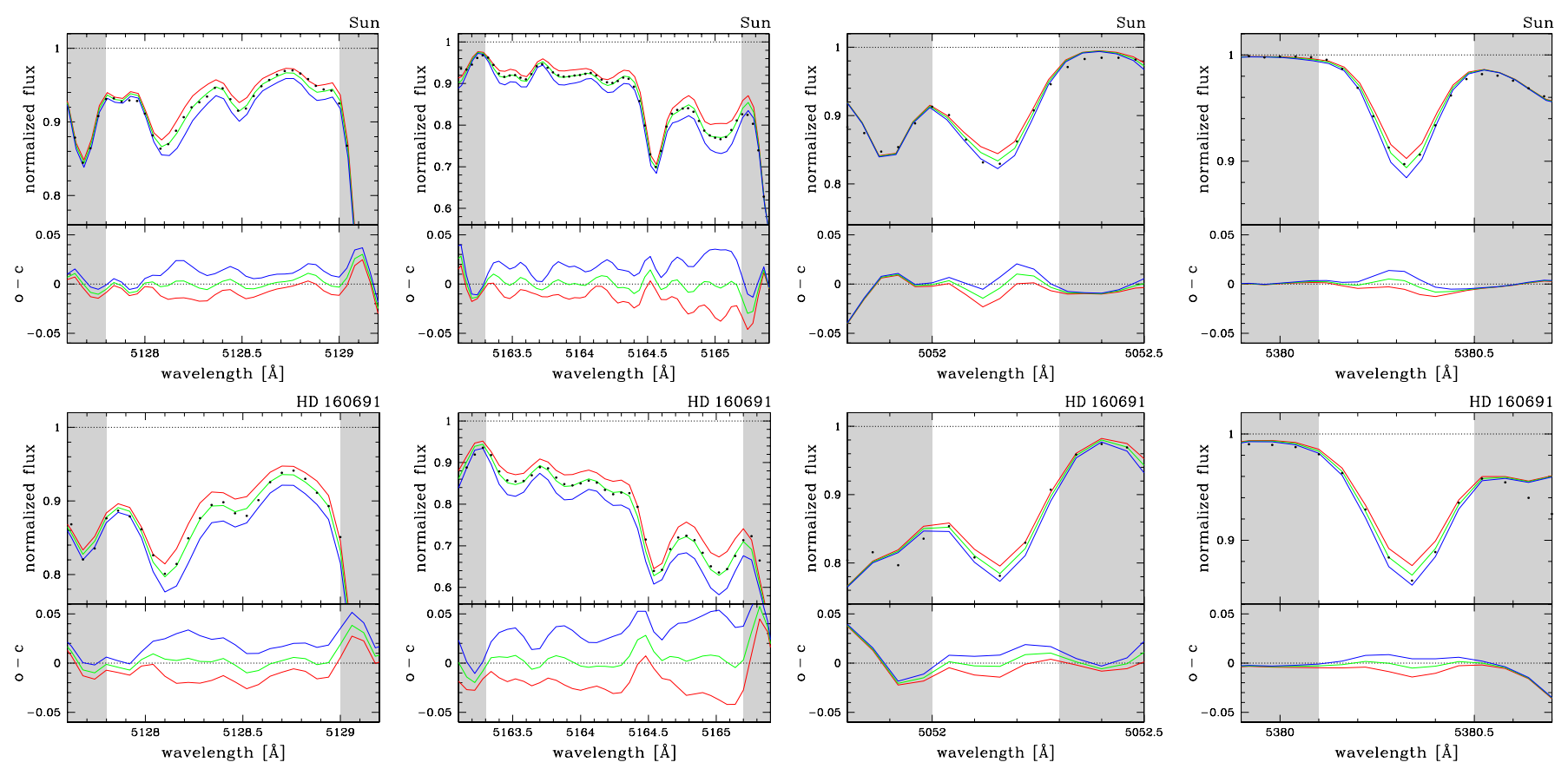

Fig. 2. Spectral synthesis of the $\mathrm{C}_{2}$ molecular band regions ( $\lambda 5128$ and $\left.\lambda 5165\right)$ and of the $\mathrm{C}$ atomic lines ( $\lambda 5052.2$ and $\left.\lambda 5380.3\right)$ for Ganymede of the second observation run (top panels) and for one metal-rich star of the first run, HD 160691 (bottom panels). The solid lines represent three models with different values of $[\mathrm{C} / \mathrm{Fe}]:-0.05$ (red), 0.0 (green), and +0.05 dex (blue). The differences between observed (dots) and computed spectra $(\mathrm{o}-\mathrm{c})$ are also shown in the bottom of each panel.

among the three indicators, and the differences are nonetheless within $2 \sigma$ for all the $T_{\text {eff }}$ range (only HD 115383 has $T_{\text {eff }}^{\text {exc }}$ larger than $T_{\mathrm{eff}}^{\mathrm{H} \alpha}$ by $\left.3 \sigma\right)$. This confirms that 1D LTE model atmospheres may adequately represent solar-type stars, at least in a differential analysis relative to the Sun.

We remind that the temperature used in our abundance analyses (based on the equivalent widths or synthesis of spectral features) was the excitation effective temperature, which better characterises the temperature radial profile in the stellar photosphere and the formation of absorption lines in the emergent spectrum. On the other hand, to better represent the luminosity of a star and to account for any possible effect due to small deviations from LTE, we adopted the weighted mean of the three temperature indicators.

The stars were grouped according to their values of metallicity (12 groups from $[\mathrm{Fe} / \mathrm{H}]=-0.32$ to $+0.32 \mathrm{dex}$ ) and then their masses and ages were computed using evolutionary tracks and isochrones for each stellar group. The difference in metallicity between each star and its respective HR diagram is not greater than 0.02 dex. A few examples for some metallicities are shown in Fig. 4. To reproduce the Sun's position in the diagrams, adopting $T_{\text {eff }}=5777 \mathrm{~K}$ and age $=4.53 \mathrm{~Gy}$ (Guenther \& Demarque 1997), the evolutionary tracks and isochrones were displaced in $\log \left(T_{\text {eff }}\right)$ and $\log \left(L / L_{\odot}\right)$ by $0.001628\left(\sim 22 \mathrm{~K}\right.$ in $\left.T_{\text {eff }}\right)$ and 0.011 , respectively. These values are, at any rate, of the same order or smaller than the uncertainties on these parameters.

As an independent check, we calculated the evolutionary surface gravity using the values of mass and effective temperature obtained, which we called $g_{\text {evol }}$, using the following equation:

$\log \frac{g_{\mathrm{evol}}}{g_{\odot}}=\log \frac{M}{M_{\odot}}+4 \log \frac{T_{\mathrm{eff}}^{\text {mean }}}{T_{\mathrm{eff}}^{\odot}}+0.4\left(M_{\mathrm{bol}}-M_{\mathrm{bol}}^{\odot}\right)$

where $M_{\text {bol }}$ is the absolute bolometric magnitude for the stars. The values of $\log g_{\text {evol }}$ are listed in Table 2 together with the ionisation surface gravity. They are in very good agreement, having a dispersion of only 0.09 dex, smaller than the uncertainty of 0.13 dex estimated for $\log g_{\text {ion }}$.

\subsection{Galactic velocities, distance, and eccentricity}

The kinematic properties of our sample were investigated by computing the Galactic velocity components $U_{\mathrm{LSR}}, V_{\mathrm{LSR}}$, and $W_{\text {LSR }}$ (see Fig. 5) with respect to the Local Standard of Rest (LSR). We developed a code that uses equations of Johnson \& Soderblom (1987), parallaxes and proper motions both from the new reduction of the Hipparcos data, and radial velocities from Holmberg et al. (2007), Torres et al. (2006) for HD 114613, and Santos et al. (2004) for HD 160691. For the Sun, the adopted values of $U_{\mathrm{LSR}}, V_{\mathrm{LSR}}$, and $W_{\mathrm{LSR}}$ are $10.0,5.3$, and $7.2 \mathrm{~km} \mathrm{~s}^{-1}$, respectively (Dehnen \& Binney 1998).

The mean orbital distance from the Galactic centre $\left(R_{\mathrm{m}}\right)$ and the orbital eccentricity $(e)$ were also considered in our analysis (see Fig. 5), where $e=\left(R_{\mathrm{a}}-R_{\mathrm{p}}\right) /\left(R_{\mathrm{a}}+R_{\mathrm{p}}\right)$ and $R_{\mathrm{m}}=\left(R_{\mathrm{a}}+R_{\mathrm{p}}\right) / 2$ were computed using the perigalactic $\left(R_{\mathrm{p}}\right)$ and the apogalactic $\left(R_{\mathrm{a}}\right)$ orbital distances from the Geneva-Copenhagen survey (Holmberg et al. 2009). For the Sun, the adopted values are $e=0.06$ and $R_{\mathrm{m}}=8 \mathrm{kpc}$.

\section{Tree clustering analysis}

We looked for statistically significant abundance groups in our sample using a hierarchical clustering analysis. To avoid missing abundance values, the analysis uses only those elements having abundances measured for all stars and was applied to the $[\mathrm{X} / \mathrm{H}]$ abundance space.

We used the complete linkage method for the hierarchical clustering (Everitt et al. 2001) and euclidean distances as measures of dissimilarities in this abundance space. A hierarchical clustering algorithm works by joining similar objects in a hierarchical structure. Initially, each object is assigned to its own 
R. da Silva et al.: Chemo-chronological analysis of solar-type stars

Table 5. Evolutionary, kinematic, and orbital parameters, separating the stars according to the classification of Sect. 5.

\begin{tabular}{|c|c|c|c|c|c|c|c|c|c|c|c|c|}
\hline & $\begin{array}{c}T_{\text {eff }}^{\text {mean }} \\
{[\mathrm{K}]}\end{array}$ & {$[\mathrm{Fe} / \mathrm{H}]$} & $\log L / L_{\odot}$ & $M / M_{\odot}$ & $\begin{array}{l}\text { age } \\
{[\text { Gyr] }}\end{array}$ & $\begin{array}{c}V_{\text {broad }} \\
{\left[\mathrm{km} \mathrm{s}^{-1}\right]}\end{array}$ & $F_{\mathrm{H} \alpha}^{\prime}$ & $\begin{array}{c}U_{\mathrm{LSR}} \\
{\left[\mathrm{km} \mathrm{s}^{-1}\right]}\end{array}$ & $\begin{array}{c}V_{\mathrm{LSR}} \\
{\left[\mathrm{km} \mathrm{s}^{-1}\right]}\end{array}$ & $\begin{array}{c}W_{\mathrm{LSR}} \\
{\left[\mathrm{km} \mathrm{s}^{-1}\right]}\end{array}$ & $\begin{array}{c}R_{\mathrm{m}} \\
{[\mathrm{kpc}]}\end{array}$ & $e$ \\
\hline Sun & 5777 & 0.00 & 0.00 & 1.00 & 4.53 & 1.8 & 3.4 & 10.0 & 5.3 & 7.2 & 8.00 & 0.06 \\
\hline \multicolumn{13}{|c|}{ metal-poor stars: } \\
\hline HD 20807 & 5874 & -0.22 & -0.02 & 0.96 & 4.2 & $<2$ & 3.6 & -59.3 & -41.2 & 24.2 & 7.06 & 0.24 \\
\hline HD 33021 & 5778 & -0.20 & 0.35 & 0.98 & 10.1 & 4.1 & 3.8 & 48.2 & -29.3 & -12.2 & 7.30 & 0.19 \\
\hline HD 53705 & 5815 & -0.22 & 0.14 & 0.93 & 10.1 & 4.0 & 1.9 & -43.4 & -68.9 & -12.9 & 6.33 & 0.31 \\
\hline HD 102365 & 5664 & -0.28 & -0.09 & 0.86 & 10.1 & $<2$ & 2.1 & -49.4 & -33.5 & 13.1 & 7.18 & 0.20 \\
\hline HD 196761 & 5456 & -0.32 & -0.27 & 0.80 & 10.8 & 3.3 & 4.9 & -49.2 & 25.3 & 12.1 & 9.02 & 0.18 \\
\hline HD 189567 & 5703 & -0.27 & 0.00 & 0.87 & 11.9 & 4.4 & 2.9 & -60.6 & -26.5 & -41.4 & 7.49 & 0.21 \\
\hline \multicolumn{13}{|c|}{ intermediate abundance (under solar) stars: } \\
\hline HD 26491 & 5805 & -0.09 & 0.13 & 0.97 & 8.5 & 3.5 & 2.1 & -30.2 & -20.8 & -8.0 & 7.42 & 0.12 \\
\hline HD 84117 & 6122 & -0.06 & 0.29 & 1.12 & 3.9 & 5.9 & 0.0 & -30.2 & -20.8 & 14.1 & 7.45 & 0.12 \\
\hline HD 117176 & 5567 & -0.04 & 0.47 & 1.08 & 8.2 & $<2$ & 1.5 & 23.1 & -46.2 & 3.0 & 6.75 & 0.20 \\
\hline HD 181321 & 5832 & -0.06 & -0.12 & $\sim 1.02$ & 0.2 & 12.5 & 14.2 & -2.9 & -1.9 & 3.1 & 7.97 & 0.01 \\
\hline \multicolumn{13}{|c|}{ intermediate abundance (over solar) stars: } \\
\hline HD 39587 & 5981 & 0.00 & 0.03 & $\sim 1.10$ & 0.3 & 9.0 & 10.3 & 24.0 & 7.3 & -0.1 & 8.29 & 0.08 \\
\hline HD 43834 & 5636 & 0.11 & -0.08 & 0.99 & 3.9 & $<2$ & 2.8 & 29.0 & -24.8 & -4.6 & 7.31 & 0.13 \\
\hline HD 50806 & 5621 & 0.02 & 0.35 & 1.02 & 9.9 & 2.0 & 2.8 & 27.8 & -89.5 & 0.9 & 5.84 & 0.39 \\
\hline HD 114613 & 5701 & 0.15 & 0.62 & 1.26 & 5.1 & $<2$ & 1.7 & -28.1 & -5.7 & 9.4 & - & - \\
\hline HD 115617 & 5595 & 0.00 & -0.09 & 0.94 & 6.8 & $<2$ & 4.2 & -13.8 & -42.2 & -24.3 & 6.85 & 0.17 \\
\hline HD 141004 & 5924 & 0.03 & 0.32 & 1.10 & 6.3 & $<2$ & 1.6 & -39.7 & -19.4 & -32.3 & 7.55 & 0.14 \\
\hline HD 146233 & 5816 & 0.05 & 0.02 & 1.03 & 3.4 & $<2$ & 2.7 & 36.8 & -9.0 & -15.3 & 7.79 & 0.12 \\
\hline HD 147513 & 5888 & 0.04 & -0.02 & $\sim 1.05$ & 0.3 & $<2$ & 7.0 & 23.2 & 4.4 & 5.8 & 8.15 & 0.07 \\
\hline HD 177565 & 5644 & 0.08 & -0.06 & 1.00 & 4.2 & 2.3 & 3.8 & 67.3 & -27.8 & -9.0 & 7.41 & 0.23 \\
\hline HD 188376 & 5493 & 0.00 & 0.90 & 1.53 & 2.7 & $<2$ & 0.5 & -23.6 & 12.5 & -2.3 & 8.49 & 0.09 \\
\hline \multicolumn{13}{|c|}{ metal-rich stars: } \\
\hline HD 1835 & 5863 & 0.21 & 0.00 & $\sim 1.15$ & 0.6 & 5.9 & 7.7 & -26.5 & -9.9 & 7.4 & 7.74 & 0.09 \\
\hline HD 112164 & 6014 & 0.32 & 0.76 & $1.40 *$ & $3.5^{*}$ & 4.6 & 1.4 & -1.4 & -60.4 & -21.9 & 6.43 & 0.24 \\
\hline HD 115383 & 6075 & 0.23 & 0.32 & 1.22 & 2.9 & 7.0 & 8.0 & -28.1 & 6.7 & -10.8 & 8.27 & 0.09 \\
\hline HD 128620 & 5847 & 0.23 & 0.17 & 1.11 & 4.4 & $<2$ & 4.7 & -21.7 & 8.3 & 19.9 & 8.31 & 0.07 \\
\hline HD 160691 & 5747 & 0.27 & 0.26 & $1.12 *$ & $6.2 *$ & $<2$ & 2.4 & -3.7 & -3.4 & 3.4 & - & - \\
\hline
\end{tabular}

Notes. The $\mathrm{H} \alpha$ fluxes $\left(F_{\mathrm{H} \alpha}^{\prime}\right)$, from Lyra \& Porto de Mello (2005), are given in units of $10^{5} \mathrm{erg} \mathrm{cm}^{-2} \mathrm{~s}^{-1}$. The mean distance from the Galactic centre $\left(R_{\mathrm{m}}\right)$ and the orbital eccentricity $(e)$ are from Holmberg et al. (2009). The asterisk $(*)$ indicates stars adjacent to superposed evolutionary tracks and isochrones on the HR diagram.

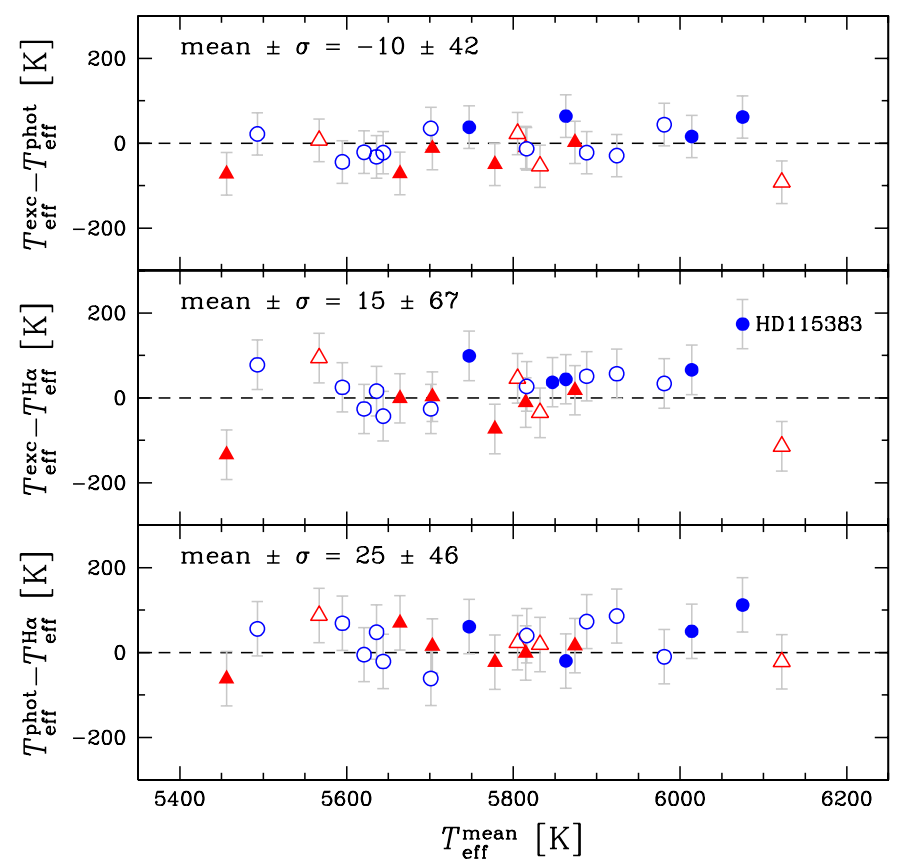

Fig. 3. Comparisons of the three effective temperature indicators: the excitation vs. photometric (top panel), excitation vs. $\mathrm{H} \alpha$ (middle panel), and photometric vs. $\mathrm{H} \alpha$ (bottom panel) temperatures. The symbols represent the stellar groups defined in Sect. 5 (see Fig. 6). cluster. The algorithm proceeds iteratively, joining the two most similar clusters in each pass until there is just a single cluster. The resulting hierarchy of clusters for our data is shown in Fig. 6 (upper panel) as a dendrogram. In this plot, the most similar objects are linked together in the bottom forming clusters, which are then iteratively linked together in pairs by similarity. The vertical axis in a dendrogram measures the dissimilarity between each individual or cluster. Since we used euclidean distances in the $[\mathrm{X} / \mathrm{H}]$ abundance space, the units of this axis is dex, although it measures the total dissimilarity in this abundance space and not in a single variable.

Clusters can be defined by specifying a reasonable total dissimilarity value for pruning the dendrogram. There is no unambiguous or optimal way for defining this pruning value, especially because the clusters found depend on the clustering method and cluster shapes. Since our sample is quite small, we arbitrarily decided to prune our dendrogram at the total dissimilarity of 0.9 dex, which is shown in Fig. 6 by the dashed red line. This pruning value was chosen in order to have four more or less equally populated clusters. Considering the size of our sample, less than four clusters would simply limit our discussion to poor against rich stars, while a larger number of clusters would make such an analysis meaningless.

The average $\langle[\mathrm{X} / \mathrm{H}]\rangle$ behaviour of the four clusters for each element considered in this analysis is shown in the middle panel of Fig. 6. Two of these clusters have over-solar abundances, with averages +0.26 and +0.06 dex for all the elements, whereas 

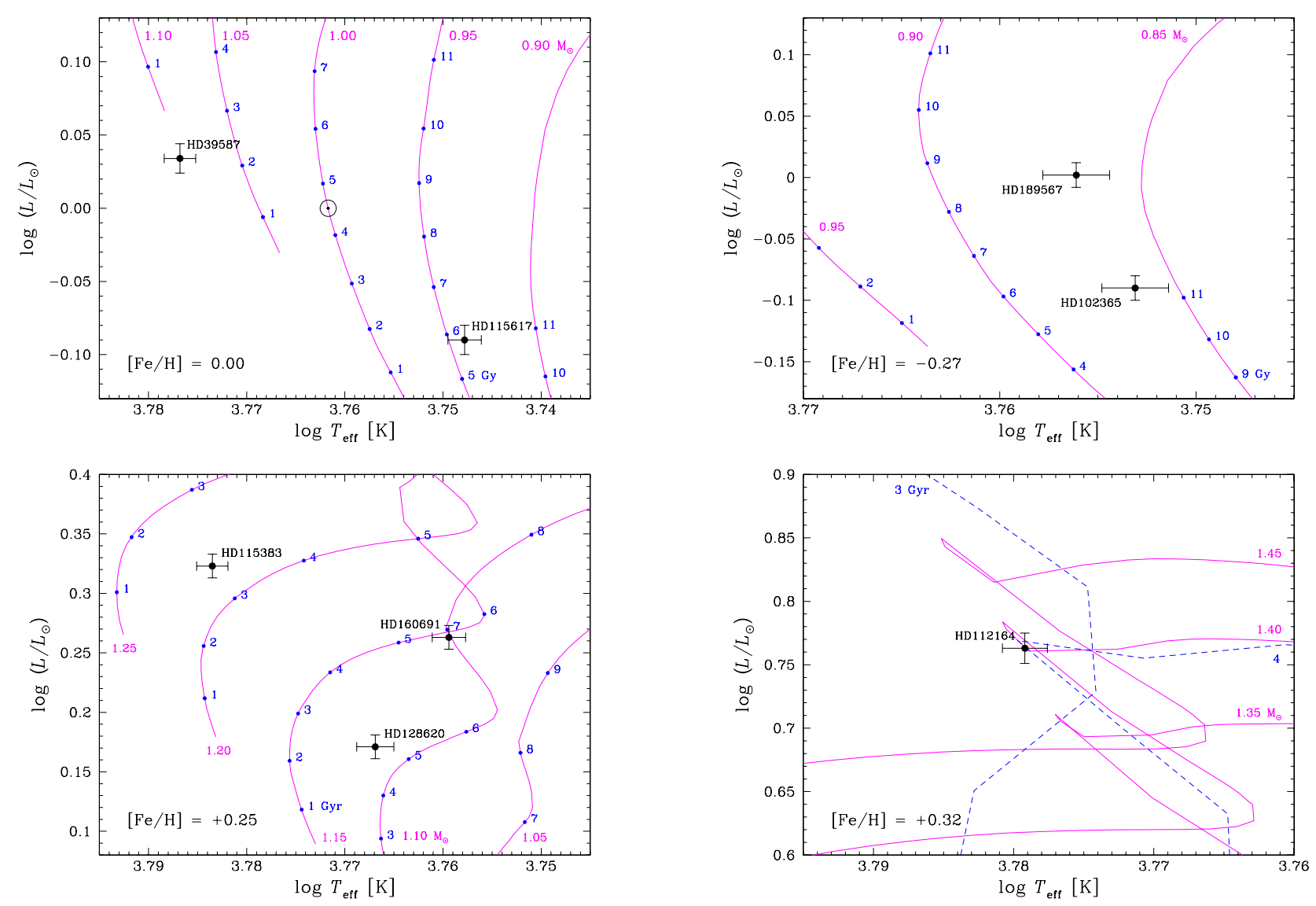

Fig. 4. Evolutionary tracks (magenta solid lines) and isochrones (blue dots and dashed lines) from Yonsei-Yale, calculated for different values of metallicity, showing how to derive the stellar masses and ages. The ages in Gyr are indicated alongside the evolutionary tracks, for which the masses are indicated in $M_{\odot}$. The Sun's position for $T_{\mathrm{eff}}=5777 \mathrm{~K}$ is also shown in the solar metallicity panel (top-left corner).
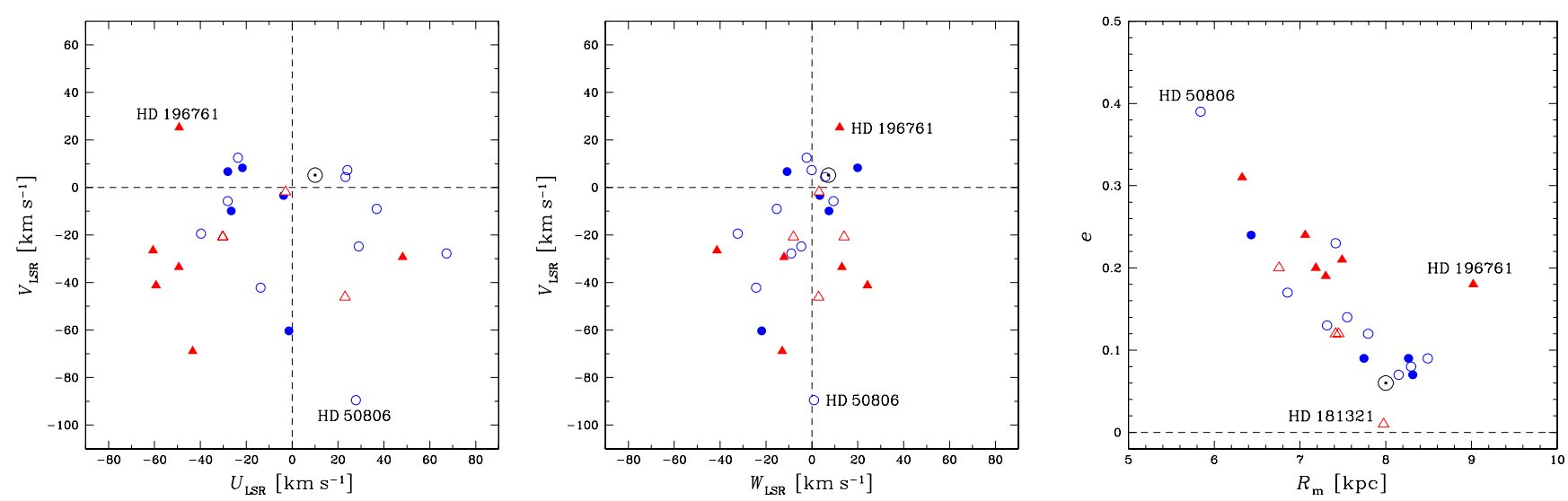

Fig. 5. Galactic velocity components (left and middle panels) and orbital eccentricity as a function of the mean orbital distance from the Galactic centre (right panel) for the sample stars and the Sun. The symbols represent the stellar groups defined in Sect. 5.

the two others have under-solar abundance values, with averages -0.06 and -0.24 dex. We can observe in the figure that the elemental abundance patterns of the metal-poor and metal-rich groups are distinct between each other. In particular, it seems to exit a chemical distinction even between the two intermediate groups: the under-solar intermediate group has an abundance pattern that roughly follows the element by element pattern of the metal-poor group, whereas the pattern of the over-solar intermediate group resembles the scaled-solar mixture.
The clustering analysis we have presented was tentatively based on biological ideas of evolving species, in the sense that the material the stars came from is continuously changing. For this reason we made use of $[\mathrm{X} / \mathrm{H}]$ abundances ratios instead of $[\mathrm{X} / \mathrm{Fe}]$. We implicitly need the time evolution that $[\mathrm{X} / \mathrm{H}]$ has, because we want a time hierarchy in the output groups. A similar analysis in the $[\mathrm{X} / \mathrm{Fe}]$ space can still show groups, but the hierarchical relation between these groups in a dendrogram will not necessarily show evolutionary trends, because this 

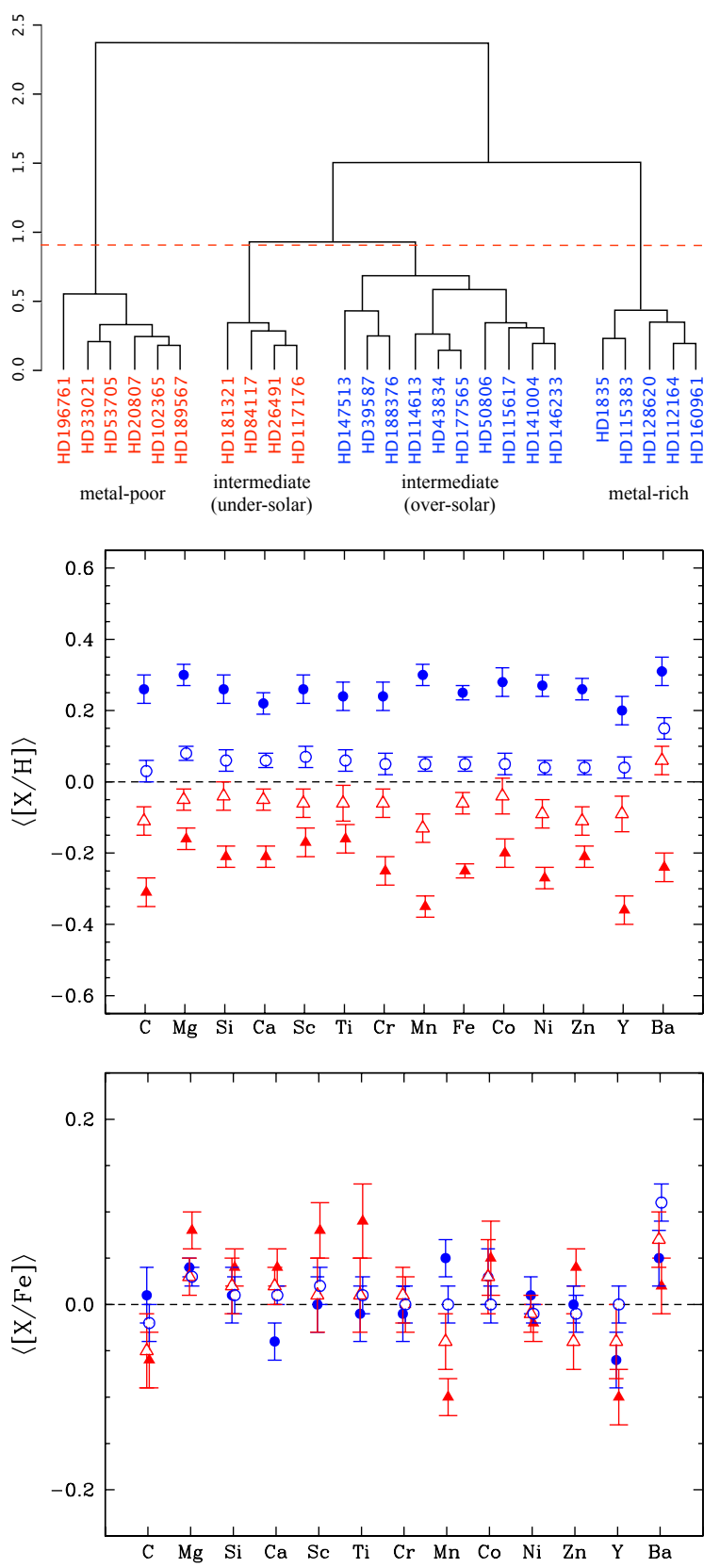

Fig. 6. Dendrogram (upper panel) and mean abundance ratios $\langle[\mathrm{X} / \mathrm{H}]\rangle$ (middle panel) and $\langle[\mathrm{X} / \mathrm{Fe}]\rangle$ (bottom panel) for the four stellar groups from the tree clustering analysis (Sect. 5). They are classified as metalpoor $(\mathbf{\Delta})$, intermediate abundance (under solar $\Delta$ or over solar $\circ$ ), or metal-rich $(\bullet)$ stars, and the same symbolism is adopted all over the paper. The dashed red line on the dendrogram represents a dissimilarity number of 0.9 dex.

variable is only indirectly linked with time. Notwithstanding, we have checked this, but the output groups show no meaningful interpretation in terms of chemical evolution or abundance ratio groups. The outcome could be different if the sample were larger, but this needs to be verified with another sample, what is beyond the scope of this paper. Nevertheless, we included in Fig. 6 (bottom panel) the average $\langle[\mathrm{X} / \mathrm{Fe}]\rangle$ behaviour of the stars clustered in the $[\mathrm{X} / \mathrm{H}]$ parameter space. The small variation of $\langle[\mathrm{X} / \mathrm{Fe}]\rangle$ with respect to the solar values reinforces our point above: that for this specific small sample, the $[\mathrm{X} / \mathrm{Fe}]$ parameter space is dynamically very narrow and does not favour a cluster analysis.

\section{Results and discussion}

Table 5 lists the evolutionary (mass and age), kinematic ( $U_{\mathrm{LSR}}$, $V_{\mathrm{LSR}}$, and $W_{\mathrm{LSR}}$ velocities), and orbital (mean orbital distance from the Galactic centre and orbital eccentricity) parameters computed for the programme stars. They are grouped following the tree clustering analysis performed in Sect. 5.

The uncertainties in the mass and age determination may vary widely depending on the stellar position in the HR diagram. We made an estimate of these errors for a few representative stars in our sample (cool and hot dwarfs and subgiants). We took into account the errors estimated for $\log \left(L / L_{\odot}\right), \log \left(T_{\text {eff }}\right)$, and also $[\mathrm{Fe} / \mathrm{H}]$ considering that the evolutionary tracks and isochrones are metallicity dependent. We found that the uncertainties in mass stand between 0.02 and $0.08 M_{\odot}$, whereas those in age vary from about $0.5 \mathrm{Gyr}$ (or smaller) for evolved stars up to about $2.5 \mathrm{Gyr}$ for cool main-sequence stars.

For HD 1835, HD 39587, HD 147513, and HD 181321 we determined an approximative value for their masses and an upper limit for their ages given their position in the HR diagram (close to the Zero Age Main Sequence). Indeed, these are very young stars: one of them, HD 1835, is likely a member of the Hyades star cluster ( $600 \mathrm{Myr})$ according to López-Santiago et al. (2010); two others, HD 39587 (Soderblom \& Mayor 1993; Fuhrmann 2004) and HD 147513 (Soderblom \& Mayor 1993; Montes et al. 2001) belong to the Ursa Major moving group of 300 Myr (see also Castro et al. 1999); and HD 181321 is a member of the Castor moving group ( $200 \mathrm{Myr})$ according to Montes et al. (2001). For these four stars, we adopted the ages of their respective moving group in our study.

The stars HD 112164 and HD 160691, indicated by asterisks (*) in Table 5, are located in a region of the HR diagram where successive evolutionary tracks and isochrones are superposed (see example in Fig. 4). Therefore, their mass and age determination may yield larger uncertainties: $\lesssim 0.12 M_{\odot}$ and $\lesssim 0.7 \mathrm{Gyr}$ for HD 112164, and $\lesssim 0.04 M_{\odot}$ and $\lesssim 1.0 \mathrm{Gyr}$ for HD 160691.

The $U_{\mathrm{LSR}}, V_{\mathrm{LSR}}$, and $W_{\mathrm{LSR}}$ velocities have a typical uncertainty of $0.3 \mathrm{~km} \mathrm{~s}^{-1}$ or smaller. An exception is the star HD 188376, for which the large errors $\left(2.5,1.1\right.$, and $1.8 \mathrm{~km} \mathrm{~s}^{-1}$, respectively) are due to a large uncertainty in its parallax.

The level of activity in the chromosphere of the stars, which is related to their age, was also investigated. The table lists the flux in the centre of the $\mathrm{H} \alpha$ line $\left(F_{\mathrm{H} \alpha}^{\prime}\right)$, computed by Lyra $\&$ Porto de Mello (2005) and used as a chromospheric activity indicator (the larger the value of $F_{\mathrm{H} \alpha}^{\prime}$, the higher the level of chromospheric activity). The uncertainty for this parameter is $0.5 \times 10^{5} \mathrm{erg} \mathrm{cm}^{-2} \mathrm{~s}^{-1}$.

We note that a few stars in our sample have at least one planetary companion detected. They are HD 102365, HD 115617 , HD 117176, HD 147513, and HD 160691 (see The Extrasolar Planets Encyclopaedia: http: //exoplanet . eu). Comparisons of the properties of stars with and without planets are frequently published. In the analysis performed in this paper, however, no peculiar information distinguishing the two populations has been found.

\subsection{Elemental abundances}

Table 10 lists the chemical abundances relative to iron $[\mathrm{X} / \mathrm{Fe}]$ obtained for the elements studied. For some elements of some stars, the abundance determination was not possible due to the poor quality or the weakness of their spectral lines (empty fields 
in the table). The carbon abundance ratios $[\mathrm{C} / \mathrm{Fe}]$ are shown in Table 6.

We also computed the mean abundance ratios $\langle[\mathrm{X} / \mathrm{Fe}]\rangle$ of the following groups of elements: i) two groups of light metals: $(\mathrm{Mg}, \mathrm{Si})$ and $(\mathrm{Ca}, \mathrm{Sc}, \mathrm{Ti})$; ii) two groups of the iron peak: (V, Cr, Co, Ni) and ( $\mathrm{Mn}, \mathrm{Cu})$; iii) light elements from the s-process: ( $\mathrm{Sr}, \mathrm{Y}, \mathrm{Zr}$ ), to which we refer as ls; and iv) heavy elements from the s-process: $(\mathrm{Ba}, \mathrm{Ce}, \mathrm{Nd})$, referred to as hs (see Table 7). They were grouped either because they have possibly the same nucleosynthetic origin or because they share a similar behaviour in the diagrams. The abundance ratio between heavy and light elements from the s-process, [hs/ls] = $[\mathrm{hs} / \mathrm{Fe}]-[\mathrm{ls} / \mathrm{Fe}]$, was also calculated.

Figure 7 shows diagrams with the abundance ratios of the programme stars for individual elements and nucleosynthetic groups. The uncertainties are listed in Tables 3 and 7. As for the individual elements, the estimated errors are compared to the dispersions around the mean for groups having at least two elements. For each group of each observation run (represented by the stars HD 146233 and HD 26491), the larger values were adopted to be the uncertainties in the grouped abundance ratios. In Fig. 7, the stars are represented by different symbols according to the tree clustering analysis performed in Sect. 5.

The star HD 1835 is enriched in $\mathrm{Ca}, \mathrm{Sr}$, and $\mathrm{Ba}$. The mean value of all s-process elements also suggests an over-solar abundance. Sm, the only r-process element analysed here, shows an under-solar abundance of -0.3 dex, but with a large error. As already mentioned, this is a very young star, a probable member of the Hyades star cluster of age $\sim 600 \mathrm{Myr}$, which is in agreement with its high level of chromospheric activity indicated by $F_{\mathrm{H} \alpha}^{\prime}$ in Table 5.

Two other very young stars are HD 39587 and HD 147513, both members of the kinematic Ursa Major group. They are clearly overabundant in the s-process elements, especially $\mathrm{Ba}$, and underabundant in $\mathrm{C}$, which is in agreement with the results of Porto de Mello \& da Silva (1997a) and Castro et al. (1999). These stars were proposed by Porto de Mello \& da Silva (1997a) to be barium stars, originated in a phenomenon in which the more massive component of a binary system evolves as a thermally pulsing asymptotic giant branch (TP-AGB) star and the material produced in the He-burning envelope, enriched in s-process elements, is dredged-up to the surface and then accreted by its companion by wind mass transfer. The initially more massive star is now a white dwarf whereas the companion has become the primary barium star. At present, HD 39587 is a single-lined spectroscopic and astrometric binary, with a low mass companion of $0.15 M_{\odot}$ (König et al. 2002), and HD 147513 has a common proper motion companion, a DA2 white dwarf, at an angular separation of 345" (Holberg et al. 2002). The barium-star scenario was not supported by Castro et al. (1999), who proposed that the two stars simply have usual Ba abundances for their age and that probably all the Ursa Major group members are Ba-enriched, either due to a primordial origin or because they are young (see discussion in Sect. 6.4).

HD 181321 and HD 188376 are two other Ba-rich stars. HD 188376 is the most evolved and massive star analysed here, clearly in the evolutionary stage of a subgiant. The other star, HD 181321, is the youngest and has the highest level of chromospheric activity in our sample. Indeed, our determination for $V_{\text {broad }}$ is $12.5 \mathrm{~km} \mathrm{~s}^{-1}$, indicating a fast-rotating star. It has solar atmospheric parameters, excepting a high value of microturbulence velocity $\left(\xi=2.3 \mathrm{~km} \mathrm{~s}^{-1}\right)$. The kinematic and orbital parameters are also very close to the solar values. In other words, this star has, on the one hand, about the same effective
Table 6. Carbon abundance ratios derived from the spectral synthesis of the two atomic lines $(\lambda 5052.2$ and $\lambda 5380.3)$ and the two $C_{2}$ band heads ( $\lambda 5128$ and $\lambda 5165)$ studied.

\begin{tabular}{lccccc}
\hline \hline \multirow{2}{*}{ Star } & {$[\mathrm{C} / \mathrm{Fe}]$} & {$[\mathrm{C} / \mathrm{Fe}]$} & {$[\mathrm{C} / \mathrm{Fe}]$} & {$[\mathrm{C} / \mathrm{Fe}]$} & {$[\mathrm{C} / \mathrm{Fe}]$} \\
\hline HD 1835 & $\lambda 5052$ & $\lambda 5380$ & $\lambda 5128$ & $\lambda 5165$ & \\
HD 20807 & 0.07 & 0.05 & 0.02 & 0.04 & 0.05 \\
HD 26491 & 0.01 & 0.05 & -0.11 & 0.05 & 0.01 \\
HD 33021 & -0.05 & -0.04 & -0.06 & -0.04 & -0.02 \\
HD 39587 & -0.07 & -0.07 & -0.12 & -0.19 & -0.11 \\
HD 43834 & -0.04 & 0.01 & 0.01 & 0.02 & -0.10 \\
HD 50806 & 0.21 & 0.13 & 0.08 & 0.08 & 0.00 \\
HD 53705 & -0.07 & -0.04 & -0.14 & -0.07 & -0.08 \\
HD 84117 & 0.09 & 0.07 & -0.06 & -0.06 & 0.01 \\
HD 102365 & 0.07 & 0.12 & -0.08 & 0.02 & 0.03 \\
HD 112164 & -0.01 & -0.01 & 0.00 & 0.02 & 0.00 \\
HD 114613 & -0.01 & -0.02 & 0.03 & 0.00 & 0.00 \\
HD 115383 & -0.12 & -0.09 & -0.05 & -0.05 & -0.08 \\
HD 115617 & -0.11 & -0.04 & 0.00 & 0.04 & -0.03 \\
HD 117176 & -0.06 & -0.04 & -0.06 & -0.04 & -0.05 \\
HD 128620 & 0.01 & 0.04 & 0.03 & 0.07 & 0.04 \\
HD 141004 & -0.03 & -0.03 & 0.00 & 0.07 & 0.00 \\
HD 146233 & -0.04 & -0.05 & -0.07 & 0.01 & -0.04 \\
HD 147513 & -0.15 & -0.11 & -0.18 & -0.10 & -0.14 \\
HD 160691 & 0.01 & -0.01 & 0.02 & 0.06 & 0.02 \\
HD 177565 & 0.05 & 0.10 & 0.02 & 0.03 & 0.05 \\
HD 181321 & - & -0.10 & -0.15 & -0.15 & -0.13 \\
HD 188376 & -0.16 & -0.06 & -0.02 & -0.05 & -0.07 \\
HD 189567 & 0.00 & -0.05 & -0.15 & -0.10 & -0.08 \\
HD 196761 & - & -0.13 & -0.20 & -0.13 & -0.15 \\
\hline
\end{tabular}

Notes. Column 6 shows the final values adopted.

Table 7. Groups of chemical elements and uncertainties in the mean abundance ratios $\langle[\mathrm{X} / \mathrm{Fe}]\rangle$.

\begin{tabular}{llcccc}
\hline \hline \multirow{2}{*}{ Nucleosynthetic group } & \multicolumn{2}{c}{$\mathrm{HD} 146233$} & \multicolumn{2}{c}{$\mathrm{HD} 26491$} \\
& & $\sigma_{\text {est }}$ & $\sigma_{\text {disp }}$ & $\sigma_{\text {est }}$ & $\sigma_{\text {disp }}$ \\
\hline Light metals & $\mathrm{Mg}, \mathrm{Si}$ & 0.03 & 0.02 & 0.04 & 0.03 \\
Light metals & $\mathrm{Ca}, \mathrm{Sc}, \mathrm{Ti}$ & 0.03 & 0.04 & 0.05 & 0.01 \\
Iron peak & $\mathrm{V}, \mathrm{Cr}, \mathrm{Co}, \mathrm{Ni}$ & 0.03 & 0.04 & 0.02 & 0.03 \\
Iron peak & $\mathrm{Mn}, \mathrm{Cu}$ & 0.03 & 0.01 & 0.05 & 0.02 \\
Light s-process & $\mathrm{Sr}, \mathrm{Y}, \mathrm{Zr}$ & 0.04 & 0.09 & 0.05 & 0.01 \\
Heavy s-process & $\mathrm{Ba}, \mathrm{Ce}, \mathrm{Nd}$ & 0.06 & 0.06 & 0.06 & 0.07 \\
\hline
\end{tabular}

Notes. The estimated errors are compared to the dispersions around the mean. For each group, the larger value of each observation run was adopted.

temperature, metallicity, surface gravity, mass, Galactic orbit, and space velocities as the Sun. On the other hand, it is very young and significantly enriched in $\mathrm{Ba}$, strengthening the relation between Ba abundance and age (see Sect. 6.4).

The high microturbulence velocity of the star HD 181321 is probably prompted by the strengthened convection and turbulence in its upper photosphere, which is subjected to large nonthermal energy influxes from the chromosphere. The UV radiation excess from the chromosphere of an active star can scape to the photosphere and cause departures from LTE due to an ionisation imbalance. The induced overionisation is commonly manifested by differences either in excitation and photometric effective temperatures, or in ionisation and evolutionary surface gravities (see Porto de Mello et al. 2008; Ribas et al. 2010). For HD 181321, our determination of $T_{\mathrm{eff}}^{\mathrm{exc}}$ and $T_{\mathrm{eff}}^{\text {phot }}$ are in very good 
R. da Silva et al.: Chemo-chronological analysis of solar-type stars
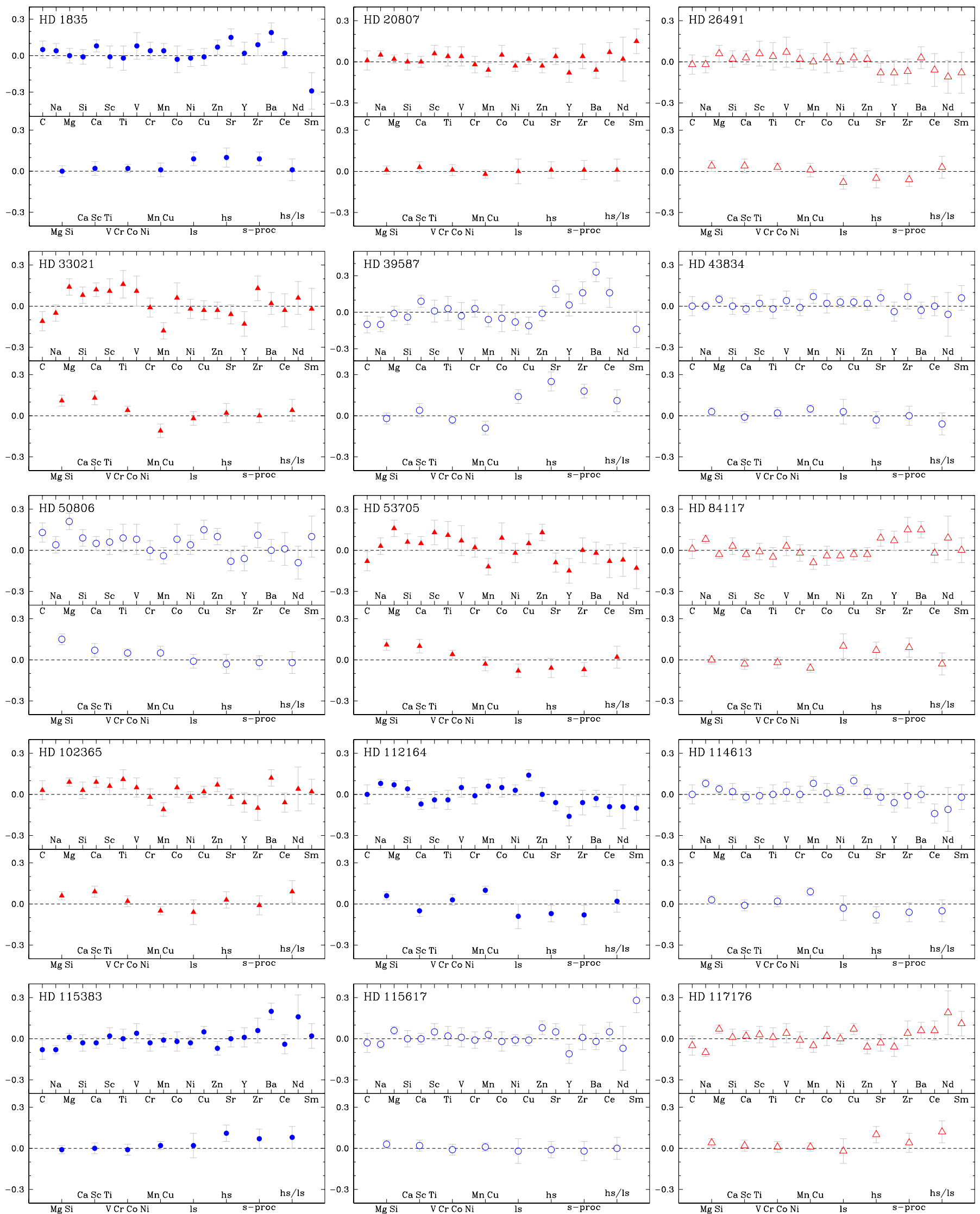

Fig. 7. Abundance ratios $[\mathrm{X} / \mathrm{Fe}]$ for individual elements (top of each panel) and $\langle[\mathrm{X} / \mathrm{Fe}]\rangle$ for the nucleosynthetic groups (bottom of each panel). The uncertainties adopted are those listed in Tables 3 and 7. The symbols represent the stellar classification defined in Sect. 5 (see Fig. 6). 

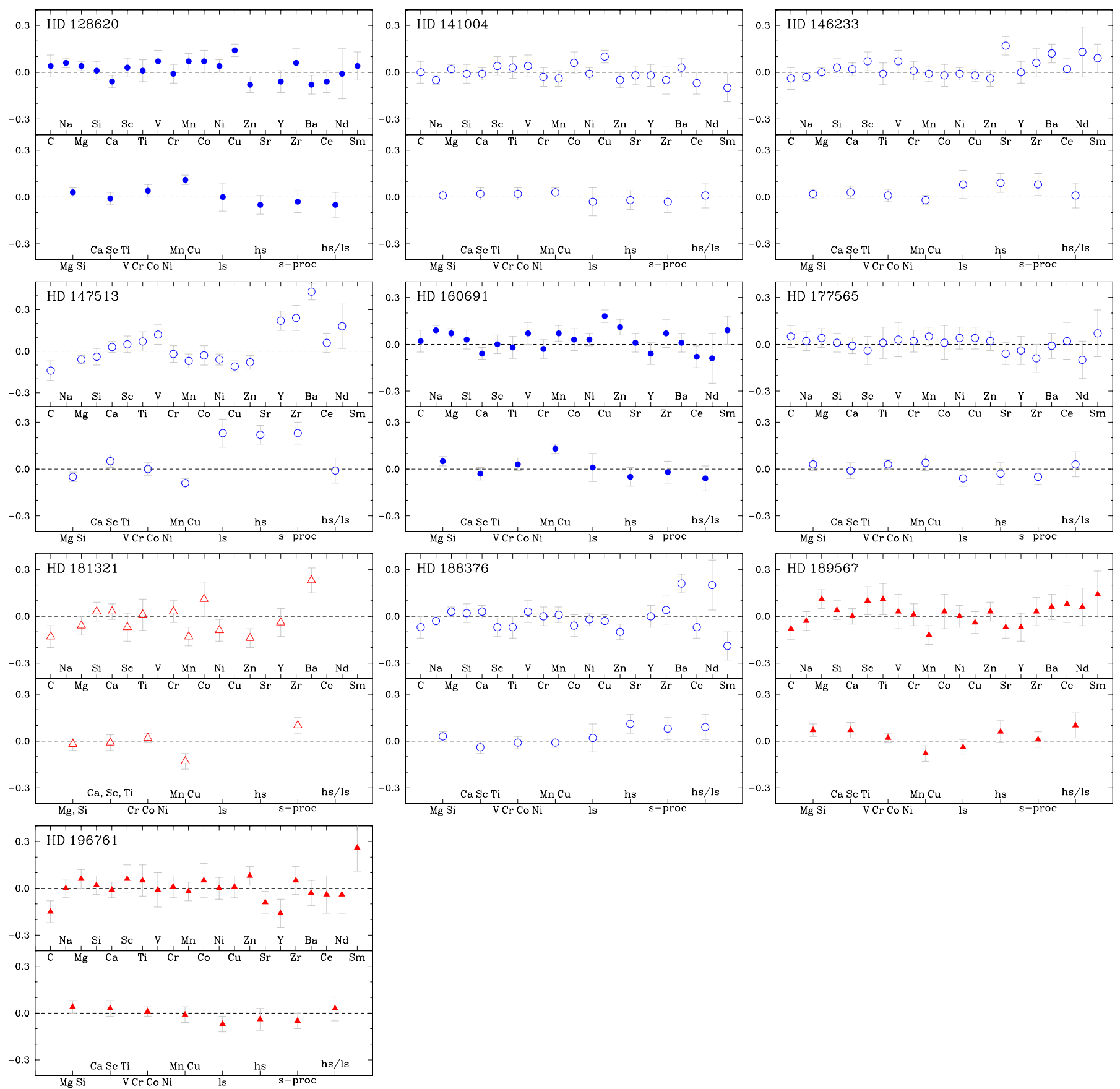

Fig. 7. continued.

agreement with each other. Therefore, only the difference in surface gravity and the large value of microturbulence velocity are possible signs that an overionisation is taking place in the photosphere of this active star. A full non-LTE analysis and a photospheric and chromospheric modelling would probably settle the issue, but this goes beyond the scope of this paper.

It is also very worthwhile to investigate the chemical abundances in stars that share similar values of age, metallicity, and Galactic orbit $\left(R_{\mathrm{m}}\right.$ and $e$ ). These subgroup of stars are supposed to share the same physical conditions of the Galaxy at the time and galactocentric position of their birth. An example of this includes the stars HD 43834, HD 84117, HD 141004, and HD 146233, which also have ages, metallicities, and Galactic orbits close to the solar values (they were all classified as intermediate abundance stars in the tree clustering analysis, one in slight underabundance and the three others in slight overabundance with respect to the Sun). In spite of this, only HD 43834 and HD 141004 show solar abundances, within the uncertainties, for all (or almost all) elements. HD 84117 is deficient in $\mathrm{Mn}$ and enriched in $\mathrm{Na}$ and in elements of the s-process (Sr, Y, Zr, and Ba). HD 146233, proposed by Porto de Mello \& da Silva (1997b) as the closest solar twin ever known at that time, is actually (as also proposed by these authors) enriched in some elements of the s-process ( $\mathrm{Sr}$ and $\mathrm{Ba}$ ) and possibly enriched in Sc, V, and Sm. Thus, possibly, the investigation of a larger sample of stars at a similar level of detail as done here could reveal that non-solar abundance ratios are present even for stars sharing the same place and time of birth. Whether this 
reflects intrinsic heterogeneities in their natal interstellar clouds (in principle a reasonable hypothesis since the elements reflecting non-solar ratios are related to different nucleosynthetic processes operating in different timescales) or else is evidence for considerable radial migration in the Galaxy is a question we plan to address in a subsequent work involving a larger sample.

Still concerning the relations involving the stellar groups from the clustering analysis and the kinematic and orbital parameters of our sample, we can see in Fig. 5 that the group of metal-poor and old stars seems to have larger velocities in the direction of the Galactic centre $\left(\left|U_{\mathrm{LSR}}\right|>40 \mathrm{~km} \mathrm{~s}^{-1}\right)$ and larger eccentricities $(e \gtrsim 0.2)$ than the other stars. The star HD 50806 appears to have a singular position in this figure (in particular, it has the most eccentric orbit among the sample stars), which is probably related to its membership in the transition population of thin-thick disc stars. The limitation of our sample does not allow to verify the results of Rocha-Pinto et al. (2006) that metal-poor and old stars show more orbital radial spread in the Galaxy.

\subsection{Abundance trends as a function of $[\mathrm{Fe} / \mathrm{H}]$}

Through the analysis of Fig. 8 we investigate possible trends in the abundance ratios as a function of the stellar metallicity. These trends are more clearly identified if the elements are grouped together, either based on their nucleosynthetic origin or because they share similar trends in the diagrams. For this reason, we show in the bottom panels of this figure the mean abundance $\langle[\mathrm{X} / \mathrm{Fe}]\rangle$ of a few groups of elements as a function of the metallicity (the same groups plotted in Fig. 7 and listed in Table 7). The stars are also identified according to the tree clustering analysis. We have fitted linear regressions on the diagrams in three ranges of metallicity: for stars poorer than the Sun, for stars of solar metallicity or richer, and for all the sample stars. We have then computed the cross-correlation coefficients in these three metallicity ranges and plotted the regressions of the more significant trends (only if $|r| \geq 0.5$ ).

The overall trend of our abundance ratios as a function of the stellar metallicity normally follows what has been suggested in the literature concerning the nucleosynthetic origin of the elements and their abundance evolution in time (Chen et al. 2000; Reddy et al. 2003; Bensby et al. 2005; Chen et al. 2008; Neves et al. 2009). The light metals $\mathrm{Ca}, \mathrm{Sc}$, and $\mathrm{Ti}$ are predominantly produced by type II Supernovae (SN II) at the beginning of the enrichment history of the Galactic disc. On the other hand, iron and the iron-peak elements $\mathrm{V}, \mathrm{Cr}, \mathrm{Co}$, and $\mathrm{Ni}$ are predominantly synthesised by type Ia Supernovae (SN Ia) in longer time scales. Therefore, it is expected that the abundance ratio of these light metals with respect to iron progressively decrease from metal-poor to metal-rich stars, whereas the abundance ratio of iron-peak elements with respect to iron, all produced at the same rate, remains constant and close to zero in the whole range of metallicity. Indeed, this is exactly what is observed in Fig. 8 for $[\mathrm{Ca}, \mathrm{Sc}, \mathrm{Ti} / \mathrm{Fe}]$ and $[\mathrm{V}, \mathrm{Cr}, \mathrm{Co}, \mathrm{Ni} / \mathrm{Fe}]$, to within our stated abundance uncertainties.

The light metals $\mathrm{Mg}$ and $\mathrm{Si}$ may not only be produced by SN II considering that $[\mathrm{Mg}, \mathrm{Si} / \mathrm{Fe}]$ flattens out for metallicities higher than -0.1 dex. The same behaviour was found by Chen et al. (2000) and Neves et al. (2009), who suggested that SN Ia is possibly contributing. The star HD 50806 is clearly enriched in $\mathrm{Mg}$ (and in other elements as well) according to Fig. 8, probably reflecting its membership to the thin-thick disc transition.

The situation of $\mathrm{Mn}, \mathrm{Cu}$, and $\mathrm{Zn}$ is somewhat more complex. The hypothesis of production in SN Ia still stands, but this is probably not the unique source. Allen \& Porto de Mello (2011), in their study of s-process enriched stars, suggested that SN Ia is the main source of production of manganese, in opposition to the conclusions of Feltzing et al. (2007), who suggested that this element is mainly produced by SN II. Our results in Fig. 8, which show an increasing trend of $[\mathrm{Mn} / \mathrm{Fe}]$ as a function of $[\mathrm{Fe} / \mathrm{H}]$ (see also the bottom panel of Fig. 6, in which there is a sequential crescent ordination of $\langle[\mathrm{X} / \mathrm{Fe}]\rangle$ from the metal-poor clustering group to the metal-rich one), seem to support the idea of an extra nucleosynthetic source for the Mn yields. Such an increasing trend is also usually attributed to a metallicity dependence in the production of Mn in both SN Ia and SN II. In this work, $\mathrm{Mn}$ and $\mathrm{Cu}$ were plotted together through the mean abundance ratio $[\mathrm{Mn}, \mathrm{Cu} / \mathrm{Fe}]$. Both these elements have abundances that increase with metallicity, though for $\mathrm{Cu}$ this trend is not as significant as for $\mathrm{Mn}$, and seems to happen only for higher metallicities, being constant and close to zero for $[\mathrm{Fe} / \mathrm{H}]<0$. $\mathrm{Cu}$ and $\mathrm{Zn}$, although being adjacent elements in the periodic table, stand in the transition between iron-peak and s-process elements, and their behaviour is in sharp contrast. A decreasing trend in $[\mathrm{Zn} / \mathrm{Fe}]$ vs. $[\mathrm{Fe} / \mathrm{H}]$ is seen for stars poorer than the Sun, in agreement with Fig. 1 of Allen \& Porto de Mello (2011).

The elements $\mathrm{C}$ and $\mathrm{Na}$ were not grouped together with other elements. $\mathrm{C}$ is synthesised in several different sites and behaves similarly to $\mathrm{O}$ and $\mathrm{N}$, with $[\mathrm{C} / \mathrm{Fe}]$ decreasing with increasing metallicity. This negative trend is mostly observed in the metalpoor regime $([\mathrm{Fe} / \mathrm{H}]<-0.3)$, hence not seen in Fig. 8 given the limited metallicity range of our programme stars. Nevertheless, our results agree very well with the recent $\mathrm{C}$ abundance determination performed by da Silva et al. (2011) for solar-like dwarfs. $\mathrm{Na}$ is probably produced, among other processes, in the core of massive stars and ejected by SN II into the interstellar medium. Here we found that $[\mathrm{Na} / \mathrm{Fe}]$ is constant and nearly close to zero in the range of metal-poor stars, with a possible increasing trend for higher metallicities. Chen et al. (2000) suggested that maybe $[\mathrm{Na} / \mathrm{Fe}]$ is close to zero for the whole metallicity range of disc stars. Neves et al. (2009), however, found that $[\mathrm{Na} / \mathrm{Fe}]$ is close to zero for thin disc stars for $[\mathrm{Fe} / \mathrm{H}]$ between -0.2 and +0.2 , but above solar for other values of metallicity.

The $[\mathrm{X} / \mathrm{Fe}]$ abundance ratios of elements of the s-process, mainly produced in TP-AGB of intermediate or low mass stars, and the r-process, produced in sites with high neutron density such as the final stages of massive stars (SN II, neutron stars), are supposed, respectively, to progressively increase and decrease from metal-poor stars to higher metallicities. These facts reflect the production of these elements in different time scales with respect to iron, the former products of long-lived AGB stars, the latter arising from short-lived massive stars which explode as supernovae. This is not clearly observed in the diagrams of Fig. 8 for $\mathrm{Sm}$ and the s-process elements because of the short metallicity range. An interesting result of the analysis of these diagrams are the properties involving some Ba-enriched stars, which we discuss in the next section.

\subsection{Abundance trends as a function of $[\mathrm{Ba} / \mathrm{Fe}]$}

A few stars in our sample are remarkably enriched in Ba by more than $3 \sigma$, especially HD 39587 and HD 147513. For this reason we also investigated the behaviour of the abundance ratios $[\mathrm{X} / \mathrm{Fe}]$ or $\langle[\mathrm{X} / \mathrm{Fe}]\rangle$ as a function of $[\mathrm{Ba} / \mathrm{Fe}]$ for some elements or groups of elements showing some kind of relation with the production of barium (see Fig. 9). Once more, we computed the cross-correlations coefficients and we plotted in the figure the regressions of the most significant trends $(|r| \geq 0.5)$. 
A\&A 542, A84 (2012)
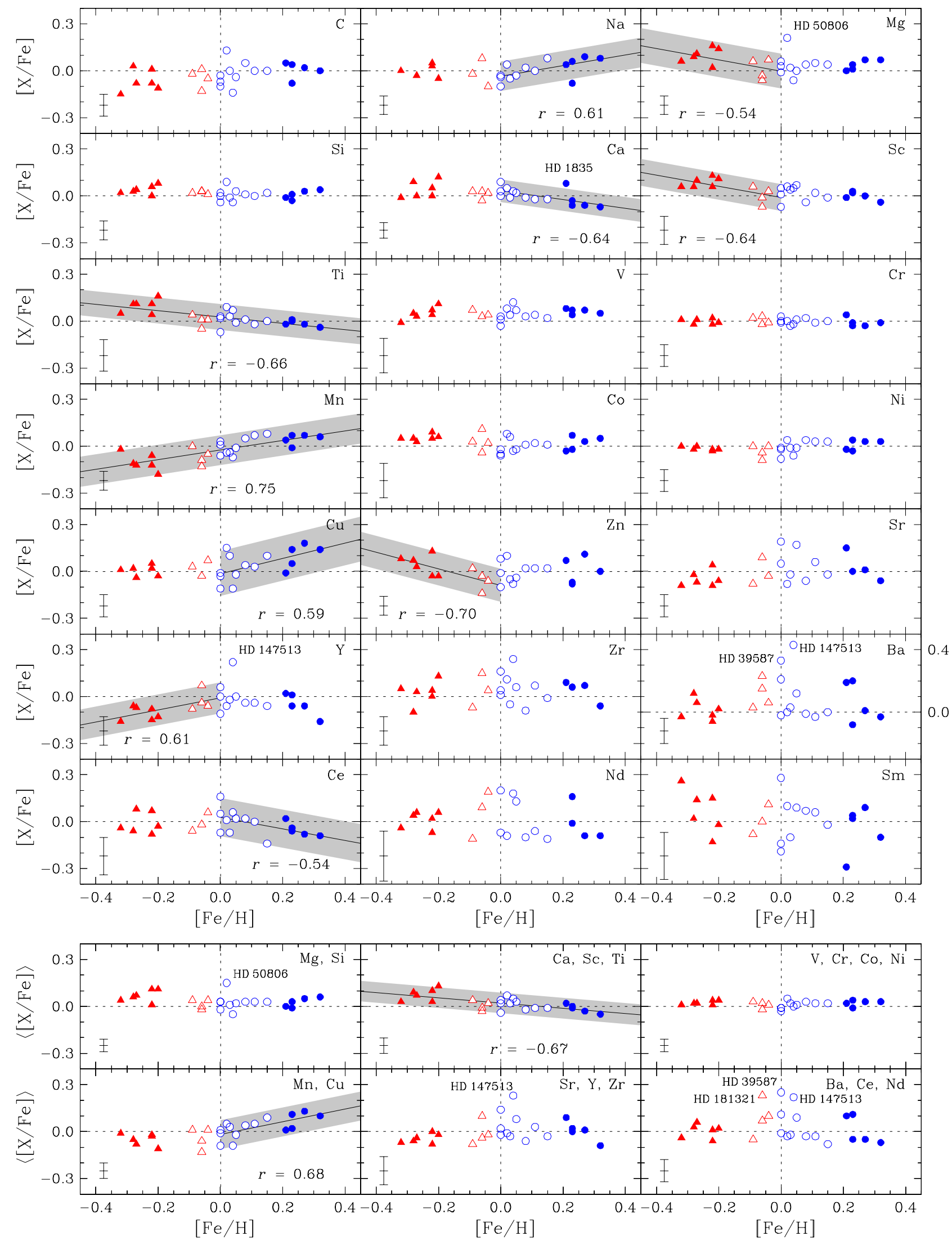

Fig. 8. Abundance ratios as a function of the stellar metallicity for individual elements (top panels) and for nucleosynthetic groups (bottom panels). The ordinate axis has a different scale for $[\mathrm{Ba} / \mathrm{Fe}]$ due to its larger abundances. The linear regressions (solid line), the $95 \%$ confidence intervals (hashed area), and the cross-correlation coefficients are also shown for $|r| \geq 0.5$. The symbols follow the classification of Sect. 5 (see Fig. 6). 


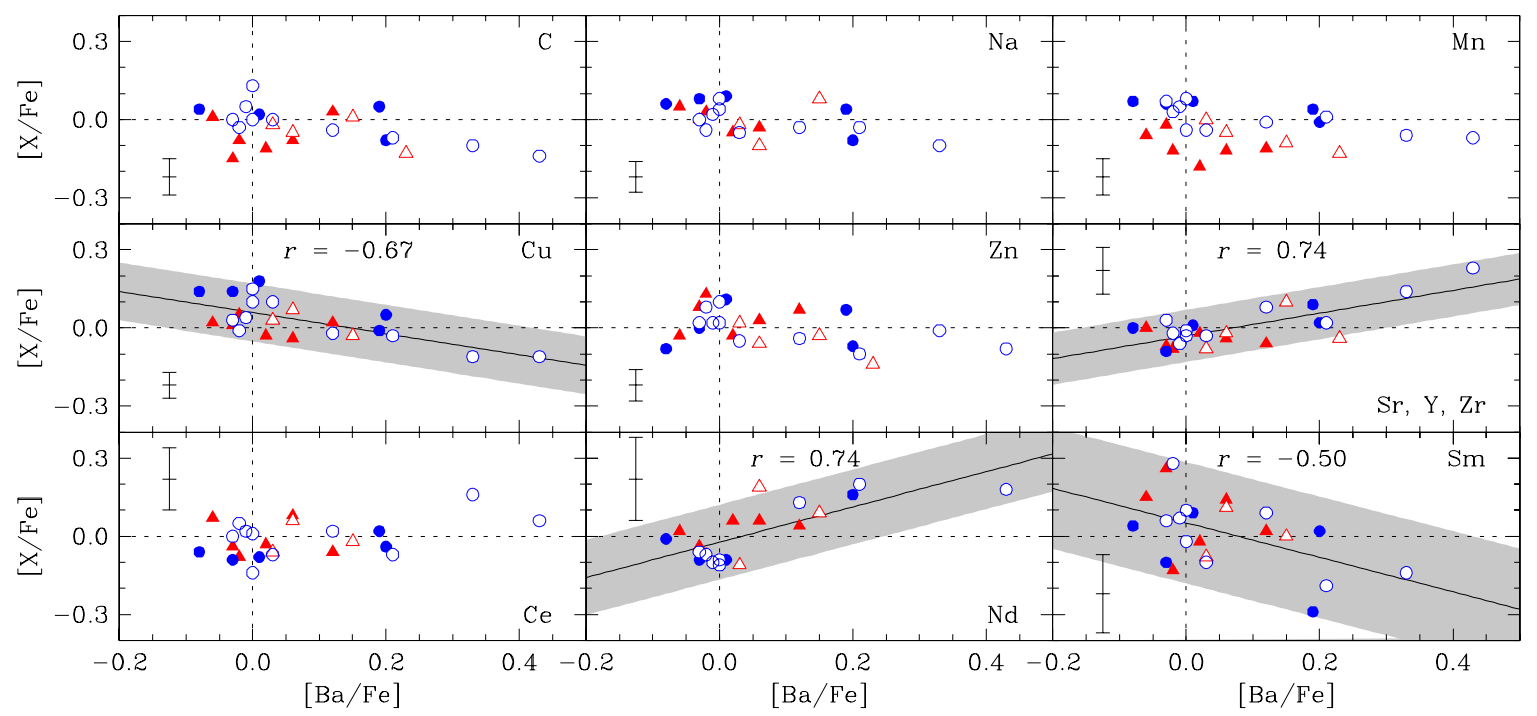

Fig. 9. Abundance ratios as a function of $[\mathrm{Ba} / \mathrm{Fe}]$. For the elements of the light s-process $(\mathrm{Sr}, \mathrm{Y}$, and $\mathrm{Zr})$ the mean abundance ratios are plotted. The linear regressions (solid line), the $95 \%$ confidence intervals (hashed area), and the cross-correlation coefficients are also shown for $|r| \geq 0.5$. The symbols follow the classification of Sect. 5 (see Fig. 6).

Castro et al. (1999) proposed the existence of an anticorrelation between the abundances of $\mathrm{Cu}$ and the s-process elements. They found that $[\mathrm{Cu} / \mathrm{Fe}]$ decreases with the increasing of $[\mathrm{Ba} / \mathrm{Fe}]$, suggesting a relation between the destruction of $\mathrm{Cu}$ and the production of $\mathrm{Ba}$ (and other s-process elements). In the recent analysis of Ba-enriched stars of Allen \& Porto de Mello (2011), the authors have not supported this scenario, arguing that $\mathrm{Cu}$ seems to be little (or not at all) affected by the s-process, even though they have acknowledged that some Ba-rich stars do present anticorrelated abundances of $\mathrm{Cu}$ and the s-process elements. Our results point to a statistically significant decrease in the abundances of $\mathrm{Cu}$ with increasing $[\mathrm{Ba} / \mathrm{Fe}]$, in accordance with Castro et al. (1999).

Two other iron-peak elements, $\mathrm{Mn}$ and $\mathrm{Zn}$, are also shown in Fig. 9 and no clear correlation is observed. This may indicate that $\mathrm{Mn}$ and $\mathrm{Cu}$ do not share the same nucleosynthetic origin. Indeed, Allen \& Porto de Mello (2011) found that the synthesis of $\mathrm{Cu}$ receives a larger contribution from not so massive stars than $\mathrm{Zn}$, a result roughly in line with those of Castro et al. (1999) and ours. Such results point towards the necessity of both more extensive observations of the abundances of $\mathrm{Cu}$ and $\mathrm{Zn}$, and more protracted theoretical efforts, in order that a better understanding of the complex chemical history of these two elements may be achieved.

Castro et al. (1999) also proposed an anticorrelation in the abundances of $\mathrm{C}$ and $\mathrm{Na}$ with respect to $[\mathrm{Ba} / \mathrm{Fe}]$. Our results do not seem to support this anticorrelation, though our most Ba-rich stars, the Ursa Major group members HD 39587 and HD 147513, are markedly C-deficient. Porto de Mello \& da Silva (1997a) attributed the $[\mathrm{C} / \mathrm{Fe}]$ deficiency of a barium star to the ${ }^{13} \mathrm{C}(\alpha, n){ }^{16} \mathrm{O}$ reaction that occurred in the hot-bottom envelope of its companion during the TP-AGB phase. However, HD 39587 and HD 147513 are no longer regarded as true barium stars.

Figure 9 shows an evident expected correlation for the light s-process elements ( $\mathrm{Sr}, \mathrm{Y}$, and $\mathrm{Zr}$ ). Correlations involving $\mathrm{Nd}$, another heavy element of the s-process may also exist, but its abundance determination has larger uncertainties. An anticorrelation between $[\mathrm{Sm} / \mathrm{Fe}]$ and $[\mathrm{Ba} / \mathrm{Fe}]$ also seems to exist. Sm is a good representative of the r-process elements, and despite the very large uncertainties in the determination of such elements, usually showing very few lines in the spectra of solar-type stars, an interpretation in which this anticorrelation is due to ever more efficient production of s-process elements in AGB stars, as compared to the production of the r-process in SN II, seems warranted.

\subsection{Abundance trends as a function of age}

One of the motivations of the present paper is to explore the abundance ratios of elements due to different nucleosynthetic processes and the stellar ages, taking advantage of the reasonably precise ages that can be attributed to our programme stars. Figure 11 shows the diagrams $[\mathrm{X} / \mathrm{Fe}]$ and $\langle[\mathrm{X} / \mathrm{Fe}]\rangle$ as a function of the stellar age, and Fig. 12 explores the relation between the abundance ratios $\left[\mathrm{X}_{1} / \mathrm{X}_{2}\right]$ of different elements and age. Similarly to Fig. 8, we have fitted linear regressions on the diagrams in three ranges of stellar age: for stars younger than the Sun (age $<4.53 \mathrm{Gyr}$ ), for stars older than the Sun, and for all the sample stars. We have then computed the cross-correlation coefficients in these three ranges of stellar age and plotted the regressions of the more significant trends (only if $|r| \geq 0.5$ ). In these figures there are positive, negative, or flat abundance trends in the three ranges of age. We notice, however, that the age of the Sun, used as a reference, was arbitrarily chosen. The exact value of the transition age when the abundance behaviour changes is not clear from these plots (it is a value between 4 and $6 \mathrm{Gyr}$ ).

In spite of the long recognition (though not undisputed) of the so-called age-metallicity relation (see Fig. 10), the individual abundances in Figs. 11 and those in 8 do not share exactly the same behaviour, leading us to suggest in the following that the age-metallicity relation may be a multidimensional concept. For this reason, we regrouped the elements according to their abundance behaviour with age (see the bottom panels of Fig. 11).

Carbon and sodium do not seem to present any important trends of $[\mathrm{X} / \mathrm{Fe}]$ with age. The positive trends observed for $\mathrm{Mg}$, $\mathrm{Sc}$, and $\mathrm{Ti}$ (less clearly seen for Si and Ca) are simply the result of the Galactic chemical evolution. The production rate of these elements by SN II decrease with time since the formation of the Galactic disc (equivalently to increasing with age) compared to the increased production of Fe by the longer-lived SN Ia as we 


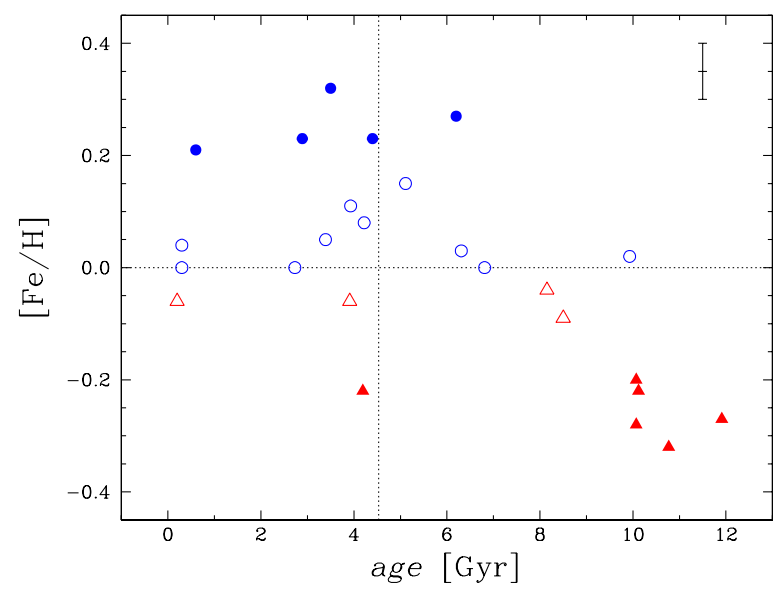

Fig. 10. Stellar metallicity as a function of age. The symbols follow the classification of Sect. 5 (see Fig. 6).

approach more recent epochs. Silicon perhaps shows no trend at all; $\mathrm{Mg}$ seems to have a more or less positive linear trend with increasing age; in their turn $\mathrm{Ca}, \mathrm{Sc}$, and Ti sport a more complex behaviour. The statistical significance of the behaviour of $\mathrm{Ca}$ is slight, and not much confidence should be placed in the apparent $[\mathrm{Ca} / \mathrm{Fe}]$ decrease with time, followed by an increase towards more recent times. Taken at face value, this would appear to lend support to the suggestion that some fraction of the Ca synthesis might be due to SN Ia, in unison with their production of Fe. Sc and Ti seem to have a significant decrease with time with respect to Fe, but this decrease stops at a time close to the solar age and flattens thereafter towards present times.

Among the Fe-peak elements, no important trend is seen in the $[\mathrm{X} / \mathrm{Fe}]$ relation with age for $\mathrm{V}, \mathrm{Cr}, \mathrm{Co}$, and $\mathrm{Zn}$; only a dubious one for $\mathrm{Ni}$ in the interval of young stars. Yet, again, $\mathrm{Cu}$ and $\mathrm{Mn}$ suggest more underlying complexity. Even though the statistical significance of the linear regressions is slight, the abundance ratios to $\mathrm{Fe}$ of both these elements seem first to increase towards the present epoch, and then decrease (a behaviour that is reinforced when these two elements are plotted together through the mean abundance ratio $[\mathrm{Mn}, \mathrm{Cu} / \mathrm{Fe}]$ ). Recalling that Allen \& Porto de Mello (2011) have found that $\mathrm{Mn}$ is mostly due to SN Ia, our result could imply that the relative yield of Mn to Fe in SN Ia decreases with time (and consequently the overall metallicity). The situation for $\mathrm{Cu}$ is less straightforward, as usual. Allen \& Porto de Mello (2011) suggest that little of the synthesis of $\mathrm{Mn}, \mathrm{Cu}$, and $\mathrm{Zn}$ is owed to the main s-process, leaving the action of AGB stars an unlikely source of such a behaviour. These same authors assert that the action of the so-called weak s-process, sited at the He-burning core of massive stars, has a non negligible contribution to the synthesis of $\mathrm{Mn}, \mathrm{Cu}$, and $\mathrm{Zn}$. One possible explanation for the decrease of the abundance ratios of $[\mathrm{Mn} / \mathrm{Fe}]$ and $[\mathrm{Cu} / \mathrm{Fe}]$ towards more recent times is a decreasing yield of the weak s-process in their synthesis, as contrasted to the production of Fe by SN Ia. Clearly, an understanding of the detailed chemical evolution of elements, in both the dimensions of metallicity and age, of the Fe-peak and its transition with the heavier elements deserves closer scrutiny, both observationally and theoretically.

We next turn to the $[\mathrm{X} / \mathrm{Fe}]$-age relation for the s-process elements. These present particular interest, since Castro et al. (1999) suggested, also using data from Edvardsson et al. (1993), that $[\mathrm{Ba} / \mathrm{H}]$ has a steeper decrease with age than $[\mathrm{Fe} / \mathrm{H}]$, and therefore that $[\mathrm{Ba} / \mathrm{Fe}]$ increases towards modern times (see also
Bensby et al. 2007). This fact might be interpreted, again, as a consequence of the larger yield of the s-process element synthesis by the long-lived AGB stars in relation to the not aslong-lived production of Fe by SN Ia. Do the other s-process elements show a behaviour similar to barium? Apparently this is so, but not in a straightforward way. The lighter s-process elements $\mathrm{Sr}$ and $\mathrm{Y}$ seem to have a linear trend of $[\mathrm{X} / \mathrm{Fe}]$ with age (clearly seen for $\mathrm{Sr}$ ), increasing towards present times, as expected. The $[\mathrm{Zr} / \mathrm{Fe}],[\mathrm{Ce} / \mathrm{Fe}]$, and $[\mathrm{Nd} / \mathrm{Fe}]$ ratio, however, appears flat in the old age regime, possibly increasing only for stars younger than the Sun. The $[\mathrm{Ba} / \mathrm{Fe}]$ ratio behaves similarly but the increase towards younger ages is much sharper and more significant. At face value, these results point towards the evolution of the relative yields of such s-process elements with time (and metallicity) in AGB stars, apparently favouring the heavier species $\mathrm{Ba}$ and $\mathrm{Nd}$ over lighter ones. The simultaneous analysis of the $[\mathrm{Cu} / \mathrm{Fe}]$ and [s-process/Fe] ratios, epitomised, for example, by the $[\mathrm{Ba} / \mathrm{Fe}]$ relation with age, suggests an anticorrelation of $\mathrm{Cu}$ and $\mathrm{Ba}$ towards younger stars, as found by Castro et al. (1999), adding to the controversy surrounding the chemical evolution of copper.

Finally, a positive trend with increasing age is observed for $[\mathrm{Sm} / \mathrm{Fe}]$ in stars younger than the Sun, again reflecting the smaller number of SN II than SN Ia in the present in comparison with the past (in this case the epoch of the Sun's formation). Significant positive trends are also observed for $\mathrm{Ni}$ and $\mathrm{Cu}$ in the regime of younger stars.

Concerning the four groups yielded by the tree clustering analysis and their relation with age, we can only state that, as expected, the old stars in our sample are also metal-poor, whereas young stars tend to be metal-rich. Once more, the case of the star HD 50806 is evidenced. Although classified in the intermediate group of stars with slightly over-solar abundances $(\langle[\mathrm{X} / \mathrm{H}]\rangle=+0.06 \mathrm{dex})$, it is situated close to the group of metalpoor stars $(\langle[\mathrm{X} / \mathrm{H}]\rangle=-0.24 \mathrm{dex})$ in Fig. 11, which is maybe a consequence of its population membership.

The bottom panels of Fig. 11 shows the average of the $[\mathrm{X} / \mathrm{Fe}]$ relations with age for selected groups of elements. Thus we see that grouping $\mathrm{Sc}$ and $\mathrm{Ti}$ reinforces the relation with age already shown by each element individually, and the same is seen for the grouping of $\mathrm{Sr}$ and Y. Similarly, averaging the $[\mathrm{X} / \mathrm{Fe}]$ relations with age for $\mathrm{Zr}, \mathrm{Ba}$, and $\mathrm{Nd}$ produces a very steep increase towards present epochs, after a flat relation from the birth of the Galactic disc up to the solar age.

These results prompted us to investigate the specifics of the $\left[\mathrm{X}_{1} / \mathrm{X}_{2}\right]$ ratios with age, where $\mathrm{X}_{1}$ and $\mathrm{X}_{2}$ designate elements other than Fe. In Fig. 12 we explore the time evolution of some elements that displayed a particular clear $[\mathrm{X} / \mathrm{Fe}]$ relation with age. Thus, it is apparent that the $[\mathrm{Ba} / \mathrm{Mg}]$ and $[\mathrm{Ba} / \mathrm{Zn}]$ increase steeply towards present epochs, for stars younger than the Sun, reinforcing their individual and opposite behaviour in the $[\mathrm{X} / \mathrm{Fe}]$-age diagrams. Also, both $[\mathrm{Sr} / \mathrm{Mg}]$ and $[\mathrm{Y} / \mathrm{Mg}]$ increase linearly and significantly from the oldest to the youngest stars; the same is seen in the $[\mathrm{Sr} / \mathrm{Zn}]$ and $[\mathrm{Y} / \mathrm{Zn}]$ ratios. Investigating the $\left[\mathrm{X}_{1} / \mathrm{X}_{2}\right]$ ratios of s-process to $\mathrm{r}$-process elements, we found steep increases in the $[\mathrm{Ba} / \mathrm{Sm}],[\mathrm{Sr} / \mathrm{Sm}]$, and $[\mathrm{Y} / \mathrm{Sm}]$ ratios towards younger stars, but only for objects younger than the Sun. Significant age relations are, therefore, evidenced in the $\left[\mathrm{X}_{1} / \mathrm{X}_{2}\right]$ ratios of diverse elements, representing a wide range of nucleosynthetic processes and tentatively allowing the proposition that the age-metallicity relation is a more complex constraint to Galactic chemo-dynamical models than hitherto recognised. 
R. da Silva et al.: Chemo-chronological analysis of solar-type stars
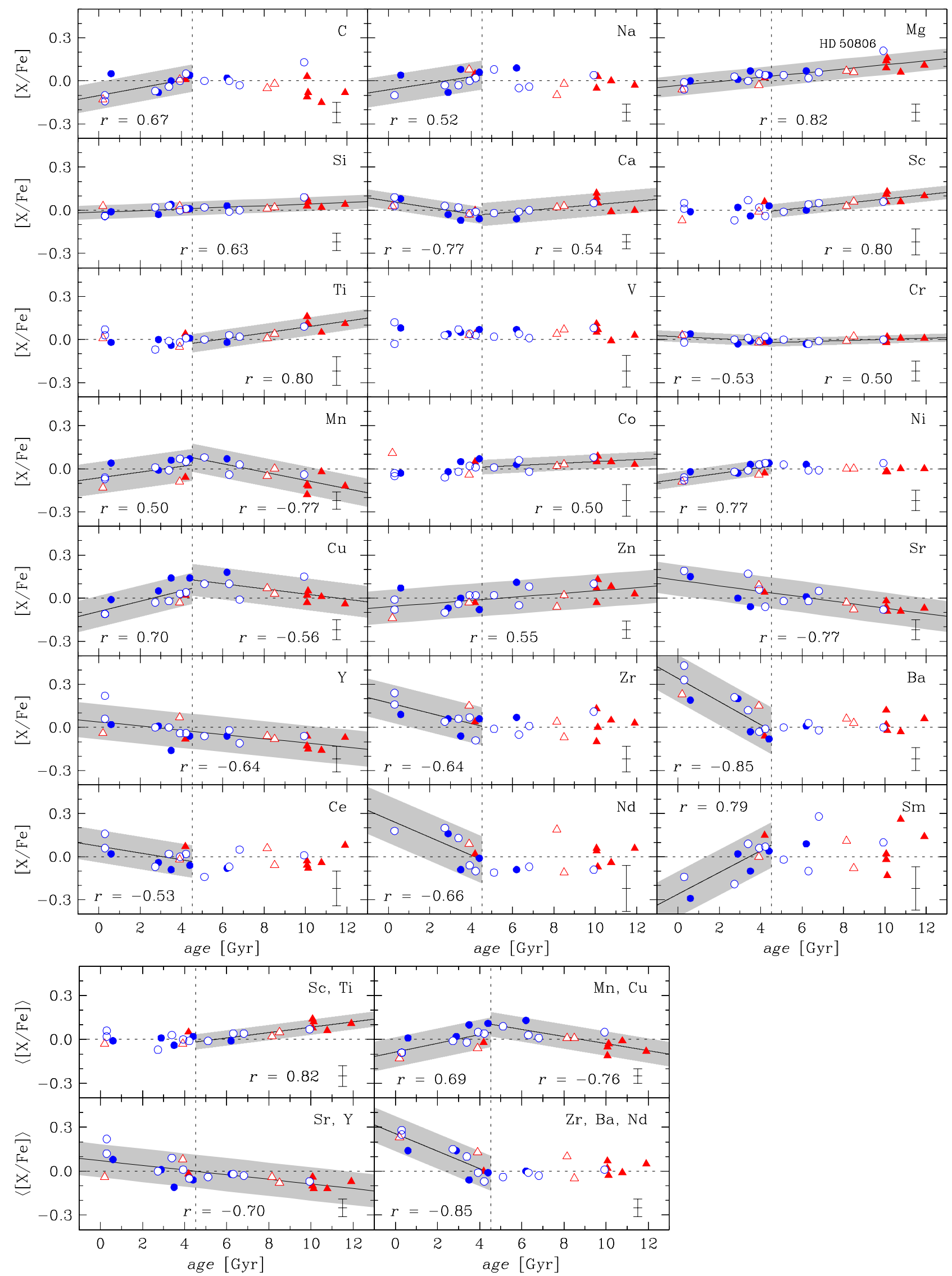

Fig. 11. Abundance ratios as a function of the stellar age for individual elements (top panels) and for nucleosynthetic groups (bottom panels). The vertical dashed line indicates the adopted solar age ( $4.53 \mathrm{Gyr}$ ). The linear regressions (solid line), the 95\% confidence intervals (hashed area), and the cross-correlation coefficients are also shown for $|r| \geq 0.5$. The symbols follow the classification of Sect. 5 (see Fig. 6). 


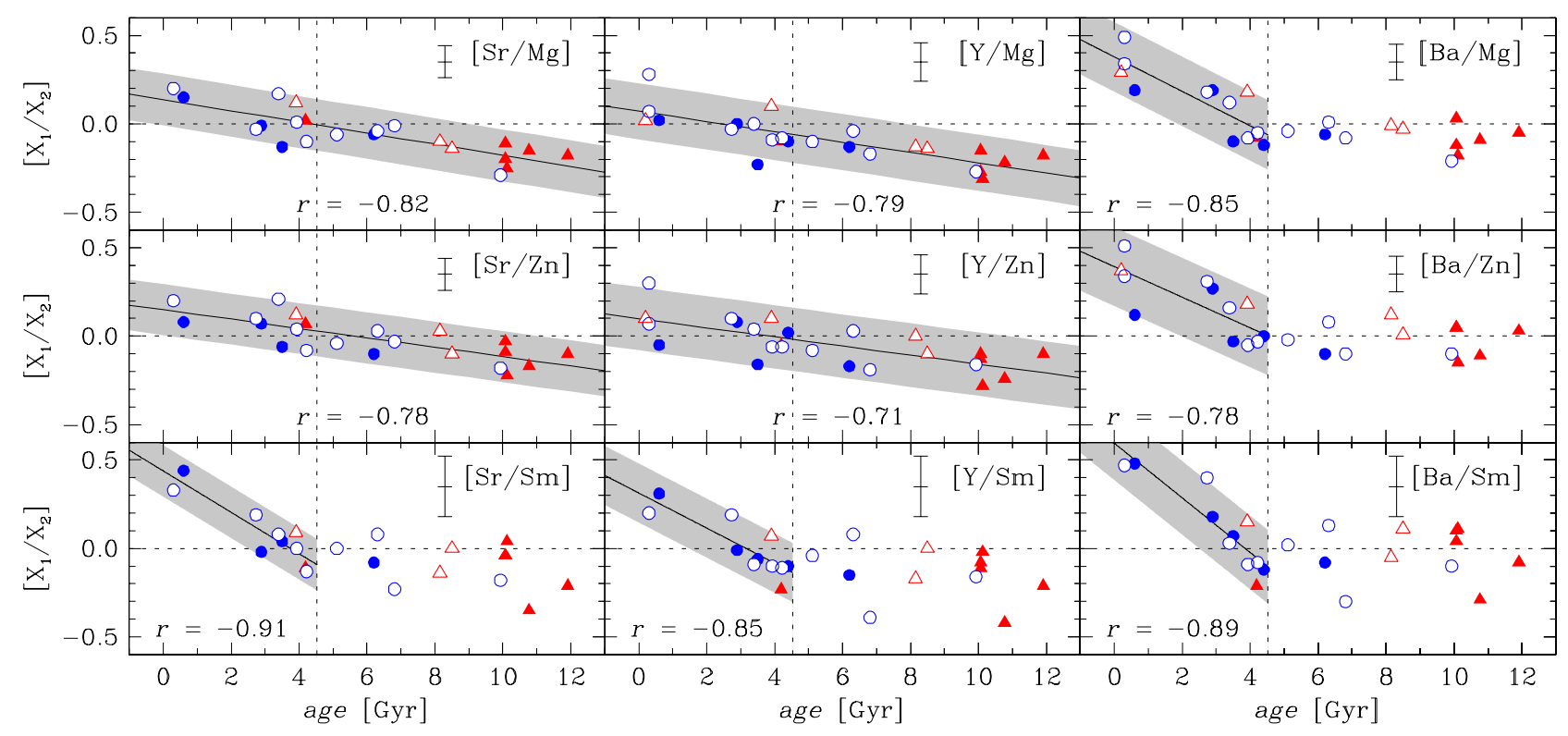

Fig. 12. Abundance ratios as a function of the stellar age. The vertical dashed line indicates the adopted solar age (4.53 Gyr). The linear regressions (solid line), the $95 \%$ confidence intervals (hashed area), and the cross-correlation coefficients are also shown for $|r| \geq 0.5$. The symbols follow the classification of Sect. 5 (see Fig. 6).

\subsection{Abundance trends with condensation temperature}

Our determination of multi-elemental abundances also provides the study of possible trends in the abundance ratios $[\mathrm{X} / \mathrm{Fe}]$ as a function of the condensation temperature $\left(T_{\mathrm{C}}\right)$ of each element. Values of $50 \% T_{\mathrm{C}}$ (the temperature when $50 \%$ of an element is in the condensed phase) for a solar-system composition gas were taken from Lodders (2003) and Lodders et al. (2009).

For a few stars in our sample we have found some correlations of $[\mathrm{X} / \mathrm{Fe}]$ with $T_{\mathrm{C}}$ (even after corrections due to Galactic chemical evolution effects were applied). However, no clear correlation seems to exist when comparing the slopes for refractory elements (those with $T_{\mathrm{C}} \gtrsim 900 \mathrm{~K}$ ) with several stellar parameters $\left(T_{\text {eff }}^{\text {mean }},[\mathrm{Fe} / \mathrm{H}], \log g, \xi\right.$, mass, and age $)$. The one involving the metallicity was proposed by Ramirez et al. (2010) in the sense that higher-metallicity stars present more negative slopes. Our results agree with their paper, but the number of metal-rich stars in our sample is too small to confirm their conclusions.

\section{Conclusions}

In this work we have performed a multi-elemental, differentially with respect to the Sun, spectroscopic analysis of a sample of 25 solar-type stars in the solar neighbourhood. We have derived their atmospheric parameters (from various nearly independent criteria and with low internal errors), masses, ages, kinematical and orbital parameters, and elemental abundances (derived with very low internal uncertainties) based on equivalent widths or spectral synthesis.

Despite small in size, our sample was carefully selected to undergo an homogeneous and detailed analysis based on spectra with high resolution and high $\mathrm{S} / \mathrm{N}$. We have: (i) checked the effective temperatures based on the excitation equilibrium of neutral iron lines against those from photometric calibrations and from the $\mathrm{H} \alpha$ wings profile; (ii) checked the surface gravities computed through the ionisation equilibrium between $\mathrm{Fe}_{\mathrm{I}}$ and Fe II lines against those computed based on the evolutionary parameters; (iii) derived masses and ages from evolutionary tracks and isochrones computed considering the metallicity of each star; and (iv) applied a differential spectroscopic analysis relative to the Sun, hence minimising the systematic errors and yielding a mean uncertainty of $0.06 \mathrm{dex}$ in the abundance ratios. We thus expect that our determinations have achieved a high level of precision and accuracy.

We have also applied a statistical study to our abundance results using the method of tree clustering analysis, through which we looked for groupings of stars that share similar abundances in the $[\mathrm{X} / \mathrm{H}]$ space. Although our sample has a limited range in metallicity, it covers a broad range in age. The detailed abundance pattern was then investigated through correlations with kinematics, Galactic orbits, and stellar ages. Our conclusions are thus summarised:

1- Four groups were identified, two having over-solar abundances (with averages +0.26 and +0.06 dex on $[\mathrm{X} / \mathrm{H}]$ ), and two with under-solar abundance values (on average -0.06 and -0.24 dex). Possible non-solar abundance ratios, even for stars which share the same age, Galactic orbit, and metallicity as the Sun, are suggested. Whether these are due to heterogeneity in the stellar natal clouds, or by dynamical migration within the Galactic disc, should be investigated with larger samples. In particular, the results of Rocha-Pinto et al. (2006) that metal-poor and old stars show more orbital radial spread in the Galaxy could not be verified given the limitation of our sample.

2- The presence of Ba-enriched stars in our sample prompted us to investigate in detail the relation of some elements with $\mathrm{Ba}$. An anticorrelation between $[\mathrm{Cu} / \mathrm{Fe}]$ and $[\mathrm{Ba} / \mathrm{Fe}]$ was found, in line with similar claims in the literature. The $[\mathrm{Sm} / \mathrm{Fe}]$ abundance ratios seem to be anticorrelated with $[\mathrm{Ba} / \mathrm{Fe}]$, barely at the $95 \%$ confidence level. On the other hand, previous suggestions of $[\mathrm{C} / \mathrm{Fe}]$ and $[\mathrm{Na} / \mathrm{Fe}]$ anticorrelations with $[\mathrm{Ba} / \mathrm{Fe}]$ could not be confirmed. Even though the possible connected chemical evolution of $\mathrm{Mn}$, $\mathrm{Cu}$, and $\mathrm{Zn}$ has been recently discussed in the literature, no significant trend of $[\mathrm{Mn} / \mathrm{Fe}]$ and $[\mathrm{Zn} / \mathrm{Fe}]$ with $[\mathrm{Ba} / \mathrm{Fe}]$ is suggested. 
R. da Silva et al.: Chemo-chronological analysis of solar-type stars

Table 8. Atomic line parameters of the elements used in the analysis.

\begin{tabular}{|c|c|c|c|c|c|c|c|c|c|c|c|c|c|}
\hline \multirow[t]{2}{*}{$\lambda[\AA]$} & \multirow[t]{2}{*}{ Id. } & \multirow{2}{*}{$\begin{array}{c}\chi \\
{[\mathrm{eV}]}\end{array}$} & \multicolumn{2}{|c|}{ Gany 1} & \multicolumn{2}{|c|}{ Gany 2} & \multirow[t]{2}{*}{$\lambda[\AA ̊]$} & \multirow[t]{2}{*}{ Id. } & \multirow{2}{*}{$\begin{array}{c}\chi \\
{[\mathrm{eV}]}\end{array}$} & \multicolumn{2}{|c|}{ Gany 1} & Gan & \\
\hline & & & $\log g f$ & $E W$ & $\log g f$ & $E W$ & & & & $\log g f$ & $E W$ & $\log g f$ & $E W$ \\
\hline 5052.167 & $\mathrm{C}_{\mathrm{I}}$ & 7.68 & -1.48 & - & -1.62 & - & 5147.479 & Ti I & 0.00 & - & - & -1.98 & 42.4 \\
\hline 5380.322 & $\mathrm{C}_{\mathrm{I}}$ & 7.68 & -1.78 & - & -1.84 & - & 5152.185 & Ti I & 0.02 & - & - & -2.03 & 39.1 \\
\hline 6154.230 & $\mathrm{Na}$ & 2.10 & -1.52 & 42.3 & -1.52 & 40.8 & 5192.969 & Ti I & 0.02 & -1.10 & 88.3 & -1.02 & 84.4 \\
\hline 6160.753 & $\mathrm{Na}$ & 2.10 & -1.23 & 61.8 & -1.29 & 59.9 & 5211.206 & Ti I & 0.84 & -2.10 & 9.1 & -2.07 & 9.2 \\
\hline 4571.102 & $M g_{I}$ & 0.00 & - & 107.8 & - & 110.3 & 5219.700 & Ti I & 0.02 & -2.32 & 27.9 & -2.23 & 30.0 \\
\hline 4730.038 & $M g_{I}$ & 4.34 & - & 78.2 & - & 70.9 & 5295.780 & Ti I & 1.07 & - & - & -1.60 & 13.2 \\
\hline 5711.095 & $\mathrm{Mg}_{\text {I }}$ & 4.34 & - & 119.2 & - & 106.4 & 5426.236 & Ti I & 0.02 & - & - & -2.97 & 7.8 \\
\hline 5785.285 & $\mathrm{Mg}_{\mathrm{I}}$ & 5.11 & -1.87 & 50.5 & -1.82 & 55.3 & 5471.197 & Ti I & 1.44 & - & - & -1.48 & 9.6 \\
\hline 5517.533 & $\mathrm{Si}$ I & 5.08 & -2.42 & 15.7 & -2.51 & 13.0 & 5490.150 & $\mathrm{Ti}_{\mathrm{I}}$ & 1.46 & - & - & -1.00 & 22.6 \\
\hline 5621.607 & $\mathrm{Si}_{\mathrm{I}}$ & 5.08 & - & _- & -2.61 & 10.5 & 5648.567 & Ti I & 2.49 & -0.40 & 11.5 & -0.39 & 11.1 \\
\hline 5665.563 & $\mathrm{Si}_{\mathrm{I}}$ & 4.92 & -1.98 & 42.2 & -1.96 & 41.8 & 5679.937 & Ti I & 2.47 & -0.65 & 6.9 & -0.63 & 7.0 \\
\hline 5684.484 & $\mathrm{Si} \mathrm{I}_{\mathrm{I}}$ & 4.95 & - & - & -1.60 & 62.3 & 5739.464 & $\mathrm{Ti}_{\mathrm{I}}$ & 2.25 & -0.67 & 10.5 & -0.75 & 8.6 \\
\hline 5690.433 & Si I & 4.93 & -1.80 & 52.9 & -1.81 & 50.6 & 5866.452 & $\mathrm{Ti}_{\mathrm{I}}$ & 1.07 & - & - & -0.82 & 49.6 \\
\hline 5701.108 & $\mathrm{Si}_{\mathrm{I}}$ & 4.93 & -1.90 & 46.3 & -1.97 & 41.0 & 6064.629 & Ti I & 1.05 & - & - & -1.88 & 9.7 \\
\hline 5708.405 & $\mathrm{Si}_{\mathrm{I}}$ & 4.95 & - & - & -1.35 & 79.1 & 6091.177 & $\mathrm{Ti}_{\mathrm{I}}$ & 2.27 & - & - & -0.44 & 15.9 \\
\hline 5753.622 & $\mathrm{Si}_{\mathrm{I}}$ & 5.61 & - & - & -1.24 & 50.8 & 6092.798 & Ti I & 1.89 & -1.31 & 6.0 & -1.28 & 6.1 \\
\hline 5772.149 & $\mathrm{Si}_{\mathrm{I}}$ & 5.08 & - & - & -1.56 & 57.7 & 6098.694 & Ti I & 3.06 & - & - & -0.16 & 6.1 \\
\hline 5793.080 & $\mathrm{Si}_{\mathrm{I}}$ & 4.93 & -1.91 & 46.0 & -1.94 & 42.6 & 6126.224 & Ti I & 1.07 & - & - & -1.40 & 23.5 \\
\hline 6125.021 & $\mathrm{Si}_{\mathrm{I}}$ & 5.61 & - & - & -1.50 & 34.1 & 6258.104 & Ti I & 1.44 & -1.46 & 54.6 & -0.43 & 52.3 \\
\hline 6131.577 & $\mathrm{Si}_{\mathrm{I}}$ & 5.61 & -1.67 & 25.8 & -1.65 & 26.6 & 4568.345 & $\mathrm{Ti}$ II & 1.22 & -2.85 & 33.6 & -2.85 & 32.3 \\
\hline 6131.858 & $\mathrm{Si}_{\mathrm{I}}$ & 5.61 & -1.66 & 26.6 & -1.64 & 27.0 & 4583.415 & Ti II & 1.16 & -2.85 & 36.2 & -2.84 & 35.1 \\
\hline 6142.494 & $\mathrm{Si} \mathrm{I}_{\mathrm{H}}$ & 5.62 & -1.44 & 37.5 & -1.45 & 36.3 & 4657.209 & Ti II & 1.24 & - & - & -2.31 & 55.8 \\
\hline 6145.020 & $\mathrm{Si} \mathrm{I}_{\mathrm{I}}$ & 5.61 & -1.40 & 40.8 & -1.36 & 41.8 & 4798.539 & Ti II & 1.08 & -2.75 & 45.4 & -2.70 & 44.7 \\
\hline 6243.823 & Si I & 5.61 & -1.22 & 52.5 & -1.19 & 52.7 & 5211.544 & Ti II & 2.59 & -1.59 & 32.1 & -1.54 & 33.2 \\
\hline 6244.476 & Si I & 5.61 & -1.26 & 49.5 & -1.25 & 48.6 & 5336.783 & Ti II & 1.58 & -1.77 & 71.5 & -1.63 & 73.4 \\
\hline 5261.708 & $\mathrm{Ca}_{\mathrm{I}}$ & 2.52 & - & - & -0.65 & 99.4 & 5381.020 & Ti II & 1.57 & -1.91 & 65.1 & -1.95 & 59.5 \\
\hline 5581.979 & $\mathrm{Ca}$ I & 2.52 & - & - & -0.68 & 97.2 & 5418.756 & Ti II & 1.58 & -2.21 & 49.7 & -2.17 & 49.4 \\
\hline 5590.126 & $\mathrm{Ca}$ I & 2.52 & -0.78 & 96.3 & -0.73 & 93.9 & 5657.436 & $\mathrm{~V}_{\mathrm{I}}$ & 1.06 & - & - & - & 9.4 \\
\hline 5867.572 & $\mathrm{Ca}_{\mathrm{I}}$ & 2.93 & -1.62 & 25.3 & -1.59 & 25.1 & 5668.362 & $\mathrm{~V}_{\mathrm{I}}$ & 1.08 & - & 8.7 & - & 6.7 \\
\hline 6161.295 & $\mathrm{Ca}$ I & 2.52 & -1.18 & 71.6 & -1.08 & 69.7 & 5670.851 & $\mathrm{~V}_{\mathrm{I}}$ & 1.08 & - & 21.1 & - & 21.7 \\
\hline 6163.754 & $\mathrm{Ca}$ I & 2.52 & - & - & -1.25 & 83.4 & 5727.661 & $\mathrm{~V}_{\mathrm{I}}$ & 1.05 & - & 10.9 & - & 12.5 \\
\hline 6166.440 & $\mathrm{Ca}$ I & 2.52 & -1.18 & 71.5 & -1.02 & 76.9 & 6090.216 & $\mathrm{~V}_{\mathrm{I}}$ & 1.08 & - & 35.6 & - & 34.4 \\
\hline 6169.044 & $\mathrm{Ca}$ I & 2.52 & -0.75 & 99.4 & -0.70 & 97.0 & 6135.370 & $\mathrm{~V}_{\mathrm{I}}$ & 1.05 & - & 12.6 & - & 11.5 \\
\hline 6169.564 & $\mathrm{Ca}$ I & 2.52 & -0.51 & 117.6 & -0.53 & 119.8 & 6150.154 & $\mathrm{~V}_{\mathrm{I}}$ & 0.30 & - & 12.6 & - & 11.0 \\
\hline 6449.820 & $\mathrm{Ca}$ I & 2.52 & - & - & -0.32 & 127.8 & 6199.186 & $\mathrm{~V}_{\mathrm{I}}$ & 0.29 & - & 15.1 & - & 14.0 \\
\hline 6455.605 & $\mathrm{Ca} \mathrm{I}$ & 2.52 & - & - & -1.43 & 53.7 & 6216.358 & $\mathrm{~V}_{\mathrm{I}}$ & 0.28 & - & - & - & 37.0 \\
\hline 6471.688 & $\mathrm{Ca}$ I & 2.52 & - & - & -0.64 & 101.5 & 6274.658 & $\mathrm{~V}_{\mathrm{I}}$ & 0.27 & - & - & - & 8.7 \\
\hline 6499.654 & $\mathrm{Ca}_{\mathrm{I}}$ & 2.52 & - & - & -0.86 & 87.2 & 6285.165 & $\mathrm{~V}_{\mathrm{I}}$ & 0.28 & - & 10.4 & - & 16.2 \\
\hline 4743.817 & $\mathrm{Sc}_{\mathrm{I}}$ & 1.45 & - & - & - & 8.5 & 4575.092 & Cri & 3.37 & - & - & -0.88 & 13.6 \\
\hline 5356.091 & $\mathrm{Sc}_{\mathrm{I}}$ & 1.86 & - & - & - & 1.8 & 4616.120 & $\mathrm{Cr}_{\mathrm{I}}$ & 0.98 & - & - & -1.31 & 91.9 \\
\hline 5392.075 & $\mathrm{Sc}_{\mathrm{I}}$ & 1.99 & - & - & - & 7.0 & 4626.174 & Cri & 0.97 & - & - & -1.47 & 84.8 \\
\hline 5484.611 & $\mathrm{Sc}_{\mathrm{I}}$ & 1.85 & - & - & - & 3.2 & 4708.019 & Cr I & 3.17 & - & - & -0.06 & 58.1 \\
\hline 5671.826 & $\mathrm{Sc}_{\mathrm{I}}$ & 1.45 & - & - & - & 19.3 & 4737.355 & $\mathrm{Cr} I$ & 3.09 & - & - & -0.06 & 62.0 \\
\hline 6239.408 & $\mathrm{Sc}_{\mathrm{I}}$ & 0.00 & - & - & - & 9.0 & 4756.137 & $\mathrm{Cr}_{\mathrm{I}}$ & 3.10 & 0.09 & 74.6 & 0.03 & 66.4 \\
\hline 5318.346 & Sc II & 1.36 & - & - & - & 18.2 & 4801.047 & Cr I & 3.12 & -0.28 & 51.5 & -0.28 & 46.2 \\
\hline 5357.190 & Sc II & 1.51 & - & 5.4 & - & 5.2 & 4936.335 & Cr I & 3.11 & -0.35 & 48.5 & -0.32 & 47.2 \\
\hline 5526.815 & $\mathrm{Sc}_{\text {II }}$ & 1.77 & - & 77.9 & - & 77.6 & 4964.916 & $\mathrm{Cr} \mathrm{I}_{\mathrm{I}}$ & 0.94 & - & - & -2.50 & 41.6 \\
\hline 5657.874 & Sc II & 1.51 & - & 69.8 & - & 69.2 & 5200.207 & $\mathrm{Cr} \mathrm{I}$ & 3.38 & -0.58 & 24.6 & -0.50 & 26.8 \\
\hline 5684.189 & Sc II & 1.51 & - & 40.8 & - & 41.2 & 5214.144 & Cri & 3.37 & -0.77 & 17.7 & -0.73 & 18.4 \\
\hline 6245.660 & Sc II & 1.51 & - & 38.0 & - & 35.6 & 5238.964 & Cri & 2.71 & -1.43 & 16.6 & -1.36 & 17.9 \\
\hline 6320.867 & Sc II & 1.50 & - & 9.1 & - & 8.4 & 5247.566 & Cr I & 0.96 & -1.73 & 83.1 & -1.61 & 85.1 \\
\hline 4518.023 & Ti I & 0.83 & -0.49 & 76.2 & - & - & 5272.007 & Cr I & 3.45 & - & - & -0.36 & 30.3 \\
\hline 4548.765 & Ti I & 0.83 & -0.55 & 73.7 & - & - & 5287.183 & $\mathrm{Cr} \mathrm{I}_{\mathrm{I}}$ & 3.44 & -0.90 & 12.0 & -0.86 & 12.6 \\
\hline 4562.625 & Ti I & 0.02 & - & - & -2.73 & 11.7 & 5296.691 & Cri & 0.98 & - & - & -1.40 & 93.5 \\
\hline 4617.254 & Ti I & 1.75 & - & - & 0.23 & 64.6 & 5300.751 & $\mathrm{Cr}_{\mathrm{I}}$ & 0.98 & -2.13 & 63.3 & -2.11 & 56.7 \\
\hline 4758.120 & Ti I & 2.25 & 0.44 & 56.0 & 0.26 & 43.3 & 5304.183 & Cri & 3.46 & - & - & -0.72 & 16.0 \\
\hline 4759.272 & Ti I & 2.25 & 0.47 & 58.0 & 0.25 & 45.9 & 5318.810 & $\mathrm{Cr} I$ & 3.44 & -0.66 & 19.3 & -0.65 & 18.8 \\
\hline 4778.259 & Ti I & 2.24 & -0.38 & 18.0 & -0.38 & 17.2 & 5628.621 & Cr I & 3.42 & - & - & -0.82 & 14.6 \\
\hline 4926.147 & Ti I & 0.82 & - & - & -2.17 & 7.0 & 5648.279 & Cri & 3.82 & 0.90 & 5.7 & - & - \\
\hline 5022.871 & Ti I & 0.83 & -0.52 & 77.3 & -0.35 & 79.2 & 5784.976 & Cri & 3.32 & -0.45 & 34.0 & -0.39 & 33.8 \\
\hline 5024.842 & Ti I & 0.82 & -0.66 & 71.5 & -0.48 & 73.8 & 5787.965 & Cri & 3.32 & - & - & -0.12 & 50.4 \\
\hline 5071.472 & Ti I & 1.46 & - & - & -0.77 & 31.3 & 6330.097 & $\mathrm{Cr} \mathrm{I}_{\mathrm{I}}$ & 0.94 & -2.88 & 30.0 & -2.90 & 26.9 \\
\hline 5113.448 & Ti I & 1.44 & -0.86 & 30.3 & -0.88 & 27.6 & 4588.203 & $\mathrm{Cr}_{\text {II }}$ & 4.07 & - & - & -0.73 & 71.7 \\
\hline 5145.464 & Ti I & 1.46 & -0.67 & 38.8 & -0.64 & 37.6 & 4592.049 & $\mathrm{Cr}$ II & 4.07 & -1.30 & 50.1 & -1.23 & 50.8 \\
\hline
\end{tabular}


Table 8. continued.

\begin{tabular}{|c|c|c|c|c|c|c|c|c|c|c|c|c|c|}
\hline \multirow[t]{2}{*}{$\lambda[\AA]$} & \multirow[t]{2}{*}{ Id. } & \multirow{2}{*}{$\begin{array}{c}\chi \\
{[\mathrm{eV}]}\end{array}$} & \multicolumn{2}{|c|}{ Gany 1} & \multicolumn{2}{|c|}{ Gany 2} & \multirow[t]{2}{*}{$\lambda[\AA]$} & \multirow[t]{2}{*}{ Id. } & \multirow{2}{*}{ 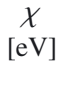 } & \multicolumn{2}{|c|}{ Gany 1} & Gar & \\
\hline & & & $\log g f$ & $E W$ & $\log g f$ & $E W$ & & & & $\log g f$ & $E W$ & $\log g f$ & $E W$ \\
\hline 5305.855 & $\mathrm{Cr}_{\text {II }}$ & 3.83 & - & - & -2.06 & 27.7 & 5491.845 & $\mathrm{Fe}_{\mathrm{I}}$ & 4.19 & -2.19 & 15.2 & -2.23 & 13.8 \\
\hline 5308.377 & Cr II & J & - & - & -1.81 & 27.1 & 74 & $\mathrm{Fe}_{\mathrm{I}}$ & 7 & 1.89 & 31.1 & -1.94 & 27.6 \\
\hline 5313.526 & Cr II & 07 & - & - & -1.61 & 34.9 & 22.454 & $\mathrm{Fe}_{\mathrm{I}}$ & 4.21 & -1.50 & 44.2 & -1.44 & 44.8 \\
\hline 5502.025 & $\mathrm{Cr}_{\text {II }}$ & .17 & - & - & -1.87 & 21.8 & 5560.207 & $\mathrm{Fe}_{\mathrm{I}}$ & 4.43 & -1.12 & 54.3 & -1.09 & 53.0 \\
\hline 4626.538 & $M n_{I}$ & .71 & - & 30.5 & - & 26.8 & 5577.013 & $\mathrm{Fe}_{\mathrm{I}}$ & 5.03 & -1.52 & 11.9 & -1.49 & 12.5 \\
\hline 4739.113 & Mn I & .94 & - & 61.6 & - & 63.3 & 5587.573 & $\mathrm{Fe}_{\mathrm{I}}$ & 4.14 & -1.54 & 45.7 & -1.56 & 41.8 \\
\hline 5004.892 & Mn I & .92 & - & - & - & & & $\mathrm{Fe}_{\mathrm{I}}$ & 4.26 & -1.58 & 37.8 & -1.55 & 37.1 \\
\hline 5394.670 & Mn I & .00 & - & - & - & & & $\mathrm{Fe}_{\mathrm{I}}$ & 3.64 & -2.51 & 22.4 & -2.52 & 21.1 \\
\hline 5399.479 & Mn I & 85 & - & - & - & & & $\mathrm{Fe}_{\mathrm{I}}$ & 4.22 & -0.89 & & -0.79 & 79.4 \\
\hline 5413.684 & Mn I & & - & 240 & - & & & $\mathrm{Fe}_{\mathrm{I}}$ & 4.26 & -1.04 & 67.6 & -0.99 & 66.1 \\
\hline 5420.350 & Mn I & & - & & - & & & & .26 & -2.38 & 9.2 & -2.48 & 5.5 \\
\hline & & & - & & - & & & & & & & & 35.8 \\
\hline & & & - & & - & & & & & -1.79 & & 1.79 & 26.1 \\
\hline 7 & In I & & - & & - & & & & & -1.88 & & -1.81 & 24.6 \\
\hline & $\mathrm{n}_{\mathrm{I}}$ & $3+3$ & - & & - & & & & & -2.34 & $p$ & 2.30 & 12.1 \\
\hline 00 & $\mathrm{Fe}_{\mathrm{I}}$ & 3 & 1.85 & & - & - & & & 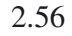 & -2.20 & 2 & -2.13 & 86.6 \\
\hline 676 & $\mathrm{Fe}_{\mathrm{I}}$ & 27 & 2.96 & 1 & - & - & & $\mathrm{Fe}_{\mathrm{I}}$ & 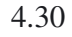 & -1.35 & 0 & -1.44 & 40.8 \\
\hline .925 & $\mathrm{Fe}_{\mathrm{I}}$ & 25 & 2.69 & 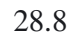 & - & - & 61 & $\mathrm{Fe}_{\mathrm{I}}$ & .26 & -1.13 & 4 & -1.14 & 58.0 \\
\hline & $\mathrm{Fe}_{\mathrm{I}}$ & 6 & -1.57 & 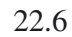 & -1.59 & 21.1 & 738.240 & $\mathrm{Fe}_{\mathrm{I}}$ & 2 & -2.12 & 16.6 & -2.19 & 14.2 \\
\hline & $\mathrm{Fe}_{\mathrm{I}}$ & 3.94 & -2.03 & 2 & -2.00 & 29.1 & & & & -1.21 & 0 & -1.11 & 62.1 \\
\hline 4598.125 & $\mathrm{Fe}_{\mathrm{I}}$ & 2 & -161 & 82 & -1.61 & 76.3 & & $\mathrm{Fe}_{\mathrm{I}}$ & 9 & -3.53 & 00 & -3.47 & 23.7 \\
\hline 4602.000 & $\mathrm{Fe}_{\mathrm{I}}$ & 61 & 332 & 74. & -3.21 & 73.1 & & & .40 & -2.59 & ת & -2.53 & 29.4 \\
\hline 474 & $\mathrm{Fe}_{\mathrm{I}}$ & & -221 & 73 & -2.08 & & & $\mathrm{Fe}_{\mathrm{I}}$ & & -2.40 & & -2.36 & 11.8 \\
\hline & $\mathrm{Fe}_{\mathrm{I}}$ & & 1 & & -1.28 & & & & & & & & 23.4 \\
\hline & $\mathrm{Fe}_{\mathrm{I}}$ & & & & -3.5 & & & & & & & 0 & 15.7 \\
\hline & $\mathrm{Fe}_{\mathrm{I}}$ & & & & -3 & & & & & & & 0 & 9.5 \\
\hline & & & & & -1 & & & & & & & & 43.2 \\
\hline & & & & & -4 . & & & & & & & & 25.3 \\
\hline & $\mathrm{F}$ & & .6 & & -2.5 & & & & & 54 & & & 37.3 \\
\hline & $\mathrm{Fe}$ & & 1.8 & & -1.7 & & & & & 0.70 & 7 & 0 & 74.4 \\
\hline & $\mathrm{Fe}_{1}$ & & 1.56 & & -1.77 & 40 & & & 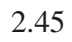 & -2.97 & 57 & -2.89 & 56.8 \\
\hline & $\mathrm{Fe}$ & & 1.72 & 4 & -1.61 & 48 & & & 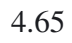 & -1.12 & 43 & -1.05 & 44.5 \\
\hline & & & 2 & & -2.31 & & & & & -1.17 & & 8 & 42.4 \\
\hline & $\mathrm{Fe}$ & & 12 & & -1.2 & & & & & -0.33 & & 6 & 91.2 \\
\hline & $\mathrm{Fe}_{\mathrm{I}}$ & & 08 & & -0.7 & & & & & -4.63 & & & 53.5 \\
\hline 5023 & $\mathrm{Fe}_{\mathrm{I}}$ & & 1.17 & & -1.3 & & & $\mathrm{Fe}_{\mathrm{I}}$ & & -3.50 & 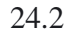 & -3.48 & 23.6 \\
\hline & $\mathrm{Fe}_{\mathrm{I}}$ & & & & -1 & & & & & -0.73 & & 1 & 63.3 \\
\hline & $\mathrm{Fe}$ & & & & & & & & & -3.82 & & 9 & 25.5 \\
\hline & $\mathrm{Fe}$ & & & & & & & & & -0.36 & & 9 & 81.9 \\
\hline & & & & & & & & & & & & & 48.6 \\
\hline & & & & & & & & & & & & & 37.3 \\
\hline & & & . & & & & & & & -1.37 & 32 & & 31.6 \\
\hline & $\Gamma$ & & $3.52-$ & & 3 & 10 & & & & - & 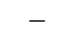 & 5 & 19.5 \\
\hline & Fe & & 5.97 & & -5.8 & & & & & -5.81 & 6 & 6 & 5.8 \\
\hline 65 & $\mathrm{Fe}$ & & -0.90 & & -0.78 & 77 & & & & -2.93 & 72 & -2.83 & 71.2 \\
\hline & $\Gamma$ & & 15 & & -1.50 & 37 & & & & -3.35 & & -3.30 & 50.6 \\
\hline & & & & & & & & & & -1.86 & & & 13.7 \\
\hline & $\mathrm{Fe}$ & & -2.29 & & -2.26 & & & & & -2.95 & 70.3 & -2.93 & 66.1 \\
\hline & $\mathrm{Fe}$ & & -4.8 & & -4.72 & & & & & -1.71 & 48.1 & & 48.4 \\
\hline & $\mathrm{Fe}_{\mathrm{I}}$ & & -1.26 & & -1.16 & & & $\mathrm{Fe}_{\mathrm{I}}$ & 2.56 & -4.25 & 5.8 & -4.34 & 4.3 \\
\hline & $\mathrm{Fe}$ & & 1. & & -1.05 & & & $\mathrm{Fe}_{\mathrm{I}}$ & & -2.45 & 4 & -2.37 & 74.6 \\
\hline & $\mathrm{Fe}$ & & 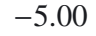 & & $r$. & & & & & & & -2.59 & 82.1 \\
\hline & $\mathrm{Fe}_{\mathrm{I}}$ & & -4.86 & & -4.95 & & & & & -2.52 & & -2.47 & 88.3 \\
\hline & & & & & & & & & & & & & 29.5 \\
\hline & & & & & 12 & & & & & -3.37 & & & 46.6 \\
\hline & & & -3.07 & & -2.84 & & & $\mathrm{~F}$ & & -2.65 & & & 86.8 \\
\hline & $\mathrm{Fe}$ & & 16 & & -1.56 & & & $\mathrm{Fe}$ & 8 & -2.70 & 278 & -2.67 & 27.8 \\
\hline 0.186 & $\mathrm{Fe}$ & & 0.63 & & -0.56 & & & $\mathrm{Fe}$ & 21 & -2.77 & 79.9 & -2.34 & 96.5 \\
\hline & & & 1.73 & & -1.73 & & & $\mathrm{Fc}$ & 46 & -1.66 & 43.9 & -1.67 & 41.2 \\
\hline & & & 176 & & -1.7 & & & $\mathrm{~F}$ & & -2.47 & 77.0 & -2.31 & 79.0 \\
\hline & & & & & & & & & & - & - & -3.79 & 87.8 \\
\hline & $\mathrm{Fe}$ & & - & 42 & -1.31 & 42 & & & & - & - & -1.30 & 53.9 \\
\hline & $\mathrm{Fe}$ & & 10 & 21 & -1.96 & 22.2 & & & 4. & - & - & -1.94 & 9.1 \\
\hline 5483.108 & $\mathrm{Fe}_{\mathrm{I}}$ & 4.15 & -1.46 & 49.1 & -1.45 & 47.1 & 6392.538 & $\mathrm{Fe}_{\mathrm{I}}$ & 2.28 & - & - & -3.98 & 17.3 \\
\hline
\end{tabular}


Table 8. continued.

\begin{tabular}{|c|c|c|c|c|c|c|c|c|c|c|c|c|c|}
\hline$\lambda[\AA]$ & Id. & $\begin{array}{c}\chi \\
{[\mathrm{eV}]}\end{array}$ & $\begin{array}{c}\text { Gany } 1 \\
\log g f\end{array}$ & $\begin{array}{c}\text { Gany } 2 \\
E W\end{array}$ & $\begin{array}{c}\lambda[\AA] \\
\log g f\end{array}$ & $\begin{array}{l}\text { Id. } \\
E W\end{array}$ & 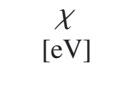 & \multicolumn{2}{|c|}{ Gany 1} & \multicolumn{4}{|l|}{ Gany 2} \\
\hline 6393.612 & $\mathrm{Fe}_{\mathrm{I}}$ & 2.43 & - & - & -1.60 & 132.8 & 5435.866 & $\mathrm{Ni}$ I & 1.99 & -2.47 & 54.2 & -2.38 & 54.1 \\
\hline 6430.856 & $\mathrm{Fe}_{\mathrm{I}}$ & 2.18 & - & - & -2.01 & 117.8 & 5452.860 & $\mathrm{Ni}$ & 3.84 & -1.48 & 17.5 & -1.48 & 18.5 \\
\hline 6498.945 & $\mathrm{Fe}_{\mathrm{I}}$ & 0.96 & - & - & -4.58 & 48.4 & 5494.876 & $\mathrm{Ni}$ & 4.10 & -1.11 & 21.7 & -1.07 & 22.7 \\
\hline 4576.339 & $\mathrm{Fe}_{\text {II }}$ & 2.84 & -3.13 & 66.0 & -3.03 & 66.3 & 5587.853 & $\mathrm{Ni}$ I & 1.93 & -2.37 & 62.4 & -2.32 & 60.4 \\
\hline 4656.981 & $\mathrm{Fe}_{\text {II }}$ & 2.89 & - & - & -3.59 & 41.2 & 5625.312 & $\mathrm{Ni}$ I & 4.09 & -0.59 & 47.6 & -0.63 & 42.9 \\
\hline 4720.149 & $\mathrm{Fe}_{\text {II }}$ & 3.20 & -4.49 & 6.0 & -4.57 & 6.0 & 5628.354 & $\mathrm{Ni}_{\mathrm{I}}$ & 4.09 & - & - & -1.28 & 15.8 \\
\hline 4993.358 & $\mathrm{Fe}_{\text {II }}$ & 2.81 & -3.74 & 40.0 & -3.69 & 40.8 & 5637.128 & $\mathrm{Ni}$ & 4.09 & -0.80 & 36.1 & -0.80 & 34.8 \\
\hline 5197.576 & $\mathrm{Fe}_{\text {II }}$ & 3.23 & -2.45 & 81.4 & -2.32 & 82.3 & 5748.346 & $\mathrm{Ni}$ & 1.68 & -3.26 & 30.7 & -3.22 & 30.5 \\
\hline 5234.630 & $\mathrm{Fe}_{\text {II }}$ & 3.22 & -2.36 & 86.1 & -2.23 & 86.8 & 5846.986 & $\mathrm{Ni}$ & 1.68 & -3.40 & 24.7 & -3.33 & 26.3 \\
\hline 5264.812 & $\mathrm{Fe}_{\text {II }}$ & 3.33 & -2.98 & 52.5 & -2.96 & 50.7 & 6086.276 & $\mathrm{Ni}$ & 4.26 & -0.44 & 47.7 & -0.44 & 45.4 \\
\hline 5325.560 & $\mathrm{Fe}_{\text {II }}$ & 3.22 & -3.16 & 49.0 & -3.15 & 47.3 & 6176.807 & $\mathrm{Ni} \mathrm{I}_{\mathrm{I}}$ & 4.09 & -0.26 & 66.9 & -0.28 & 61.6 \\
\hline 5414.075 & $\mathrm{Fe}_{\text {II }}$ & 3.22 & -3.60 & 29.1 & -3.54 & 31.0 & 6177.236 & $\mathrm{Ni}$ I & 1.83 & -3.44 & 18.4 & -3.52 & 14.8 \\
\hline 5425.257 & $\mathrm{Fe}_{\mathrm{II}}$ & 3.20 & -3.23 & 46.5 & -3.25 & 43.6 & 6186.709 & $\mathrm{Ni}$ & 4.10 & -0.90 & 31.6 & -0.87 & 31.6 \\
\hline 5427.826 & $\mathrm{Fe}_{\text {II }}$ & 6.72 & - & - & -1.31 & 6.7 & 6191.187 & $\mathrm{Ni}$ I & 1.68 & -2.30 & 80.2 & -2.18 & 79.3 \\
\hline 6084.111 & $\mathrm{Fe}_{\mathrm{II}}$ & 3.20 & -3.75 & 24.1 & -3.78 & 22.7 & 6327.604 & $\mathrm{Ni}$ & 1.68 & -3.07 & 41.2 & -3.04 & 39.8 \\
\hline 6149.249 & $\mathrm{Fe}_{\mathrm{II}}$ & 3.89 & -2.76 & 38.5 & -2.73 & 38.6 & 6370.357 & $\mathrm{Ni}$ & 3.54 & - & - & -1.75 & 18.5 \\
\hline 6247.562 & $\mathrm{Fe}_{\text {II }}$ & 3.89 & -2.37 & 56.4 & -2.37 & 53.9 & 6378.256 & $\mathrm{Ni}$ & 4.15 & - & - & -0.77 & 34.3 \\
\hline 6369.463 & $\mathrm{Fe}_{\text {II }}$ & 2.89 & -4.14 & 20.9 & -4.15 & 20.3 & 5218.209 & $\mathrm{Cu}$ & 3.82 & - & 54.1 & - & 55.8 \\
\hline 6383.715 & $\mathrm{Fe}_{\text {II }}$ & 5.55 & - & - & -2.07 & 10.7 & 5220.086 & $\mathrm{Cu}$ & 3.82 & - & 17.3 & - & 17.2 \\
\hline 6385.458 & $\mathrm{Fe}_{\text {II }}$ & 5.55 & - & - & -2.44 & 5.1 & 5782.136 & $\mathrm{Cu}$ & 1.64 & - & 79.9 & - & 85.0 \\
\hline 6416.928 & $\mathrm{Fe}_{\mathrm{II}}$ & 3.89 & -2.65 & 43.5 & -2.69 & 40.5 & 4810.537 & $\mathrm{Zn}$ I & 4.08 & -0.33 & 76.1 & -0.27 & 79.0 \\
\hline 6456.391 & $\mathrm{Fe}_{\mathrm{II}}$ & 3.90 & - & - & -2.24 & 59.4 & 4607.338 & $\mathrm{Sr}_{\mathrm{I}}$ & 0.00 & 0.02 & 48.8 & 0.12 & 48.1 \\
\hline 4749.662 & Co I & 3.05 & - & 50.0 & - & 40.0 & 4883.690 & $Y_{\text {II }}$ & 1.08 & - & - & 0.06 & 62.8 \\
\hline 4792.862 & CoI & 3.25 & - & 34.9 & - & 34.0 & 4900.124 & $Y_{\text {II }}$ & 1.03 & -0.29 & 55.0 & -0.07 & 60.1 \\
\hline 4813.479 & Co I & 3.21 & - & 48.0 & - & 48.6 & 5087.426 & $Y_{\text {II }}$ & 1.08 & -0.43 & 46.9 & -0.33 & 48.5 \\
\hline 5212.691 & Co I & 3.51 & - & 18.4 & - & 20.4 & 5200.415 & $Y_{\text {II }}$ & 0.99 & -0.70 & 39.0 & -0.71 & 36.5 \\
\hline 5280.629 & CoI & 3.63 & - & - & - & 20.6 & 5289.820 & $Y_{\text {II }}$ & 1.03 & -1.77 & 5.4 & - & - \\
\hline 5342.708 & Co I & 4.02 & - & 32.1 & - & 32.1 & 5402.780 & $\mathrm{Y}_{\text {II }}$ & 1.84 & -0.48 & 15.6 & -0.61 & 15.0 \\
\hline 5359.192 & Co I & 4.15 & - & 11.0 & - & 10.4 & 4739.454 & $\mathrm{Zr} \mathrm{I}_{\mathrm{I}}$ & 0.65 & 0.00 & 7.5 & 0.04 & 7.9 \\
\hline 5381.772 & $\mathrm{CoI}$ & 4.2 & - & 9.9 & - & 6.0 & 4613 & $\mathrm{Zr}$ II & 0.97 & -0.62 & 37.1 & -0.61 & 35.7 \\
\hline 5454.572 & Co I & 4.07 & - & - & - & 18.2 & 5112.279 & $\mathrm{Zr}$ II & 1.66 & -0.75 & 10.2 & -0.81 & 12.3 \\
\hline 5647.234 & Co I & 2.2 & - & 15.1 & - & 14.8 & 5853.688 & Ba II & 0.60 & -1.01 & 64.0 & -0.84 & 65.6 \\
\hline 6000.678 & Co I & 3.62 & - & 4.3 & - & 5.8 & 6141.727 & Ba II & 0.70 & 0.13 & 120.0 & 0.24 & 119.6 \\
\hline 6455.001 & Co I & 3.63 & - & - & - & 14.4 & 6496.908 & $\mathrm{Ba}$ II & 0.60 & -0.05 & 101.0 & -0.07 & 106.2 \\
\hline 4935.831 & $\mathrm{Ni}$ & 3.94 & -0.41 & 64.4 & -0.37 & 72.0 & 4523.080 & $\mathrm{Ce}_{\text {II }}$ & 0.52 & 0.24 & 21.4 & - & - \\
\hline 4946.029 & $\mathrm{Ni}$ I & 3.80 & -1.18 & 30.5 & -1.22 & 27.5 & 4562.367 & $\mathrm{Ce}_{\text {II }}$ & 0.48 & 0.37 & 27.9 & - & - \\
\hline 4953.200 & $\mathrm{Ni}$ I & 3.74 & -0.82 & 51.8 & -0.67 & 56.1 & 4628.160 & $\mathrm{Ce}_{\text {II }}$ & 0.52 & 0.21 & 20.4 & 0.27 & 21.8 \\
\hline 5010.934 & $\mathrm{Ni}$ I & 3.63 & -0.92 & 51.8 & -0.91 & 49.1 & 4773.959 & $\mathrm{Ce}_{\text {II }}$ & 0.92 & 0.32 & 12.7 & 0.31 & 12.1 \\
\hline 5032.723 & $\mathrm{Ni}$ I & 3.90 & -1.20 & 25.6 & -1.09 & 28.8 & 5274.236 & Ce II & 1.04 & 0.48 & 14.3 & 0.40 & 12.0 \\
\hline 5094.406 & $\mathrm{Ni}$ I & 3.83 & -1.14 & 31.3 & -1.06 & 33.1 & 5089.831 & $\mathrm{Nd}_{\text {II }}$ & 0.20 & -1.31 & 2.7 & -1.23 & 3.5 \\
\hline 5197.157 & $\mathrm{Ni} \mathrm{I}_{\mathrm{I}}$ & 3.90 & -1.15 & 27.8 & -1.09 & 28.8 & 5319.820 & $\mathrm{Nd}_{\text {II }}$ & 0.55 & -0.21 & 14.6 & -0.17 & 15.1 \\
\hline 5220.300 & $\mathrm{Ni}$ I & 3.74 & -1.30 & 27.6 & -1.23 & 29.4 & 4566.233 & Sm II & 0.33 & -0.19 & 12.3 & -0.19 & 12.0 \\
\hline 5392.330 & $\mathrm{Ni}$ I & 4.15 & -1.24 & 15.7 & -1.31 & 13.3 & & & & & & & \\
\hline
\end{tabular}

Notes. Oscillator strengths $(\log g f)$ and raw $E W \mathrm{~s}$ (before the conversion set out by Eqs. (1) and (2)), given in mÅ, of both the Ganymede spectra observed in the first (Gany 1) and second (Gany 2) runs are listed (except for C, for which the analysis is based on spectral synthesis). Lines with missing $g f$ values represent the elements with hyperfine structure $(\mathrm{Mg}, \mathrm{Sc}, \mathrm{V}, \mathrm{Mn}, \mathrm{Co}$, and $\mathrm{Cu})$ and the detailed line splitting is shown in Table 9 .

3- The consideration of the $[\mathrm{X} / \mathrm{Fe}]$ ratios with age revealed much differing behaviour of the elements, suggesting that the age-metallicity relation has more underlying complexity than commonly recognised. The $[\mathrm{Mg} / \mathrm{Fe}],[\mathrm{Sc} / \mathrm{Fe}]$, and $[\mathrm{Ti} / \mathrm{Fe}]$ decrease towards younger ages. The $[\mathrm{Cu} / \mathrm{Fe}]$ and $[\mathrm{Mn} / \mathrm{Fe}]$ ratios initially increase towards younger stars up to the solar age, and then decrease towards the youngest objects, a result that may speak of differing yields in the SN Ia production of these elements, related to metallicity and age, as well as a possible influence of the evolution with time of the weak s-process yields, operating off of massive stars. The steepest relation with age was found for the $[\mathrm{Ba} / \mathrm{Fe}]$ ratio, but only for ages younger than the solar one, and a similar but less evident behaviour is seen for $\mathrm{Zr}$, Ce, and $\mathrm{Nd}$. Other heavy s-process elements, however, such as $\mathrm{Sr}$ and $\mathrm{Y}$, show a linearly increasing $[\mathrm{X} / \mathrm{Fe}]$ towards younger ages, particularly clearer for $\mathrm{Sr}$. [Sm/Fe] significantly decreases for stars younger than the Sun. Thus, the $[\mathrm{Cu} / \mathrm{Ba}]$ ratio clearly decreases towards younger stars, and the same is seen at a significant level for $[\mathrm{Sm} / \mathrm{Ba}]$.

4- Considering the average of elements with similar behaviour with age considerably reinforces the aforementioned results, particularly for the $[\mathrm{Sc}, \mathrm{Ti} / \mathrm{Fe}],[\mathrm{Mn}, \mathrm{Cu} / \mathrm{Fe}],[\mathrm{Sr}, \mathrm{Y} / \mathrm{Fe}]$, and $[\mathrm{Zr}, \mathrm{Ba}, \mathrm{Nd} / \mathrm{Fe}]$ relations.

5- The consideration of element ratios not directly involving Fe shows some marked behaviour. Particularly, the $[\mathrm{Ba} / \mathrm{Mg}]$, $[\mathrm{Ba} / \mathrm{Zn}],[\mathrm{Ba} / \mathrm{Sm}],[\mathrm{Sr} / \mathrm{Sm}]$, and $[\mathrm{Y} / \mathrm{Sm}]$ steeply increase 
Table 9. Oscillator strengths $(\log g f)$ for lines with hyperfine structure computed based on the Ganymede spectra observed in the first (Gany 1$)$ and second (Gany 2) runs.

\begin{tabular}{|c|c|c|c|c|c|c|c|c|c|c|c|c|c|c|}
\hline$\lambda[\AA]$ & \multicolumn{2}{|c|}{$\begin{array}{c}\log g f \\
\text { Gany } 1 \text { Gany } 2\end{array}$} & $\lambda[\AA ̊]$ & \multicolumn{2}{|c|}{$\begin{array}{c}\log g f \\
\text { Gany } 1 \text { Gany } 2\end{array}$} & $\lambda[\AA ̊]$ & \multicolumn{2}{|c|}{$\log g f$} & $\lambda[\AA]$ & \multicolumn{2}{|c|}{$\log g f$} & $\lambda[\AA ̊]$ & \multicolumn{2}{|c|}{$\begin{array}{c}\log g f \\
\text { Gany } 1 \text { Gany } 2\end{array}$} \\
\hline \multicolumn{3}{|c|}{ Mg І: 4571.102} & Sc II: & :5657.8 & & & 5274. & & & 553 & & Co I: & : 5381 & \\
\hline 1.078 & -6.67 & -6.49 & 7.808 & -1.33 & -1.32 & 640 & - & 9 & 691 & -2.76 & -2.79 & 81.695 & -0.47 & -0.69 \\
\hline & -6.71 & & 57.841 & -1.23 & & & - & & & -2.64 & & & & \\
\hline 096 & -5.81 & 63 & 57.874 & -1.44 & -1.43 & 76 & - & & & -2.61 & & .772 & -0.63 & -0.85 \\
\hline Mg I: & : 4730.0 & & 5657.893 & -1.33 & -1.32 & & 6285 & & 764 & -2.64 & & 1.799 & -0.74 & -0.96 \\
\hline 0.031 & -3.13 & -3.10 & Sc пा: & : 5684.1 & & & -2.11 & -1.86 & 37.802 & -2.28 & & 81.824 & -0.69 & -0.91 \\
\hline & -3.17 & -3.14 & 84.123 & -1.72 & -1.71 & 628 & -2.11 & -1.86 & Mr & I: 6013. & & Co I: & : 5454 & \\
\hline 0.046 & -2.27 & -2.24 & 5684.156 & -1.62 & -1.61 & 6285.183 & -2.11 & & 174 & -0.75 & -0.67 & .495 & - & -0.38 \\
\hline Mg I: & : 5711.0 & & 5684.189 & -1.83 & -1.82 & Mi & I: 4626. & & & -0.96 & & & - & \\
\hline 074 & -2.66 & -2.69 & 5684.208 & -1.72 & & 64 & -0.94 & -0 & & -1.10 & & & - & \\
\hline & -2.7 & & Sc пा: & : 6245.6 & & & -0.14 & & & & & & - & \\
\hline & -1.8 & -1.83 & 6245 & -1.78 & -1.81 & & -0.39 & & & -1.35 & & 4.624 & - & \\
\hline Sc г: & $: 474$ & & & -1.89 & -1.92 & & -0.19 & & & I: 602 & & Co I: & 5647 & \\
\hline 751 & - & -0.37 & & -1.68 & & & -0.49 & & 764 & -1.37 & -1 & .191 & & -2 \\
\hline & - & -0.28 & 576 & -1.77 & -1.80 & & I: 4739. & & & -1.22 & & & & \\
\hline & - & -0.49 & Sc II: & : 632 & & 99 & -1.30 & & & -0.39 & & & & \\
\hline 36 & - & -0.38 & .884 & -2 & & & -1 & & & -0.60 & & 261 & & \\
\hline Sc г: & 356. & & & -2.63 & & & -1 & & & -0.47 & & .291 & -2 & \\
\hline .025 & - & -0.67 & 332 & -2.42 & -2.47 & & -1 . & & & I: 4749. & & Сo I: & $: 60$ & \\
\hline & - & -0.58 & 799 & -2.52 & -2.57 & 473 & -2.50 & & & -0.63 & & .607 & & -2.09 \\
\hline & - & -0.79 & V I: & 5657.4 & & Mi & I: $\mathbf{5 0 0}$ & & & -0.81 & & & & \\
\hline & - & -0.68 & 418 & - & -1 . & 78 & - & -2 & & -0.92 & & & & \\
\hline & 202 & & & - & -1 & & - & & & -0.88 & & & & \\
\hline $00 c^{2}-2-2$ & - & 0.04 & & - & -1 & & - & & & -2.28 & & .752 & & \\
\hline & - & & & 5668 & & & - & & & I: 4792. & & & & \\
\hline & - & & 344 & -1 & 1 & & - & & & -2 & & & - & \\
\hline & - & & & -1 & & & 53 & & & -1 & & & - & \\
\hline Sc г: & 104 & & 380 & -1 & -1 & & - & & & -0 & & & - & \\
\hline & - & -0.45 & $V_{\text {I: }}$ & 56 & & & - & & & -0 & & & - & \\
\hline & - & .36 & & -0 & -0 & & - & & 364 & -0.50 & & .044 & - & \\
\hline & - & -0.57 & 51 & -0.93 & -0 & & - & & & I: 4813. & & Cu I: & & \\
\hline & - & -0.46 & 369 & -0.93 & -0.97 & 03 & - & & & & & & & \\
\hline Sc г: & 671 & & $V_{\text {I: }}$ & 572 & & Mı & 520 & & & & & & & \\
\hline & - & 017 & & & & & - & -0 & & & & & & \\
\hline & - & & & & & & - & & & & & & & \\
\hline & - & & & & & & - & & & & & & & \\
\hline & - & & & 609 & & & - & & & $=0$ & & & -0.65 & \\
\hline & 239 & & & -0 . & & 36 & - & & & & & .074 & -0 & \\
\hline & - & & & -0 & & & & & & & & $\mathrm{Cu}$ : & & \\
\hline & - & -1 & & -0 & & & - & & & & & & & \\
\hline & - & 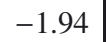 & & 613 & & & & & & & & & & \\
\hline & - & 3 & & -1 & -1 & & & & 759 & -0.99 & & & & \\
\hline Sc пі: & 398 & & & -1 & -1 & & -0 & & & : 528 & & & & \\
\hline & - & -2.30 & 388 & -1 & -1.27 & 722 & -1.3 & -1 & & - & & & -1.41 & -1.40 \\
\hline & - & -2.20 & V I: & 615 & & & $=0$ & & & - & & 92 & -1.41 & -1.40 \\
\hline & - & -2.41 & & -1.99 & -2.03 & & -2.3 & & & - & & 220.095 & -1.09 & -1.08 \\
\hline 8.365 & - & -2.30 & 6150.154 & -1.99 & -2.03 & & -2.2 & -2 & & - & & Cu I: & & \\
\hline Sc II: & : 5357 & & 6150.172 & -1.99 & -2.03 & & -3.10 & -3.02 & 280.672 & - & -1.10 & 032 & -3.58 & \\
\hline & -2.71 & -2.76 & & 6199 & & & -2.00 & -1.92 & & I: $\mathbf{5 3 4 2 . 7}$ & & 42 & -3.89 & -3.79 \\
\hline & 2.6 & -2.66 & & -1 & -1 & 429 & -1.91 & -1.83 & & -0.12 & & & -3.19 & -3.09 \\
\hline & 2.8 & -2.87 & & -1 . & & & I: 54 & & & -0.19 & & & -3 & \\
\hline .207 & -2.7 & -2.76 & 99.204 & -1.91 & & & -4 & & & -0 & & & & \\
\hline & & & & & & & & & & & & & & \\
\hline & & ( & & - & -1 & & $-4 .$. & & .776 & -0.33 & & & & -3.09 \\
\hline & 0.8 & -0.85 & & - & -1 & & -4.7 & -4 & & :535 & & & -3.19 & -3.09 \\
\hline & -1.08 & -1.06 & 6216.376 & - & -1 . & 5432.598 & -4.80 & -4.74 & 359.115 & -0.51 & -0.53 & 5782.113 & -2.84 & -2.74 \\
\hline 5526.834 & -0.97 & -0.95 & & & & & & 5359.158 & -0.59 & -0.61 & 5782.124 & -2.84 & -2.7 & \\
\hline & & & & & & & & -0.67 & -0.69 & 782.15 & & -2.64 & & \\
\hline & & & & & & & & -0 & -0.80 & 782.173 & -2.39 & -2.29 & & \\
\hline & & & & & & & 59.244 & -0.73 & -0.75 & & & & & \\
\hline
\end{tabular}


Table 10. Elemental abundance relative to iron.

\begin{tabular}{|c|c|c|c|c|c|c|c|c|c|c|}
\hline Star & {$[\mathrm{Na} / \mathrm{Fe}]$} & {$[\mathrm{Mg} / \mathrm{Fe}]$} & {$[\mathrm{Si} / \mathrm{Fe}]$} & {$[\mathrm{Ca} / \mathrm{Fe}]$} & {$[\mathrm{Sc} / \mathrm{Fe}]$} & {$[\mathrm{Ti} / \mathrm{Fe}]$} & {$[\mathrm{V} / \mathrm{Fe}]$} & {$[\mathrm{Cr} / \mathrm{Fe}]$} & {$[\mathrm{Mn} / \mathrm{Fe}]$} & {$[\mathrm{Co} / \mathrm{Fe}]$} \\
\hline HD 1835 & 0.04 & 0.00 & -0.01 & 0.08 & -0.01 & -0.02 & 0.08 & 0.04 & 0.04 & -0.03 \\
\hline HD 20807 & 0.05 & 0.02 & 0.00 & 0.00 & 0.06 & 0.04 & 0.04 & -0.02 & -0.06 & 0.05 \\
\hline HD 26491 & -0.02 & 0.06 & 0.02 & 0.03 & 0.06 & 0.04 & 0.07 & 0.02 & 0.00 & 0.03 \\
\hline HD 33021 & -0.05 & 0.14 & 0.08 & 0.12 & 0.11 & 0.16 & 0.11 & -0.01 & -0.18 & 0.06 \\
\hline HD 39587 & -0.10 & -0.01 & -0.04 & 0.09 & 0.01 & 0.03 & -0.03 & 0.03 & -0.06 & -0.05 \\
\hline HD 43834 & 0.00 & 0.05 & 0.00 & -0.02 & 0.02 & -0.02 & 0.04 & -0.01 & 0.07 & 0.02 \\
\hline HD 50806 & 0.04 & 0.21 & 0.09 & 0.05 & 0.06 & 0.09 & 0.08 & 0.00 & -0.04 & 0.08 \\
\hline HD 53705 & 0.03 & 0.16 & 0.06 & 0.05 & 0.13 & 0.11 & 0.07 & 0.02 & -0.12 & 0.09 \\
\hline HD 84117 & 0.08 & -0.03 & 0.03 & -0.03 & -0.01 & -0.05 & 0.03 & -0.02 & -0.09 & -0.04 \\
\hline HD 102365 & - & 0.09 & 0.03 & 0.09 & 0.06 & 0.11 & 0.05 & -0.02 & -0.11 & 0.05 \\
\hline HD 112164 & 0.08 & 0.07 & 0.04 & -0.07 & -0.04 & -0.04 & 0.05 & -0.01 & 0.06 & 0.05 \\
\hline HD 114613 & 0.08 & 0.04 & 0.02 & -0.02 & -0.01 & 0.00 & 0.02 & 0.00 & 0.08 & 0.01 \\
\hline HD 115383 & -0.08 & 0.01 & -0.03 & -0.03 & 0.02 & 0.00 & 0.04 & -0.03 & -0.01 & -0.02 \\
\hline HD 115617 & -0.04 & 0.06 & 0.00 & 0.00 & 0.05 & 0.02 & 0.01 & -0.01 & 0.03 & -0.02 \\
\hline HD 117176 & -0.10 & 0.07 & 0.01 & 0.02 & 0.03 & 0.01 & 0.04 & -0.01 & -0.05 & 0.02 \\
\hline HD 128620 & 0.06 & 0.04 & 0.01 & -0.06 & 0.03 & 0.01 & 0.07 & -0.01 & 0.07 & 0.07 \\
\hline HD 141004 & -0.05 & 0.02 & -0.01 & -0.01 & 0.04 & 0.03 & 0.04 & -0.03 & -0.04 & 0.06 \\
\hline HD 146233 & -0.03 & 0.00 & 0.03 & 0.02 & 0.07 & -0.01 & 0.07 & 0.01 & -0.01 & -0.02 \\
\hline HD 147513 & - & -0.06 & -0.04 & 0.03 & 0.05 & 0.07 & 0.12 & -0.02 & -0.07 & -0.03 \\
\hline HD 160691 & 0.09 & 0.07 & 0.03 & -0.06 & 0.00 & -0.02 & 0.07 & -0.03 & 0.07 & 0.03 \\
\hline HD 177565 & 0.02 & 0.04 & 0.01 & -0.01 & -0.04 & 0.01 & 0.03 & 0.02 & 0.05 & 0.01 \\
\hline HD 181321 & - & -0.06 & 0.03 & 0.03 & -0.07 & 0.01 & - & 0.03 & -0.13 & 0.11 \\
\hline HD 188376 & -0.03 & 0.03 & 0.02 & 0.03 & -0.07 & -0.07 & 0.03 & 0.00 & 0.01 & -0.06 \\
\hline HD 189567 & -0.03 & 0.11 & 0.04 & 0.00 & 0.10 & 0.11 & 0.03 & 0.01 & -0.12 & 0.03 \\
\hline \multirow[t]{2}{*}{ HD 196761} & 0.00 & 0.06 & 0.02 & -0.01 & 0.06 & 0.05 & -0.01 & 0.01 & -0.02 & 0.05 \\
\hline & {$[\mathrm{Ni} / \mathrm{Fe}]$} & {$[\mathrm{Cu} / \mathrm{Fe}]$} & {$[\mathrm{Zn} / \mathrm{Fe}]$} & {$[\mathrm{Sr} / \mathrm{Fe}]$} & {$[\mathrm{Y} / \mathrm{Fe}]$} & {$[\mathrm{Zr} / \mathrm{Fe}]$} & {$[\mathrm{Ba} / \mathrm{Fe}]$} & {$[\mathrm{Ce} / \mathrm{Fe}]$} & {$[\mathrm{Nd} / \mathrm{Fe}]$} & {$[\mathrm{Sm} / \mathrm{Fe}]$} \\
\hline HD 1835 & -0.02 & -0.01 & 0.07 & 0.15 & 0.02 & 0.09 & 0.19 & 0.02 & - & -0.29 \\
\hline HD 20807 & -0.03 & 0.02 & -0.03 & 0.04 & -0.08 & 0.04 & -0.06 & 0.07 & 0.02 & 0.15 \\
\hline HD 26491 & 0.00 & 0.03 & 0.02 & -0.08 & -0.08 & -0.07 & 0.03 & -0.06 & -0.11 & -0.08 \\
\hline HD 33021 & -0.02 & -0.03 & -0.03 & -0.06 & -0.13 & 0.13 & 0.02 & -0.03 & 0.06 & -0.02 \\
\hline HD 39587 & -0.08 & -0.11 & -0.01 & 0.19 & 0.06 & 0.16 & 0.33 & 0.16 & - & -0.14 \\
\hline HD 43834 & 0.03 & 0.03 & 0.02 & 0.06 & -0.04 & 0.07 & -0.03 & 0.00 & -0.06 & 0.06 \\
\hline HD 50806 & 0.04 & 0.15 & 0.10 & -0.08 & -0.06 & 0.11 & 0.00 & 0.01 & -0.09 & 0.10 \\
\hline HD 53705 & -0.02 & 0.05 & 0.13 & -0.09 & -0.15 & 0.00 & -0.02 & -0.08 & -0.07 & -0.13 \\
\hline HD 84117 & -0.04 & -0.03 & -0.03 & 0.09 & 0.07 & 0.15 & 0.15 & -0.02 & 0.09 & 0.00 \\
\hline HD 102365 & -0.02 & 0.02 & 0.07 & -0.02 & -0.06 & -0.10 & 0.12 & -0.06 & 0.04 & 0.02 \\
\hline HD 112164 & 0.03 & 0.14 & 0.00 & -0.06 & -0.16 & -0.06 & -0.03 & -0.09 & -0.09 & -0.10 \\
\hline HD 114613 & 0.03 & 0.10 & 0.02 & -0.02 & -0.06 & -0.01 & 0.00 & -0.14 & -0.11 & -0.02 \\
\hline HD 115383 & -0.03 & 0.05 & -0.07 & 0.00 & 0.01 & 0.06 & 0.20 & -0.04 & 0.16 & 0.02 \\
\hline HD 115617 & -0.01 & -0.01 & 0.08 & 0.05 & -0.11 & 0.01 & -0.02 & 0.05 & -0.07 & 0.28 \\
\hline HD 117176 & 0.00 & 0.07 & -0.06 & -0.03 & -0.06 & 0.04 & 0.06 & 0.06 & 0.19 & 0.11 \\
\hline HD 128620 & 0.04 & 0.14 & -0.08 & - & -0.06 & 0.06 & -0.08 & -0.06 & -0.01 & 0.04 \\
\hline HD 141004 & -0.01 & 0.10 & -0.05 & -0.02 & -0.02 & -0.05 & 0.03 & -0.07 & - & -0.10 \\
\hline HD 146233 & -0.01 & -0.02 & -0.04 & 0.17 & 0.00 & 0.06 & 0.12 & 0.02 & 0.13 & 0.09 \\
\hline HD 147513 & -0.06 & -0.11 & -0.08 & - & 0.22 & 0.24 & 0.43 & 0.06 & 0.18 & - \\
\hline HD 160691 & 0.03 & 0.18 & 0.11 & 0.01 & -0.06 & 0.07 & 0.01 & -0.08 & -0.09 & 0.09 \\
\hline HD 177565 & 0.04 & 0.04 & 0.02 & -0.06 & -0.04 & -0.09 & -0.01 & 0.02 & -0.10 & 0.07 \\
\hline HD 181321 & -0.09 & - & -0.14 & - & -0.04 & - & 0.23 & - & - & - \\
\hline HD 188376 & -0.02 & -0.03 & -0.10 & - & 0.00 & 0.04 & 0.21 & -0.07 & 0.20 & -0.19 \\
\hline HD 189567 & 0.00 & -0.04 & 0.03 & -0.07 & -0.07 & 0.03 & 0.06 & 0.08 & 0.06 & 0.14 \\
\hline HD 196761 & 0.00 & 0.01 & 0.08 & -0.09 & -0.16 & 0.05 & -0.03 & -0.04 & -0.04 & 0.26 \\
\hline
\end{tabular}

Notes. Our results for carbon abundances are presented in Table 6.

towards younger ages for stars younger than the Sun. Also, the $[\mathrm{Sr} / \mathrm{Mg}]$ and $[\mathrm{Y} / \mathrm{Mg}]$ ratios linearly increase towards younger ages, and the same is seen for $[\mathrm{Sr} / \mathrm{Zn}]$ and $[\mathrm{Y} / \mathrm{Zn}]$.

6- Possible correlations involving the abundances, the condensation temperatures of the different elements, and the presence of exoplanets in our programme stars were deeply investigated, but no significant correlation was found.

The detailed consideration of precise element abundances derived from high-quality atmospheric parameters and spectroscopic data, tied to masses, kinematics, and ages for solartype stars, generally provides a wealth of interesting data, contributing towards a broader understanding of the evolution of the Galaxy in its chemical and dynamical aspects.

Acknowledgements. R.D.S. thanks the financial support from the Coordenação de Aperfeiçoamento de Pessoal de Nível Superior (CAPES) in the form of a fellowship (PROAP/INPE). L.S.R. thanks the grants (100454/2004-6 and 309326/2009-5) received from the Brazilian Foundation CNPq, and also Luzia P. Rité and Charles Rité for their help in data reductions. G.F.P.M. acknowledges the financial support by CNPq (476909/2006-6 and 474972/2009-7) and FAPERJ (APQ1/26/170.687/2004) grants. This research has made use of the SIMBAD database, operated at CDS, Strasbourg, France, and of NASA's Astrophysics Data System. We acknowledge many fruitful discussions with Verne V. Smith and Katia Cunha. We all thank the critical and important report from the referee Dr. Gustafsson, which has deeply improved this manuscript. 


\section{References}

Allen, D. M., \& Porto de Mello, G. F. 2011, A\&A, 525, 63 Asplund, M. 2005, ARA\&A, 43, 481

Batten, A. H., Fletcher, J. M., \& MacCarthy, D. G. 1989, in Eighth Catalogue of the Spectroscopic Binary Systems, PDAO, 17, 1

Bensby, T., Feltzing, S., Lundström, I., \& Ilyn, I. 2005, A\&A, 433, 185

Bensby, T., Zenn, A. R., Oey, M. S., \& Feltzing, S. 2007, ApJ, 663, L13

Castro, S., Porto de Mello, G. F., \& da Silva, L. 1999, MNRAS, 305, 693

Chen, Y. Q., Nissen, P. E., Zhao, G., Zhang, H. W., \& Benoni, T. 2000, A\&AS, 141,491

Chen, Y. Q., Zhao, G., Izumiura, H., et al. 2008, AJ, 135, 618

Crawford, D. L. 1975, AJ, 80, 955

Crawford, D. L., Barnes, J. V., Faure, B. Q., \& Golson, J. C. 1966, AJ, 71, 709

da Silva, R., Milone, A. C., \& Reddy, B. E. 2011, A\&A, 526, A71

Dehnen, W., \& Binney, J. J. 1998, MNRAS, 298, 387

del Peloso, E. F., Cunha, K., da Silva, L., \& Porto de Mello, G. F. 2005, A\&A, 441,1149

Díaz-Cordovés, J., Claret, A., \& Giménez, A. 1995, A\&AS, 110, 329

Duquennoy, A., \& Mayor, M. 1991, A\&A, 248, 485

Edvardsson, B., Andersen, J., Gustafsson, B., et al. 1993, A\&A, 275, 101

ESA 1997, The Hipparcos and Tycho Catalogues, ESA SP-1200

Everitt, B. S., Landau, S., Leese, M., \& Stahl, D. 2001, in Cluster Analysis, 5th edn. (London: Wiley), 77

Fabregat, J., \& Reglero, V. 1990, A\&AS, 82, 531

Feltzing, S., Fohlman, M., \& Bensby, T. 2007, A\&A, 467, 665

Ferro, A. A., Parrao, L., Schuster, W., et al. 1990, A\&AS, 83, 225

Flower, P. J. 1996, ApJ, 469, 355

Fuhrmann, K. 2004, Astron. Nachr., 325, 3

Grevesse, N., \& Noels, A. 1993, in Origin and Evolution of the Elements (Cambridge University Press)

Gronbech, B., \& Olsen, E. H. 1976, A\&AS, 25, 213

Gronbech, B., \& Olsen, E. H. 1977, A\&AS, 27, 443

Guenther, D. B., \& Demarque, P. 1997, ApJ, 484, 937

Hoffleit, D., \& Jaschek, C. 1982, in The Bright Star Catalogue, Yale University Observatory, New Haven

Holberg, J. B., Oswalt, T. D., \& Sion, E. M. 2002, ApJ, 571, 512

Holmberg, J., Nordström, B., \& Andersen, J. 2007, A\&A, 475, 519

Holmberg, J., Nordström, B., \& Andersen, J. 2009, A\&A, 501, 941

Johnson, D. R. H., \& Soderblom, D. R. 1987, AJ, 93, 864

Kim, Y.-C., Demarque, P., Yi, S. K., \& Alexander, D. R. 2002, ApJS, 143, 499

König, B., Fuhrmann, K., Neuhäuser, R., Charbonneau, D., \& Jayawardhana, R. 2002, A\&A, 394, L43

Korotin, S., Mishenina, T., Gorbaneva, T., \& Soubiran, C. 2011, MNRAS, 415, 2093

Kupka, F., Piskunov, N. E., Ryabchikova, T. A., Stempels, H. C., \& Weiss, W. W. 1999, A\&AS, 138, 119

Kupka, F., Ryabchikova, T. A., Piskunov, N. E., Stempels, H. C., \& Weiss, W. W. 2000, BaltA, 9, 590

Kurucz, R. L. 1992, Rev. Mex. Astron. Astrofis., 23, 45

Kurucz, R. L., Furenlid, I., Brault, J., \& Testerman, L. 1984, in The Solar Flux Atlas from $296 \mathrm{~nm}$ to $1300 \mathrm{~nm}$, National Solar Observatory
Lodders, K. 2003, ApJ, 591, 1220

Lodders, K., Palme, H., \& Gail, H.-P. 2009, in Abundance of the Elements in the Solar System, Landolt Börnstein, New Series, Vol. VI/4B, Chap. 4.4, ed. J. E. Trümper (Berlin, Heidelberg, New York: Springer-Verlag), 560

López-Santiago, J., Montes, D., Gálvez-Ortiz, M. C., et al. 2010, A\&A, 514, A97

Lyra, W., \& Porto de Mello, G. F. 2005, A\&A, 431, 329

Mason, B. D., Wycoff, G. L., Hartkopf, W. I., Douglass, G. G., \& Worley, C. E. 2001, AJ, 122, 3466

McWilliam, A. 1997, ARA\&A, 35, 503

Meylan, T., Furenlid, I., Wiggs, M. S., \& Kurucz, R. L. 1993, ApJS, 85, 163

Montes, D., López-Santiago, J., Gálvez, M. C., et al. 2001, MNRAS, 328, 45

Moore, C.E., Minnaert, M., \& Houtgast, J. 1966, in The Solar Spectrum $2935 \AA$ to $8770 \AA$, Nat. Bur. Std., US Monograph 61

Neves, V., Santos, N. C., Sousa, S. G., Correia, A. C. M., \& Israelian, G. 2009, A\&A, 497, 563

Olsen, E. H. 1977, A\&AS, 29, 313

Olsen, E. H. 1983, A\&AS, 54, 55

Olsen, E. H. 1993, A\&AS, 102, 89

Olsen, E. H. 1994a, A\&AS, 104, 429

Olsen, E. H. 1994b, A\&AS, 106, 257

Olsen, E. H., \& Perry, C. L. 1984, A\&AS, 56, 229

Perry, C. L., Olsen, E. H., \& Crawford, D. L. 1987, PASP, 99, 1184

Piskunov, N. E., Kupka, F., Ryabchikova, T. A., Weiss, W. W., \& Jeffery, C. S. 1995, A\&AS, 112, 525

Porto de Mello, G. F., \& da Silva, L. 1997a, ApJ, 476, L89

Porto de Mello, G. F., \& da Silva, L. 1997b, ApJ, 482, L89

Porto de Mello, G. F., Lyra, W., \& Keller, G. R. 2008, A\&A, 488, 653

Raghavan, D., McAlister, H. A., Henry, T. J., et al. 2010, ApJS, 190, 1

Ramírez, I., Asplund, M., Baumann, P., Meléndez, J., \& Bensby, T. 2010, A\&A, 521,33

Reddy, B. E., Tomkin, J., Lambert, D. L., \& Allende Prieto, C. 2003, MNRAS, 340,304

Reglero, V., \& Fabregat, J. 1991, A\&AS, 90, 25

Ribas, I., Porto de Mello, G. F., Ferreira, L. D., et al. 2010, ApJ, 714, 384

Rocha-Pinto, H. J., Rangel, R. H. O., Porto de Mello, G. F., Bragança, G. A, \& Maciel, W. J. 2006, A\&A, 453, L9

Ryabchikova, T. A., Piskunov, N. E., Kupka, F., \& Weiss, W. W. 1997, BaltA, 6, 244

Santos, N. C., Bouchy, F., Mayor, M., et al. 2004, A\&A, 426, L19

Schuster, W. J., \& Nissen, P. E. 1988, A\&AS, 73, 225

Soderblom, D. R., \& Mayor, M. 1993, AJ, 105, 226

Spite, M. 1967, Ann. Astrophys., 30, 211

Steffen, M. 1985, A\&AS, 59, 403

Torres, C. A. O., Quast, G. R., da Silva, L., et al. 2006, A\&A, 460, 695

Twarog, B. A. 1980, ApJS, 44, 1

van Leeuwen, F. 2007, Hipparcos, the New Reduction of the Raw Data, Astrophys. Space Sci. Libr., 350

Warren, R. H., Jr., \& Hoffleit, D. 1987, BAAS, 19, 733

Whiting, E. E., \& Nicholls, R. W. 1974, ApJS, 27, 1

Yi, S., Demarque, P., Kim, Y.-C., et al. 2001, ApJS, 136, 417 